\title{
U.S. Hydropower Resource Assessment for Alaska
}

Prepared by: Alison M. Conner James E. Francfort

Project Manager:

Ben N. Rinehart

Published November 1997

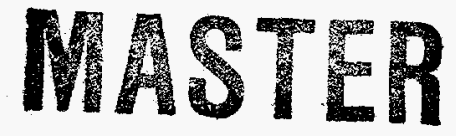

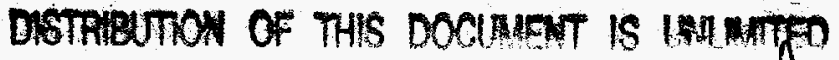

Idaho National Engineering and Environmental Laboratory

Renewable Energy Products Department

Lockheed Martin Idaho Technologies Company Idaho Falls, Idaho 83415

Prepared for the

U.S. Department of Energy

Assistant Secretary for Energy Efficiency and Renewable Energy

Under DOE Idaho Operations Office

Contract DE-AC07-94ID13223 


\section{DISCLAIMER}

This report was prepared as an account of work sponsored by an agency of the United States Government. Neither the United States Government nor any agency thereof, nor any of their employees, makes any warranty, express or implied, or assumes any legal liability or responsibility for the accuracy, completeness, or usefulness of any information, apparatus, product, or process disclosed, or represents that its use would not infringe privately owned rights. Reference herein to any specific commercial product, process, or service by trade name, trademark, manufacturer, or otherwise does not necessarily constitute or imply its endorsement, recommendation, or favoring by the United States Government or any agency thereof. The views and opinions of authors expressed herein do not necessarily state or reflect those of the United States Government or any agency thereof. 


\section{DISCLAIMER}

Portions of this document may be illegible electronic image products. Images are produced from the best available original document. 


\begin{abstract}
The U.S. Department of Energy is developing an estimate of the undeveloped hydropower potential in the United States. The Hydropower Evaluation Software (HES) is a computer model that was developed by the Idaho National Engineering Laboratory ${ }^{\mathrm{a}}$ for this purpose. HES measures the undeveloped hydropower resources available in the United States, using uniform criteria for measurement. The software was developed and tested using hydropower information and data provided by the Southwestern Power Administration. It is a menu-driven program that allows the personal computer user to assign environmental attributes to potential hydropower sites, calculate development suitability factors for each site based on the environmental attributes present, and generate reports based on these suitability factors. This report describes the resource assessment results for the State of Alaska.
\end{abstract}

a. In January 1997, the name of the Idaho National Engineering Laboratory (INEL) was changed to the Idaho National Engineering and Environmental Laboratory (INEEL). INEEL will be used throughout the text of the document, except where the use of INEL is historically important. 


\section{ACKNOWLEDGMENTS}

The authors thank Peggy A. M. Brookshier and John V. Flynn of the U.S. Department of Energy, and Roy Ireland of the State of Alaska for their active participation and timely comments. 


\section{CONTENTS}

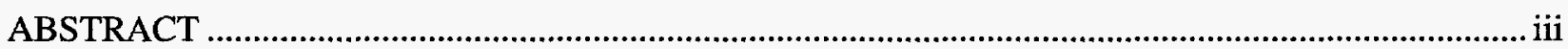

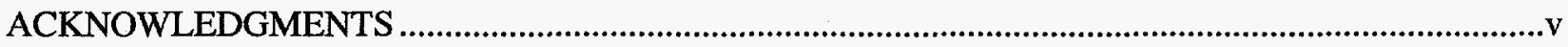

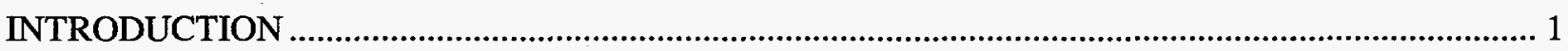

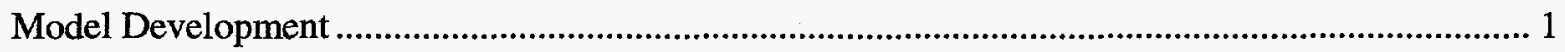

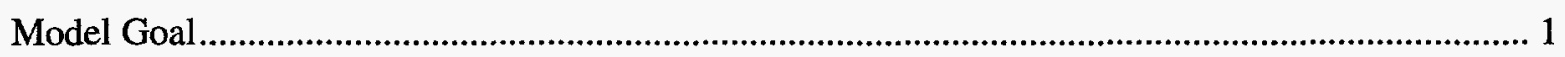

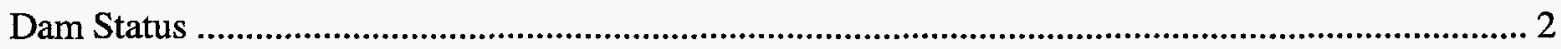

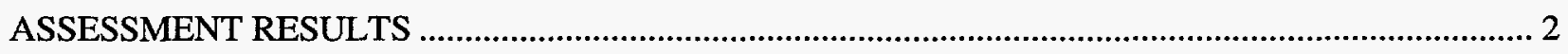

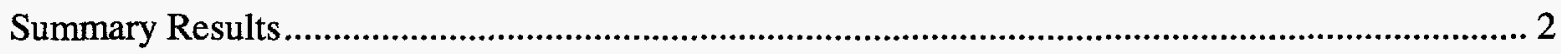

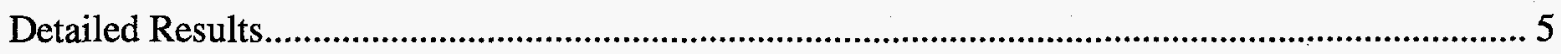

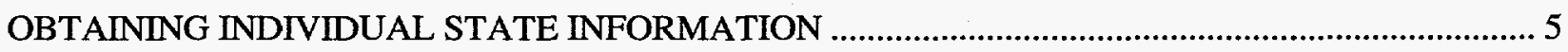

ADDITIONAL HYDROPOWER EVALUATION SOFTWARE INFORMATION ................................ 6

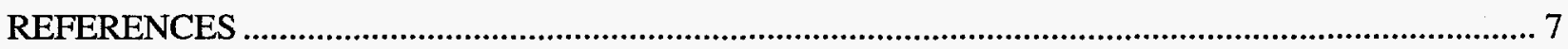

Appendix A-Summary Report

Appendix B-River Basins Report

Appendix C-Alaska Sites List

Appendix D-Individual Resource Database List

\section{FIGURES}

1. Number of sites, by capacity groups, with HES-modeled undeveloped hydropower potential.......... 3

2. The nonmodeled and HES-modeled undeveloped hydropower potential ....................................... 3

3. The number of sites with undeveloped hydropower potential and the total megawatts of HES-modeled undeveloped hydropower potential. 
4. Number of sites with undeveloped hydropower potential in the Alaska river basins

5. Megawatts of HES-modeled undeveloped hydropower potential in the Alaska river basins 5

\section{TABLES}

1. Undeveloped hydropower potential summary for Alaska ........................................................ 2 


\section{U.S. Hydropower Resource Assessment for Alaska}

\section{INTRODUCTION}

In June 1989, the U.S. Department of Energy initiated the development of a National Energy Strategy to identify the energy resources available to support the expanding demand for energy in the United States. Public hearings conducted as part of the strategy development process indicated that undeveloped hydropower resources were not well defined. As a result, the Department of Energy established an interagency Hydropower Reșource Assessment Team to ascertain the undeveloped hydropower potential. In connection with these efforts by the Department of Energy, the Idaho National Engineering Laboratory designed the Hydropower Evaluation Software (HES), which has been used to perform a resource assessment of the undeveloped conventional hydropower potential in over 30 states. This report presents the results of the hydropower resource assessment for the State of Alaska. Undeveloped pumped storage hydropower potential is not included.

The HES was developed as a tool to measure undeveloped hydropower potential regionally or by state. The software is not intended to provide precise development factors for individual sites, but to provide regional or state totals. Because the software was developed as a generic measurement tool encompassing national issues, regional and state totals must be considered judiciously; various local issues may skew undeveloped hydropower potential totals. The information for the resource assessment was compiled from the Federal Energy Regulatory Commission's Hydroelectric Power Resources Assessment database and several other sources. Refer to DOE/ID-10338, the User's Manual (Francfort, Matthews, Rinehart 1991) for the specifics of the software and to DOE/ID-10430.1, the Status Report (Conner, Francfort, Rinehart 1996) for an overview of all resource assessment activities to date.

\section{Model Development}

Hydropower Evaluation Software, both a probability-factor computer model and a database, is a menu-driven program that is intended to be user-friendly. Computer screens and report-generation capabilities were developed to meet the needs of users nationwide. The software uses environmental attribute data to generate an overall project environmental suitability factor (PESF) between 0.1 and 0.9 , where 0.9 indicates the highest likelihood of development and 0.1 indicates the lowest likelihood of development. The suitability factors depend on the unique environmental attributes of each potential site. They reflect the considerations that (a) environmental concerns can make a potential site unacceptable, prohibiting its development (for a suitability factor of 0.1 ), or (b) if there are no environmental concerns, there is no negative effect on the likelihood of site development (for a suitability factor of 0.9). A combination of attributes can result in a lower suitability factor because multiple environmental considerations would reduce the likelihood that a site may be developed to its physical potential.

\section{Model Goal}

The goal of the HES is to assemble an accurate resource database of all sites with undeveloped hydropower potential in the United States for use as a planning tool to determine the viable national hydropower potential. Undeveloped hydropower potential is not limited to the development of new sites; it also includes the development of additional hydropowergenerating capacity at sites that currently have hydropower, but are not developed to their full potential. This undeveloped hydropower potential is a source of nonpolluting, renewable energy available to meet the growing power needs of the United States. The HES should help make this goal obtainable and ensure a set of uniform criteria for national assessment. 


\section{Dam Status}

The effects of environmental attributes vary by dam status. The dam status classifications used are as follows:

$$
\begin{aligned}
& \text { W = Developed hydropower site } \\
& \text { with current power generation, } \\
& \text { but the total hydropower po- } \\
& \text { tential has not been fully de- } \\
& \text { veloped. Only the undeveloped } \\
& \text { hydropower potential is dis- } \\
& \text { cussed in this report. }
\end{aligned}
$$

$\mathrm{W} / \mathrm{O}=$ Developed site without current power generation. The site has some type of developed impoundment or diversion structure, but no developed hydropower generating capability.

$\mathrm{U}=$ Undeveloped site. The site does not have power generation capability nor a developed impoundment or diversion structure.

\section{ASSESSMENT RESULTS Summary Results}

A total of 119 sites (Table 1) have been identified and assessed for their undeveloped hydropower potential. The HES results for individual site capacities range from 6 kilowatts
(kW) to 510 megawatts (MW). The majority (79\%) of the sites in Alaska are less than 10 MW (Figure 1).

The nonmodeled undeveloped hydropower potential total for Alaska was identified as 4,042 MW. The HES results lowers this estimate about $47 \%$ to $2,157 \mathrm{MW}$. The greatest reduction in undeveloped hydropower potential, by $\mathrm{MW}$, occurs at sites with some type of developed impoundment or diversion structure, but no current power generation capability (W/O category). These sites have a HES-modeled undeveloped hydropower potential of 1,610 $\mathrm{MW}$, a 1,256-MW reduction in the estimated undeveloped hydropower potential (Figure 2). Figure 3 correlates the number of sites that have undeveloped hydropower potential with the total megawatts of HES-modeled undeveloped hydropower potential. The developed sites without power have the largest average undeveloped hydropower potential of about $27 \mathrm{MW}$ per site (Figure 3).

The 119 identified sites are located within 10 major river basins. The number of sites per major river basin ranges from 2 in the Copper River Basin to 37 in the Juneau Group River Basin (Figure 4). Fifty-three percent of the undeveloped hydropower potential in the State of Alaska is contained within the Susitna River Basin (Figure 5).

Table 1. Undeveloped hydropower potential summary for Alaska. The table contains the nonmodeled undeveloped nameplate potential and the HES-modeled undeveloped potential totals.

\begin{tabular}{lccc} 
& Number of projects & Nameplate potential & HES-modeled potential \\
& & $(\mathrm{MW})$ & $(\mathrm{MW})$ \\
\cline { 2 - 4 } With Power & 3 & 64.7 & 58.0 \\
W/O Power & 60 & $2,866.2$ & $1,609.9$ \\
Undeveloped & 56 & $1,111.4$ & 489.5 \\
State Total & 119 & $4,042.3$ & $2,157.4$ \\
\hline
\end{tabular}




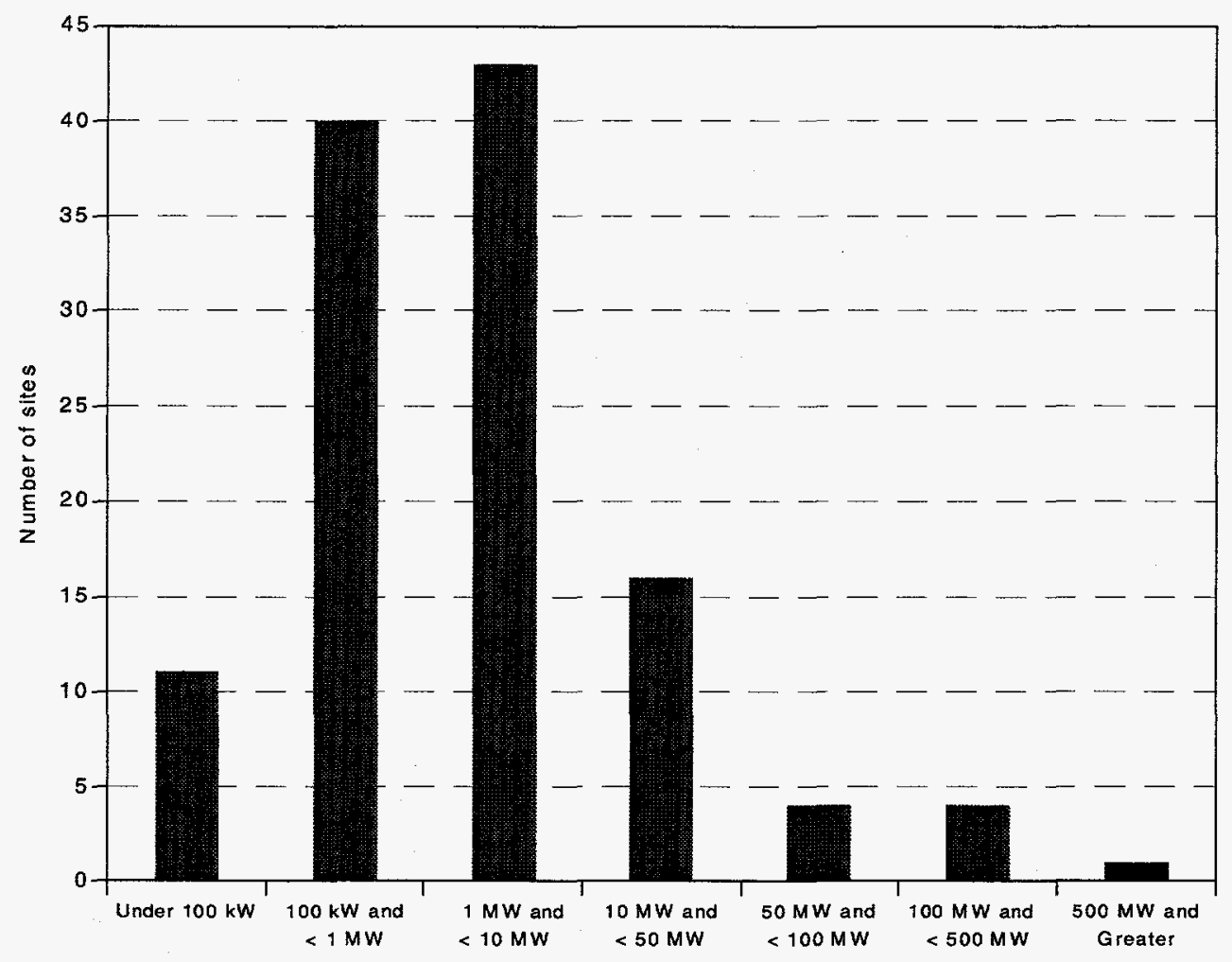

Figure 1. Number of sites, by capacity groups, with HES-modeled undeveloped hydropower potential.

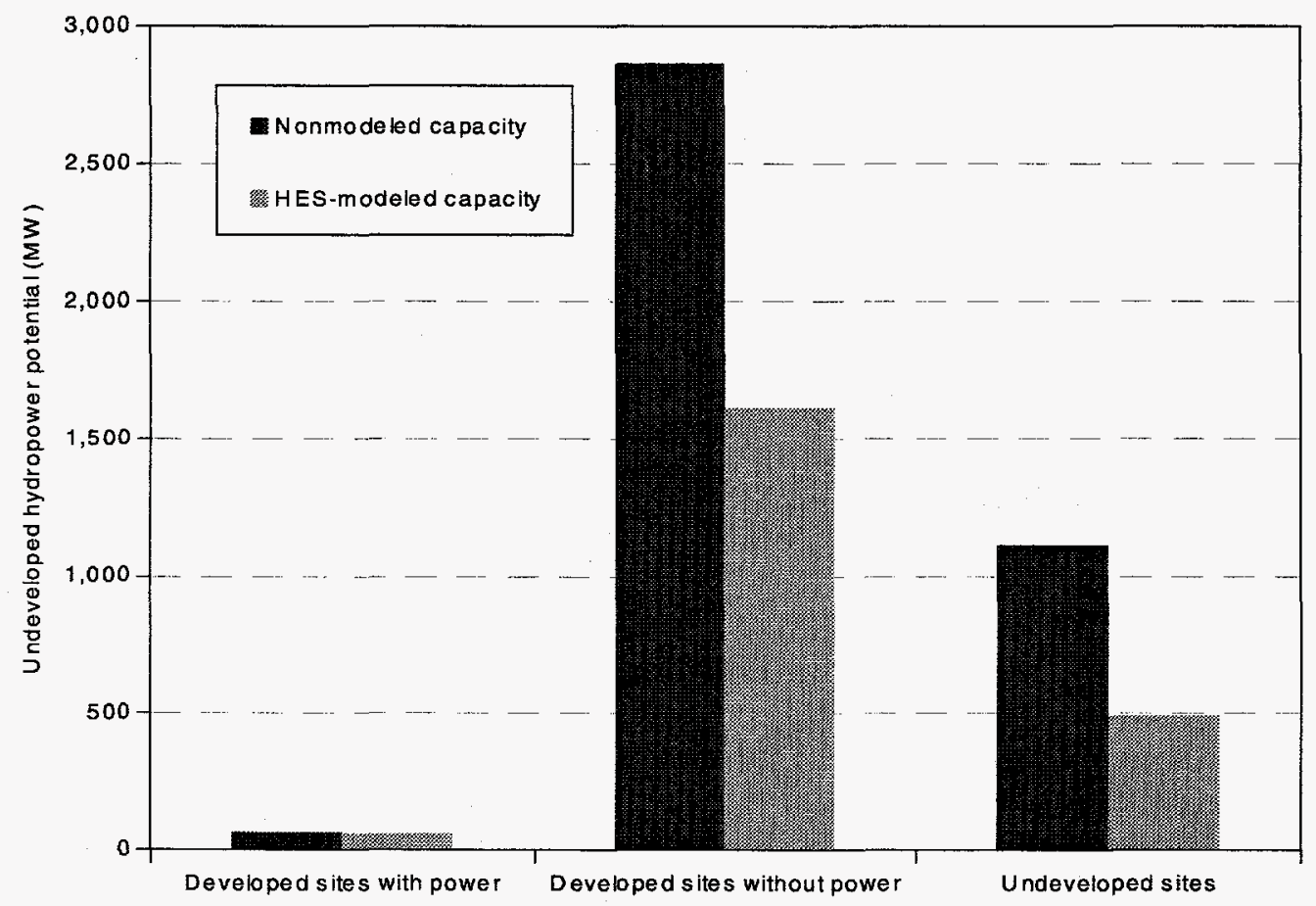

Figure 2. The nonmodeled and HES-modeled undeveloped hydropower potential. 


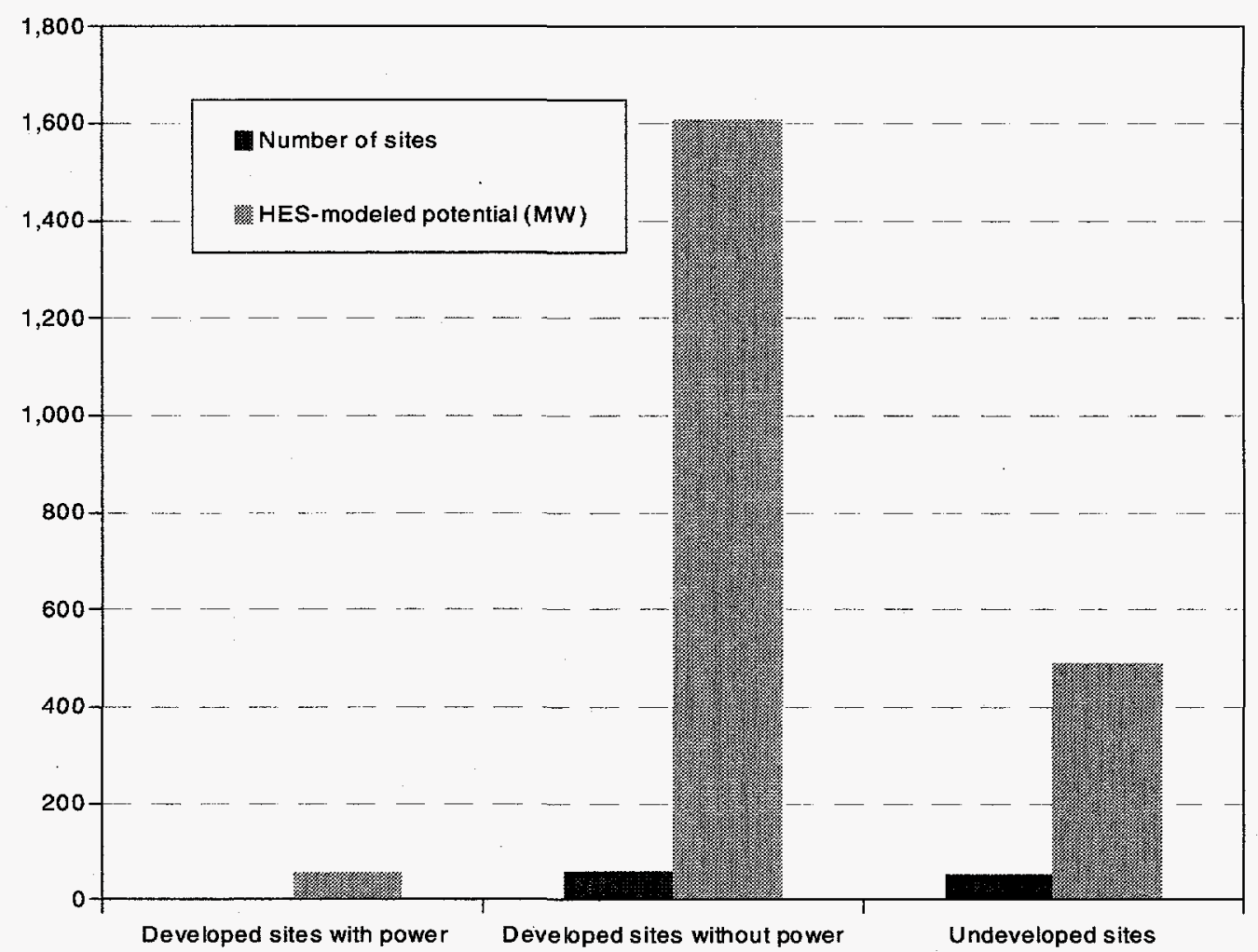

Figure 3. The number of sites with undeveloped hydropower potential and the total megawatts of HES-modeled undeveloped hydropower potential.

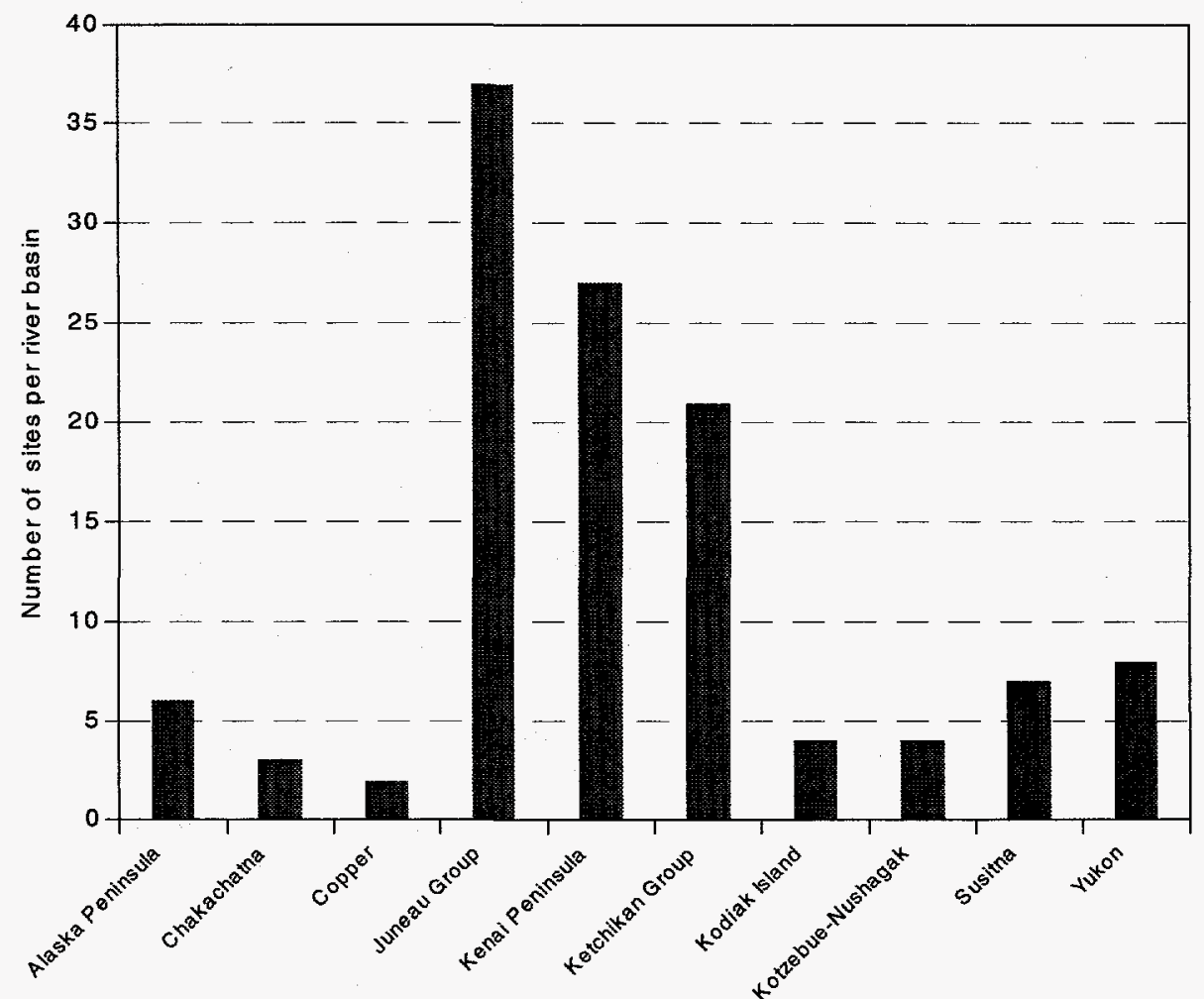

Figure 4. Number of sites with undeveloped hydropower potential in the Alaska river basins. 


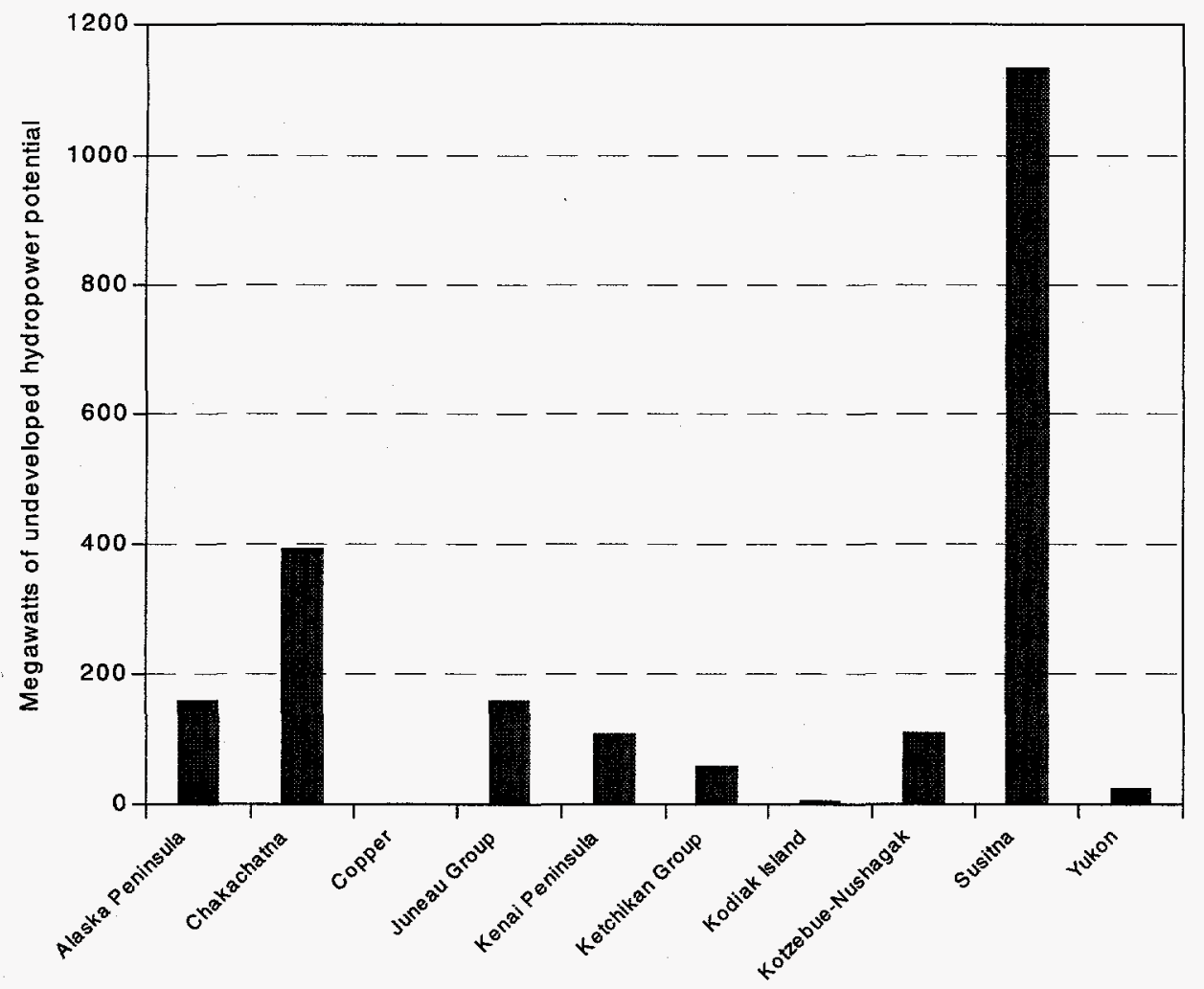

Figure 5. Megawatts of HES-modeled undeveloped hydropower potential in the Alaska river basins.

\section{Detailed Results}

The appendices contain, in the form of HES-generated reports, detailed information about the undeveloped hydropower potential in Alaska. The appendices contain the following information:

Appendix A summarizes the undeveloped hydropower potential by dam status groups. The number of sites, nonmodeled undeveloped hydropower potential, and HES-modeled undeveloped hydropower potential is provided based on the dam status.

Appendix B provides the hydropower resource assessment by river basin, which includes the project number, project name, stream name, dam status, nonmodeled undeveloped hydropower potential, and the HES-modeled undeveloped hydropower potential for each site. Subtotals are provided for each river basin.

Appendix $\mathbf{C}$ lists the project numbers, plant name, stream name, if a site is Federally owned, nonmodeled undeveloped hydropower potential, and HES-modeled undeveloped hydropower potential. The sites are grouped by dam status.

Appendix D contains a resource database list for the 119 sites in Alaska. Information includes plant name, stream, state, county, river basin and owner names, project number, nameplate and HES-modeled undeveloped hydropower potential, the unit and plant types, dam status, latitude, longitude, and the environmental factors that the HES uses to determine the PESF.

\section{OBTAINING INDIVIDUAL STATE INFORMATION}

Additional copies of the hydropower resource assessment results for individual states are available and can be obtained by writing or calling the authors or the National Technical Information Service (NTIS).

Telephone Orders-(703) 487-4650. NTIS sales desk and customer services are available 
between 8:30 a.m. and 5:00 p.m., Eastern Standard Time.

Fax-(703) 321-8547. Customers may fax their orders to NTIS. These orders may be charged to a NTIS deposit account, American Express, VISA, or MasterCard.

Mail Orders-Mail orders should be sent to National Technical Information Service, Document Sales, 5285 Port Royal Road, Springfield, VA 22161. Call the sales desk for prices before placing an order.

E-mail-Customers may e-mail their requests to info@ntis.fedworld.gov.

Method of Payment-Customers may pay for reports (and other NTIS products and services) by (a) credit card (American Express, Visa or MasterCard); (b) check or money order on a United States bank payable to NTIS; (c) NTIS deposit account; or, (d) by asking to be billed (add $\$ 7.50$ per order), United States, Canada, and Mexico, only.

Handling Fee-A $\$ 4.00$ handling fee per total order applies to orders from the United States, Canada, and Mexico. Handling charges do not apply to rush order service or pick-up orders.

Postage and Shipping-Orders are shipped first class mail, or equivalent, to addresses in the United States, Canada, and Mexico.

Order Turnaround Time-Orders for technical reports generally are shipped within 3 to 5 days of receipt. For faster service, NTIS offers rush order service.

Rush Order Service-Call 1-800-533-NTIS. In Virginia, Canada, and Mexico call (703) $487-4700$. For NTIS rush order service add $\$ 15$ per item. This guarantees that an order will be processed through NTIS within 24 hours of its receipt. These orders receive immediate, individual attention. The items ordered are delivered by first call mail. Call NTIS for information on rush order service for computer products.

For Help in Tracing an Order-Call (703) $487-4650$ and request the customer service option.

\section{ADDITIONAL HYDROPOWER EVALUATION SOFTWARE INFORMATION}

Additional information concerning the HES can be obtained by contacting Ben Rinehart or Jim Francfort at the addresses provided below. Copies of the software and the User's Manual may also be obtained from these individuals.

Ben Rinehart, Project Manager

Idaho National Engineering and Environmental Laboratory

P.O. Box 1625, MS 3830

Idaho Falls, ID 83415-3830

(208) 526-1002

Jim Francfort

Idaho National Engineering and Environmental Laboratory

P.O. Box 1625, MS 3830

Idaho Falls, ID $83415-3830$

(208) 526-6787

Information concerning the State of Alaska's involvement with the resource assessment or about the identified sites may be obtained by contacting:

Roy Ireland

State of Alaska

Department of Natural Resources

Division of Mining and Water Management

3601 C Street, Suite 800

Anchorage, AK 99503

(907) 269-8639 


\section{REFERENCES}

Conner, A. M., J. E. Francfort, and B. N. Rinehart, 1996, Uniform Criteria for U.S. Hydropower Resource Assessment, Hydropower Evaluation Software Status Report-II, DOE/ID 10430.1, Idaho National Engineering Laboratory, Idaho Falls, Idaho.

Francfort, J. E., S. D. Matthews, and B. N. Rinehart, 1991, Hydropower Evaluation Software User's Manual, DOE/ID-10338, Idaho National Engineering Laboratory, Idaho Falls, Idaho. 
Appendix A

Summary Report 
DATE: $10 / 31 / 97$

H Y D R O P O W E R C A P A C I T Y S U M M A R Y

\begin{tabular}{|c|c|c|c|c|}
\hline state & Category & $\begin{array}{l}\text { Number of } \\
\text { Projects }\end{array}$ & $\begin{array}{l}\text { Name Plate } \\
\text { Capacity }(\mathrm{KW})\end{array}$ & $\begin{array}{l}\text { HES Adjusted } \\
\text { Capacity (KW) }\end{array}$ \\
\hline \multirow[t]{4}{*}{$\mathrm{AK}$} & with Power & 3 & 64650 & 58010 \\
\hline & W/O Power & 60 & 2866227 & 1609854 \\
\hline & Undeveloped & 56 & 1111380 & 489546.50 \\
\hline & STATE TOTAL & 119 & 4042257 & 2157410.50 \\
\hline \multirow[t]{3}{*}{ TOTALS } & With Power & 3 & 64650 & 58010 \\
\hline & w/O Power & 60 & 2866227 & 1609854 \\
\hline & Undeveloped & 56 & 1111380 & 489546.50 \\
\hline \multicolumn{2}{|c|}{ GRAND TOTAL } & 119 & 4042257 & 2157410.50 \\
\hline
\end{tabular}




\section{Appendix B}

River Basins Report 
Page No.

$10 / 31 / 97$

FERC

Number

HYDROPOWER RESOURCE ASSESSMENT BY RIVER BASIN

Dam Name Plate

stat Rating (KW) PESF PESF * KW
** River Basin ALASKA PENINSULA RIVER BAS

AK010 TAZIMINA

" TAZIMINA R $\begin{array}{ll}11316 & \text { TAZIMINA RIVER } \\ & \text { TAZIMINA } \mathrm{R}\end{array}$

AK007 AKUTAN UNNN STR

AK008

INDIAN CREEK

INDIAN CR, CHIGNIK BAY

AKO09 ILIAMNA

KVICHAK R

AK060 PYRAMID CREEK

PYRAMID CR, PACIFIC OCEAN

** Subtotal **

** River Basin CHAKACHATNA RIVER BASINS

AK012 CHAKACHAMNA

CHAKACHAMNA $\mathrm{R}$

AK013 COFFEE

BELUGA R

AK014 UPPER BELUGA

BELUGA $R$

** Subtotal **

** River Basin COPPER RIVER BASIN

10686 LAKE REDFIELD

LAKE REDFIELD, YAKUTAT BAY

08767 BEARTRAIL

BEARTRAII CR, IBECK CR

** Subtotal **

** River Basin JUNEAU GROUP

11077 GOAT LAKE

SKAGWAY R(GOAT LAKE)
U

U

wo

wo

wo

wo

$\mathrm{U}$

Wo

wo

334370.00

$366000.00 \quad 0.90$

$37000.00 \quad 0.75$

$48000.00 \quad 0.75$

451000.00

U

wo

$\mathrm{U}$

$$
700.00 \quad 0.10
$$

$170.00 \quad 0.90$

$1100.00 \quad 0.10$

$313000.00 \quad 0.50$

$1400.00 \quad 0.90$

$2000.00 \quad 0.50$

2850.00

$6000.00 \quad 0.50$

$$
18000.00 \quad 0.10
$$

1260.00

159893.00

329400.00

27750.00

36000.00

393150.00

1000.00

425.00

1425.00

3000.00 
Page No.

$10 / 31 / 97$

FERC

Number

HYDROPOWER RESOURCE ASSESSMENT BY RIVER BASIN

Plant Name/

stream
Dam Name Plate

stat Rating ( $K W$ ) PESF

CARBON LAAKE

CARBON LAKE

AKO28 MILK IAKE

MILK CR

AK029 BORODINO LAKE

BIG PORT WALTER, CHATHAM STRAIT

AK030 BETTY LAKE

PORT ARMSTRONG, CHATHAM STRAIT

AK032 DIANA LAKE

UNN STR

AKO 34 PELICAN

PELICAN CR,LISIANSKI INLET

AK035 GOLD CREEK

GOLD CR

AK037 LAKE DOROTHY

DOROTHY CR

AK038 SPEEL DIVISION

SPEEL $R$

AK039 CRATER LAKE

CRATER CR, SPEEL $R$

AK042 UPPER SWEETHEART

SWEETHEART FALIS CR

02755A THOMAS BAY

THOMAS BAY

AKO 43 SCENERY

SCENERY CR

06856 THOMAS BAY

CASCADE CR

06211 THOMAS BAY

CASCADE CR
$\mathbf{U}$

U

$7000.00 \quad 0.50$

$10000.00 \quad 0.25$

$7000.00 \quad 0.25$

1750.00

U

$5000.00 \quad 0.25$

1250.00

U

$$
200.00 \quad 0.25
$$

50.00

U

$8000.00 \quad 0.25$

2000.00

U

$1000.00 \quad 0.25$

U

$2000.00 \quad 0.90$

$\mathrm{U}$

$34000.00 \quad 0.50$

$\mathrm{U}$

$63000.00 \quad 0.25$

15750.00

$\mathrm{U}$

$27000.00 \quad 0.25$

6750.00

U

$7000.00 \quad 0.50$

$\mathrm{U}$

$30000.00 \quad 0.25$

$\mathrm{U}$

$15000.00 \quad 0.10$

250.00

1800.00

17000.00

3500.00

7500.00

1500.00

$\mathrm{U}$

$4400.00 \quad 0.10$

440.00

$\mathrm{U}$

$34000.00 \quad 0.10$

3400.00 
Page No.

$10 / 31 / 97$

FERC

Number

02755B

FALIS LAKE

CASCADE CR, THOMAS BAY

AK044 RUTH LAKE

DELTA CR

11319 HAINES

UNN STREAM, CHILKOOT R

08769 KASIDAYA CREEK

KASIDAYA CREEK, TAIYA INLET

11290 TAIYA INLET

DAYEBAS CR, KATZEHIN $R$

$\begin{array}{ll}\text { AK025 TAKATZ } & \\ & \text { TAKATZ CR, TAKATZ BAY }\end{array}$

AK031 MAKSOUTOF

MAKSOUTOF $\mathrm{R}$

02267 PULP MILL

MEDVETCHA $R$

AK033 SUPERIOR
ELFIN COVE (WACHUSETT COVE CR)

09763 NUGGET CREEK

NUGGET CR, MENDENHAL IK

04641 DANNER-FENSTER

UNN STR, GASTINEAU CHANNEL

09760 TREADWELL DITCH

TREADWELL DH, GASTINEAU CHANNEL

10681 SHEEP CREEK

SHEEP CR, TAKU INLET

AK036 ANNEX CREEK

ANNEX CR, TAKU INLET

AK040 TEASE

LONG $\mathrm{R}$

AK041 SWEETHEART FALLS

SWEETHEART FALIS CR
Dam Name Plate

stat Rating (KW) PESF PESF * KW

$\mathrm{U}$

$8000.00 \quad 0.10$

800.00

$\mathrm{U}$

$13000.00 \quad 0.50$

6500.00

$\mathrm{U}$

$2500.00 \quad 0.25$

625.00

Wo

$700.00 \quad 0.10$

70.00

wo

$2000.00 \quad 0.75$

1500.00

WO

$20000.00 \quad 0.75$

15000.00

wo

$24000.00 \quad 0.50$

12000.00

wo

$$
900.00 \quad 0.50
$$

450.00

wo

$$
750.00 \quad 0.75
$$

562.50

wo

$2200.00 \quad 0.50$

1100.00

wo

$12.00 \quad 0.50$

6.00

wo

$2200.00 \quad 0.90$

1980.00

wo

$3100.00 \quad 0.50$

1550.00

Wo

$1750.00 \quad 0.50$

875.00

wo

$16000.00 \quad 0.75$

12000.00

wo

$29000.00 \quad 0.50$

14500.00 
Page No.

$10 / 31 / 97$

FERC

Number

HYDROPOWER RESOURCE ASSESSMENT BY RIVER BASIN

Plant Name/
Stream
Dam Name Plate

stat Rating (KW) PESF PESF * $\mathrm{KW}$

$\begin{array}{ll}\text { AK045 } & \begin{array}{l}\text { GOAT } \\ \text { GOAT CR }\end{array} \\ \text { AK046 } & \begin{array}{l}\text { BLIND SLOUGH } \\ \text { CRYSTAL CR, BLIND R }\end{array} \\ 10566 & \begin{array}{l}\text { LUTAK INLET } \\ \text { UNN STR, LUTAK INLET }\end{array} \\ 11287 & \begin{array}{l}\text { LACE RIVER } \\ \text { UNN STR, EVELYN LAKE }\end{array} \\ * * \text { SubtotaI ** }\end{array}$

WO

$20000.00 \quad 0.75$

15000.00

Wo

Wo

Wo

$1400.00 \quad 0.50$

700.00

112.50

$150.00 \quad 0.75$

$4900.00 \quad 0.75$

3675.00

413162.00

160946.00

** River Basin KENAI PENINSULA RIVER BAS

08827

SHIP CREEK

CITY WATER \& WASTE WTR PIPELIN

$$
510.00 \quad 0.90
$$

459.00

05216 ALYESKA CREEK

$\mathrm{U}$

ALYESKA CR, GLACIER CR

$1500.00 \quad 0.25$

375.00

$\mathrm{U}$

$830.00 \quad 0.25$

207.50

GRANT CR, TRAIL $R$

07633 GRANT LAKE

UP TRAIL LK(GRANT LK), TRAIL $R$

AK02 4 MAIN LAKE

MAIN BAY, PRINCE WILLIAM SOUND

$\mathrm{U}$

$\mathrm{U}$

$7000.00 \quad 0.25$

1750.00

$$
125.00 \quad 0.25
$$

31.25

$\mathrm{U}$

ALLISON CR

$8000.00 \quad 0.25$

2000.00

U

$15000.00 \quad 0.25$

3750.00

DUCK R, GALENA BAY

$\mathrm{U}$

P C 1

POWER CR, EYAK LAKE

$\mathrm{U}$

08770 P C 2
POWER CR, EYAK LAKE

10064 WHITTIER CREEK

WHITTIER CR, PASSAGE CANAL

U

$5000.00 \quad 0.10$

500.00

$2000.00 \quad 0.10$

200.00

$200.00 \quad 0.25$

50.00

U

$6000.00 \quad 0.10$ 
Page No.

10/31/97

FERC

Number

$10685 \mathrm{~A}$

CRATER LAKE

CRATER IAAKE,ORCA INLET

AK062 PALMER

MCROBERTS CREEK

AK022 BRADLEY LAKE

BRADLEY CR

AK020 SNOW

SNOW R

08756 JUNEAU CREEK
JUNEAU CR, KENAI R

AK021 SELDOVIA

FISH CR

AK023 LOWELL

LOWELI CR, DAY HARBOR

08768 LOST CREEK

LOST CR, SALMON CR, RESURRECTION

08765 BEAR CREEK

BEAR CR, TURNAGAIN ARM

06601 CALIFORNIA CREEK

CALIFORNIA CR, PLACER R

02251 SAN JUAN

SAN JUAN LAKE

11417 SNYDER FALLS CREEK

SNYDER FALLS CR,NELSON BAY

10685B CRATER LAKE

CRATER LAKE, ORCA INLET

08766 STEEP CREEK

STEEP CR, EYAK R

AK061 SOUTH FORK EAGLE RIVER

$S$ FK EAGLE $R$

11243 POWER CREEK

POWER CR, EYAK LAKE
Dam Name Plate

stat Rating (KW) PESF PESF * KW

U

$600.00 \quad 0.25$

150.00

$\mathrm{U}$

$400.00 \quad 0.25$

100.00

W

$63900.00 \quad 0.90$

57510.00

WO

$63000.00 \quad 0.50$

31500.00

wo

$1800.00 \quad 0.50$

900.00

wo

$460.00 \quad 0.50$

230.00

wo

$650.00 \cdot 0.75$

487.50

wo

$900.00 \quad 0.75$

675.00

wo

$250.00 \quad 0.50$

125.00

wo

$1500.00 \quad 0.75$

1125.00

wo

$100.00 \quad 0.75$

75.00

wo

$1450.00 \quad 0.75$

1087.50

wo

$1000.00 \quad 0.75$

750.00

wo

$870.00 \quad 0.75$

652.50

wo

$1100.00 \quad 0.75$

825.00

wo

$5000.00 \quad 0.50$

2500.00 
HYDROPOWER RESOURCE ASSESSMENT BY RIVER BASIN

$\begin{array}{cc}\text { FERC } & \text { Plant Name/ } \\ \text { Number } & \text { Stream }\end{array}$

** Subtotal **

** River Basin KETCHIKAN GROUP

AKO49 HARDING

HARDING $R$

05215 FISH CREEK

FISH CR, SALMON R

05214 WEST MT HINCHLEY

$W$ HINCKLEY RUN, SALMON R

$\begin{array}{ll}05212 & \text { THUMB CREEK } \\ & \text { THUMB CR, SALMON R }\end{array}$

11393 MOHONEY LAKE

MAHONEY CR

AK055 LAKE CONNELL

WARD CR, WARD COVE

AK057 LAKE MARY

OLD FRANKS CR

AK058 BLACK BEAR

BLACK BEAR CR

09996 REDISCOVERY LODGE

BELL CR

11508 WOLF LAKE

WOLF CR

AK051 PURPLE LAKE

PURPLE LAKE OUTLET

10773 BURNETT R HATCHERY

BURNETT $R$

AK047 ANITA IAKE

ANITA CR, KUNK CR

AK048 VIRGINIA LAKE

MILL CR

AK050 LAKE GRACE

GRACE CR, BEHM CANAL
Dam Name Plate

stat Rating (KW) PESF PESF * KW
108615.25

$\mathrm{U}$

U

$\mathrm{U}$

U

U

U

U

$\mathrm{U}$

$\mathrm{U}$

U

W

W

wo

พo

wo

$$
189145.00
$$

$$
18000.00 \quad 0.10
$$$$
1300.00 \quad 0.25
$$$$
2600.00 \quad 0.25
$$$$
10000.00 \quad 0.25
$$$$
9000.00 \quad 0.25
$$

$2000.00 \quad 0.10$

$9600.00 \quad 0.25$

$5000.00 \quad 0.25$

$115.00 \quad 0.25$

$2500.00 \quad 0.25$

$$
500.00 \quad 0.75
$$

$250.00 \quad 0.50$

$3230.00 \quad 0.50$

$12000.00 \quad 0.50$

$21000.00 \quad 0.50$
1800.00

325.00

650.00

2500.00

2250.00

200.00

2400.00

1250.00

28.75

625.00

375.00

125.00

1615.00

6000.00

10500.00 
Page No.

$10 / 31 / 97$

FERC

Number

AK052

MAHONEY LAKE

MAHONEY CR

AK053 UPPER SILVIS

BEAVER FALLS CR, GEORGE INLET

02328

LAKE WHITMAN

CASE CR

AK054 KETCHIKAN LAKES

KETCHIKAN CR

AK056 IINKUM

LINKUM CR, KASAAN BAY

11480 UPPER REYNOLDS CREEK

REYNOLDS CR, COPPER HARBOR

** Subtotal **
HYDROPOWER RESOURCE ASSESSMENT BY RIVER BASIN

Dam Name Plate

stat Rating (KW) PESF PESF * KW

wo

WO

WO

Wo

wo

wo

$14400.00 \quad 0.75$

10800.00

10000.00

$20000.00 \quad 0.50$

$2500.00 \quad 0.75$

1875.00

$2000.00 \quad 0.50$

1000.00

$4560.00 \quad 0.75$

3420.00

1000.00

$2000.00 \quad 0.50$

142555.00

58738.75

$$
200.00 \quad 0.25
$$

50.00

MENNONITE CR, SETTLERS COVE

$\mathrm{U}$

11497

LEANNE IAAKE

LEANNE LAKE OUTLET

$2800.00 \quad 0.90$

wo

01432

DRY SPRUCE

UNN TRIB, DRY SPRUCE BAY

$$
75.00 \quad 0.50
$$

พO

11139

TERROR LAKE

TERROR $\mathbf{R}$

$$
3000.00 \quad 0.75
$$

2250.00

** Subtotal **

$$
6075.00
$$

4857.50

GRANT LAKE
WOOD $R$, KOKWOK $R$

U

AKO Riv

Basin KOTZEBUE-NUSHAGAK RIVER B

03175 KISARALIK Wo

KISARALIK $\mathbf{R}$

AK004 LAKE ELVA

ELVA CR

wo

Wo

AK006

NUYAKUK (TIKCHIK)

NUYAKUK $R$
$2700.00 \quad 0.25$

675.00

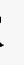

$30000.00 \quad 0.50$

15000.00

$1500.00 \quad 0.75$

1125.00

$127000.00 \quad 0.75$

95250.00 


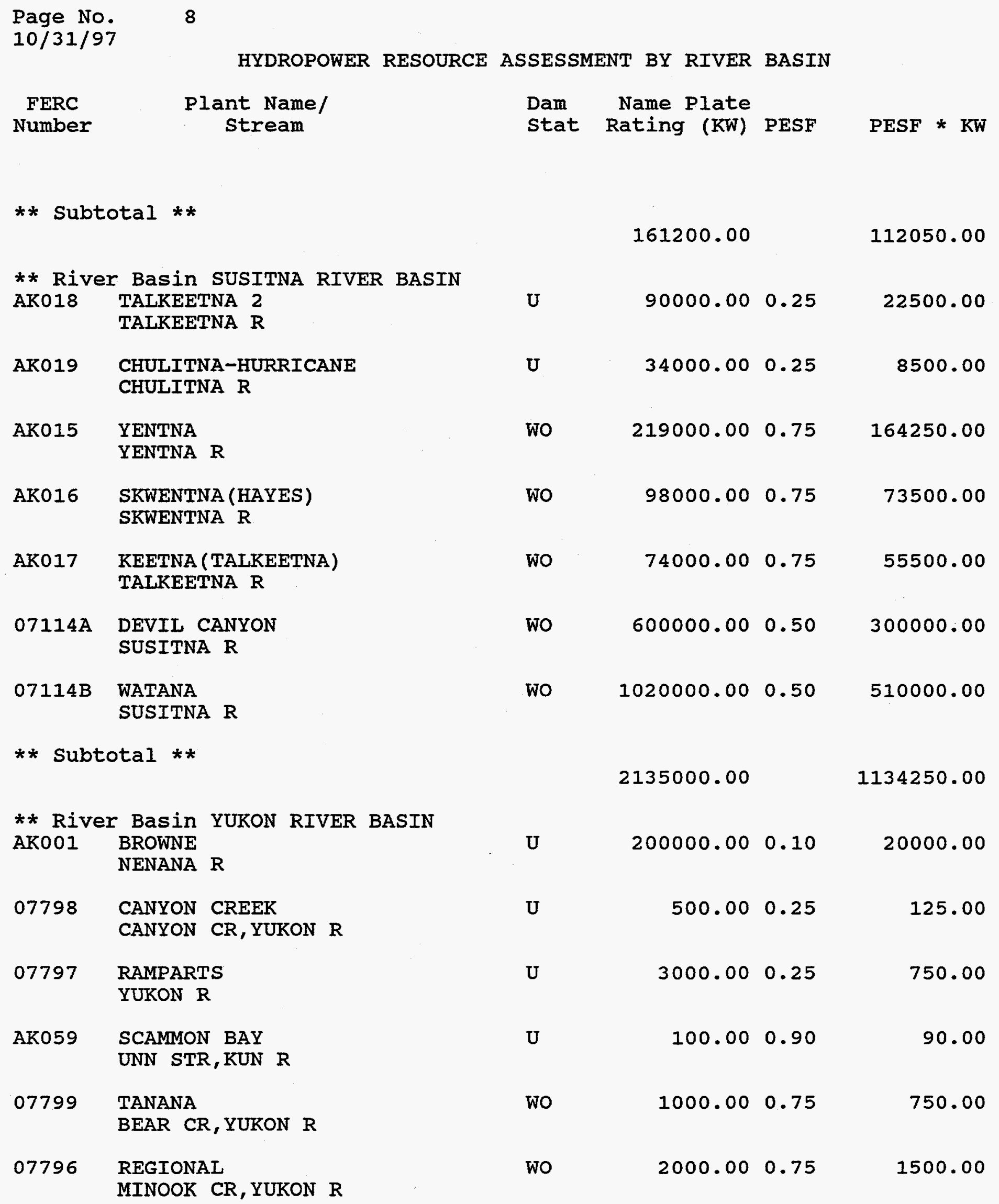


Page No.

$10 / 31 / 97$

FERC

Number

Plant Name/

stream

AK002

SCAMMON

UNN STR, SCAMMON BAY

AK003 SCAMMON (PHASE 2)

UNN STR, SCAMMON BAY
Dam Name plate

Stat Rating (KW) PESF PESF * KW

wo

$175.00 \quad 0.90$

พั

$125.00 \quad 0.90$

112.50

** Subtotal **

*** Total ***
206900.00

4042257.00
23485.00

2157410.50 
Appendix C

Alaska Sites List 
HYDROPOWER RESOURCE ASSESSMENT BY FERC NUMBER

Plant Name/

stream

** FERC Number 02755A

THOMAS BAY

THOMAS BAY

** Subtotal **

** FERC Number 02755B

FALLS LAKE

CASCADE CR, THOMAS BAY

** Subtotal **

** FERC Number 02760

P C 1

POWER CR, EYAK LAKE

** Subtotal **

** FERC Number 05212

THUMB CREEK

THUMB CR, SALMON $R$

** Subtotal **

** FERC Number 05214

WEST MT HINCHLEY

W HINCKLEY RUN, SALMON $R$

** Subtotal **

** FERC Number 05215

FISH CREEK

FISH CR, SALMON R

** Subtotal **

** FERC Number 05216

ALYESKA CREEK

ALYESKA CR, GLACIER CR
Dam Name Plate

ST Stat Rating (KW) PESF

PESF * KW

AK U

$30000.00 \quad 0.25$

7500.00

30000.00

7500.00

AK U

$8000.00 \quad 0.10$

800.00

$$
8000.00
$$

800.00

AK U

$5000.00 \quad 0.10$

500.00

5000.00

500.00

AK U

$10000.00 \quad 0.25$

2500.00

10000.00

2500.00

AK U

$2600.00 \quad 0.25$

650.00

2600.00

650.00

AK U

$1300.00 \quad 0.25$

325.00

1300.00

325.00

AK U

$1500.00 \quad 0.25$

375.00 
HYDROPOWER RESOURCE ASSESSMENT BY FERC NUMBER

Plant Name/
stream

** Subtotal **

** FERC Number 06211

THOMAS BAY

CASCADE CR

** Subtotal **

** FERC Number 06856

THOMAS BAY

CASCADE CR

** Subtotal **

* * FERC Number 07633

GRANT LAKE

UP TRAIL LK(GRANT LK), TRAII R

** Subtotal **

** FERC Number 07797

RAMPARTS

YUKON R

** Subtotal **

** FERC Number 07798

CANYON CREEK

CANYON CR, YUKON $R$

** Subtotal **

** FERC Number 08770

$\mathrm{P} C 2$

POWER CR, EYAK LAKE

** Subtotal ** $\begin{array}{ll}\text { Dam } & \text { Name Plate } \\ \text { ST } & \text { Stat } \\ \text { Rating (KW) PESF }\end{array}$

$$
1500.00
$$

375.00

AK U

$34000.00 \quad 0.10$

3400.00

34000.00

3400.00

AK U

$4400.00 \quad 0.10$

440.00

4400.00

440.00

AK U

$7000.00 \quad 0.25$

1750.00

7000.00

1750.00

AK U

$3000.00 \quad 0.25$

750.00

3000.00

750.00

AK U

$500.00 \quad 0.25$

125.00

500.00

125.00

AK U

$2000.00 \quad 0.10$

200.00

2000.00

200.00 


\section{Plant Name/} Stream

** FERC Number 08772

GRANT LAKE

GRANT CR, TRAIL R

** Subtotal **

** FERC Number 08827

SHIP CREEK

CITY WATER \& WASTE WTR PIPELIN

** Subtotal **

** FERC Number 09996

REDISCOVERY LODGE

BELL CR

** Subtotal **

** FERC Number 10064

WHITTIER CREEK

WHITTIER CR, PASSAGE CANAI

** Subtotal **

** FERC Number 10524

POWER CREEK

POWER CR, EYAK LAKE

** Subtotal **

** FERC Number 10685A

CRATER LAKE

CRATER IAAKE, ORCA INLET

** Subtotal **

** FERC Number 10686

I.AKE REDFIELD

LAKE REDFIELD, YAKUTAT BAY

\section{Dam \\ ST Stat \\ Name Plate}

Rating (KW) PESF

PESF * KW

AK U

$830.00 \quad 0.25$

207.50

$$
830.00
$$

AK U

$510.00 \quad 0.90$

459.00

$$
510.00
$$

459.00

AK U

$$
115.00 \quad 0.25
$$

$$
115.00
$$

AK U

$200.00 \quad 0.25$

50.00

$$
200.00
$$

50.00

AK U

$6000.00 \quad 0.10$

600.00

6000.00

600.00

AK U

$600.00 \quad 0.25$

150.00

600.00

150.00

AK U

$2000.00 \quad 0.50$

1000.00 
HYDROPOWER RESOURCE ASSESSMENT BY FERC NUMBER

\section{Plant Name/ stream}

** Subtotal **

** FERC Number 11077 GOAT LAKE

SKAGWAY R(GOAT LAKE)

** Subtotal **

** FERC Number 11244

SIIVER ILAKE

DUCK R, GALENA BAY

** Subtotal **

** FERC Number 11316

TAZIMINA RIVER

TAZIMINA $R$

** Subtotal **

** FERC Number 11319

HAINES

UNN STREAM, CHILKOOT R

** Subtotal **

* * FERC Number 11393

MOHONEY LAKE

MAHONEY CR

** Subtotal **

** FERC Number 11497

LEANNE LAKE

LEANNE LAKE OUTLET

** Subtotal **

$\begin{array}{lll} & \text { Dam } & \text { Name Plate } \\ \text { ST Stat } & \text { Rating (KW) PESF } & \text { PESF * KW }\end{array}$

AK U

$6000.00 \quad 0.50$

3000.00

6000.00

3000.00

AK U

$15000.00 \quad 0.25$

3750.00

15000.00

3750.00

AK U

700.000 .10

70.00

700.00

70.00

AK U

$2500.00 \quad 0.25$

625.00

2500.00

625.00

AK U

$9000.00 \quad 0.25$

2250.00

9000.00

2250.00

AK U

$2800.00 \quad 0.90$

2520.00

2800.00

2520.00 
Plant Name/

stream

** FERC Number 11508

WOLF LAAKE

WOLF CR

** Subtotal **

* * FERC Number 11510

ALLISON

ALLISON CR

** Subtotal **

** FERC Number AKO01 BROWNE

NENANA R

** Subtotal **

** FERC Number AK005 GRANT I.AKE

WOOD $R$, KOKWOK $R$

** Subtotal **

** FERC Number AK010

TAZIMINA

TAZIMINA $R$

** Subtotal **

** FERC Number AK011

PORT LIONS

MENNONITE CR, SETTLERS COVE

** Subtotal **

** FERC Number AK012

CHAKACHAMNA

CHAKACHAMNA $\mathbf{R}$

$\begin{array}{lll} & \text { Dam } & \text { Name Plate } \\ \text { ST Stat } & \text { Rating (KW) PESF } & \text { PESF * KW }\end{array}$

AK U

$$
2500.00 \quad 0.25
$$

625.00

$$
2500.00
$$

625.00

AK U

$8000.00 \quad 0.25$

2000.00

$$
8000.00
$$

$$
2000.00
$$

AK U

$$
200000.00 \quad 0.10
$$

20000.00

200000.00

20000.00

AK U

$2700.00 \quad 0.25$

675.00

$$
2700.00
$$

675.00

AK U

$18000.00 \quad 0.10$

1800.00

$$
18000.00
$$

1800.00

$A K \quad U$

$$
200.00 \quad 0.25
$$

50.00

$$
200.00
$$

50.00

AK U

$366000.00 \quad 0.90$

329400.00 
6

HYDROPOWER RESOURCE ASSESSMENT BY FERC NUMBER

Plant Name/

Stream

** Subtotal **

** FERC Number AK018

TALKEETNA 2

TALKEETNA R

** Subtotal **

** FERC Number AK019

CHULITNA-HURRICANE

CHULITNA R

** Subtotal **

** FERC Number AK024

MAIN LAKE

MAIN BAY, PRINCE WILLIAM SOUND

** Subtotal **

** FERC Number AK026

KASNYKU LAKE

KASNYKU FALLS STR

** Subtotal **

** FERC Number AK027

CARBON LAKE

CARBON ILAKE

** Subtotal **

** FERC Number AKO28

MILK ILAKE

MILK CR

** Subtotal **

$\begin{array}{llll} & \text { Dam } & \text { Name Plate } & \\ \text { ST Stat } & \text { Rating (KW) PESF } & \text { PESF * KW }\end{array}$

$$
366000.00
$$

329400.00

AK U

$90000.00 \quad 0.25$

22500.00

90000.00

22500.00

AK U

$34000.00 \quad 0.25$

8500.00

34000.00

8500.00

$\mathrm{AK} U$

$125.00 \quad 0.25$

31.25

125.00

31.25

AK U

7000.00

0.50

3500.00

7000.00

3500.00

AK U

$10000.00 \quad 0.25$

2500.00

10000.00

2500.00

AK U

$7000.00 \quad 0.25$

1750.00

7000.00

1750.00 
HYDROPOWER RESOURCE ASSESSMENT BY FERC NUMBER

Plant Name/

stream

** FERC Number AK029

BORODINO LAKE

BIG PORT WALTER, CHATHAM STRAIT

** Subtotal **

** FERC Number AK030

BETTY LAKE

PORT ARMSTRONG, CHATHAM STRAIT

** Subtotal **

** FERC Number AK032

DIANA LAKE

UNN STR

** Subtotal **

** FERC Number AK034

PELICAN

PELICAN CR,LISIANSKI INLET

** Subtotal **

** FERC Number AK035

GOLD CREEK

GOLD CR

** Subtotal **

** FERC Number AK037

LAKE DOROTHY

DOROTHY CR

** Subtotal **

** FERC Number AK038

SPEEL DIVISION

SPEEL $R$

\section{Dam Name Plate \\ ST stat Rating (KW) PESF}

AK U

$5000.00 \quad 0.25$

$$
5000.00
$$

1250.00

AK U

$$
200.00
$$

0.25

50.00

$$
200.00
$$

50.00

AK U

$$
8000.00 \quad 0.25
$$

2000.00

$$
8000.00
$$

2000.00

AK U

1000.00

0.25

250.00

$$
1000.00
$$

250.00

AK U

2000.00

0.90

1800.00

$$
2000.00
$$

1800.00

AK U

$34000.00 \quad 0.50$

17000.00

34000.00

17000.00

AK U

$63000.00 \quad 0.25$

15750.00 
HYDROPOWER RESOURCE ASSESSMENT BY FERC NUMBER

\section{Plant Name/ \\ stream}

** Subtotal **

* * FERC Number AK039

CRATER LAKE

CRATER CR, SPEEL $R$

** Subtotal **

** FERC Number AK0 42

UPPER SWEETHEART

SWEETHEART FALLS CR

** Subtotal **

** FERC Number AK043

SCENERY

SCENERY CR

** Subtotal **

** FERC Number AK044

RUTH ILAKE

DELTA CR

** Subtotal **

** FERC Number AK049

HARDING

HARDING $\mathrm{R}$

** Subtotal **

** FERC Number AK055

LAKE CONNELL

WARD CR, WARD COVE

** Subtotal **

$\begin{array}{ll}\text { Dam } & \text { Name Plate } \\ \text { ST Stat Rating (KW) PESF } & \text { PESF * KW }\end{array}$

$$
63000.00
$$

15750.00

AK U

$27000.00 \quad 0.25$

6750.00

27000.00

6750.00

AK U

$7000.00 \quad 0.50$

3500.00

7000.00

3500.00

AK U

$15000.00 \quad 0.10$

1500.00

15000.00

1500.00

AK U

$13000.00 \quad 0.50$

6500.00

13000.00

6500.00

AK U

$18000.00 \quad 0.10$

1800.00

18000.00

1800.00

$\mathrm{AK} \mathrm{U}$

$2000.00 \quad 0.10$

200.00

2000.00

200.00 
9

HYDROPOWER RESOURCE ASSESSMENT BY FERC NUMBER

Plant Name/

stream $\begin{array}{ll}\text { Dam } & \text { Name Plate } \\ \text { ST } & \text { Stat } \\ \text { Rating (KW) PESF }\end{array}$

AK U

IAAKE MARY

OLD FRANKS CR

** Subtotal **

** FERC Number AK058

BLACK BEAR

BLACK BEAR CR

** Subtotal **

** FERC Number AK059

SCAMMON BAY

UNN STR, KUN R

** Subtotal **

** FERC Number AK062

PALMER

MCROBERTS CREEK

** Subtotal **

** FERC Number 10773

BURNETT R HATCHERY

BURNETT $R$

** Subtotal **

** FERC Number AK022

BRADLEY LAKE

BRADLEY CR

** Subtotal **

** FERC Number AK051

PURPLE IAKE

PURPLE ILAKE OUTLET
$A K \quad U$

$$
9600.00
$$

2400.00

5000.00

1250.00

AK U

$100.00 \quad 0.90$

90.00

$$
100.00
$$

90.00

AK U

$400.00 \quad 0.25$

100.00

400.00

100.00

AK W

$250.00 \quad 0.50$

125.00

250.00

125.00

AK W

$63900.00 \quad 0.90$

57510.00

63900.00

57510.00

AK W

$500.00 \quad 0.75$

375.00 
HYDROPOWER RESOURCE ASSESSMENT BY FERC NUMBER

\section{Plant Name/ \\ stream}

** Subtotal **

** FERC Number 01432

DRY SPRUCE

UNN TRIB, DRY SPRUCE BAY

** Subtotal **

** FERC Number 02251

SAN JUAN

SAN JUAN LAKE

** Subtotal **

** FERC Number 02267

PULP MILL

MEDVETCHA $R$

** Subtotal **

** FERC Number 02328

I.AKE WHITMAN

CASE CR

** Subtotal **

** FERC Number 03175

KISARAIIK

KISARALIK $R$

** Subtotal **

** FERC Number 04641

DANNER-FENSTER

UNN STR, GASTINEAU CHANNEL

** Subtotal **

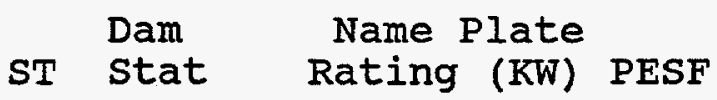

500.00

375.00

AK WO

$75.00 \quad 0.50$

37.50

75.00

37.50

AK WO

$100.00 \quad 0.75$

100.00

75.00

AK WO

$900.00 \quad 0.50$

450.00

900.00

450.00

AK WO

$2500.00 \quad 0.75$

1875.00

2500.00

1875.00

AK WO

$30000.00 \quad 0.50$

15000.00

30000.00

15000.00

AK WO

$12.00 \quad 0.50$

6.00

12.00

6.00 
HYDROPOWER RESOURCE ASSESSMENT BY FERC NUMBER

$$
\begin{gathered}
\text { Plant Name/ } \\
\text { stream }
\end{gathered}
$$

** FERC Number 06601

CALIFORNIA CREEK

CALIFORNIA CR, PLACER R

** Subtotal **

** FERC Number 07114A

DEVII CANYON

SUSITNA $R$

** Subtotal **

** FERC Number 07114B

WATANA

SUSITNA R

** Subtotal **

** FERC Number 07796 REGIONAL

MINOOK CR, YUKON R

** Subtotal **

** FERC Number 07799

TANANA

BEAR CR, YUKON R

** Subtotal **

** FERC Number 08756

JUNEAU CREEK

JUNEAU CR, KENAI R

** Subtotal **

** FERC Number 08765

BEAR CREEK

BEAR CR, TURNAGAIN ARM

$\begin{array}{lll} & \text { Dam } & \text { Name Plate } \\ \text { ST Stat } & \text { Rating (KW) PESF } & \text { PESF * KW }\end{array}$

AK Wo

$$
1500.00 \quad 0.75
$$

1125.00

$$
1500.00
$$

1125.00

AK Wo

$600000.00 \quad 0.50$

300000.00

$$
600000.00
$$

300000.00

AK Wo

1020000.00

0.50

510000.00

$$
1020000.00
$$

510000.00

AK WO

$2000.00 \quad 0.75$

1500.00

$$
2000.00
$$

1500.00

AK WO

$1000.00 \quad 0.75$

750.00

1000.00

750.00

AK Wo

$1800.00 \quad 0.50$

900.00

1800.00

900.00

AK wo

$250.00 \quad 0.50$

125.00 
HYDROPOWER RESOURCE ASSESSMENT BY FERC NUMBER

Plant Name/

Stream

\author{
Dam \\ ST stat
}

PESF * KW

** Subtotal **

250.00

125.00

** FERC Number 08766

STEEP CREEK

STEEP CR, EYAK R

AR wo

$870.00 \quad 0.75$

652.50

** Subtotal **

870.00

652.50

** FERC Number 08767

BEARTRAIL

BEARTRAIL CR, IBECK CR

AK Wo

$850.00 \quad 0.50$

425.00

** Subtotal **

850.00

425.00

** FERC Number 08768

LOST CREEK

LOST CR, SALMON CR, RESURRECTION

AK wo

$900.00 \quad 0.75$

675.00

** Subtotal **

900.00

675.00

** FERC Number 08769

KASIDAYA CREEK

KASIDAYA CREEK, TAIYA INLET

AK wo

$700.00 \quad 0.10$

70.00

** Subtotal **

$$
700.00
$$

70.00

** FERC Number 09760

TREADWELL DITCH

TREADWELL DH, GASTINEAU CHANNEL

AK wo

$2200.00 \quad 0.90$

1980.00

** Subtotal **

2200.00

1980.00

** FERC Number 09763

NUGGET CREEK

NUGGET CR, MENDENHAL LK

AR wo

$2200.00 \quad 0.50$

1100.00

** Subtotal ** 


\section{Plant Name/}

Stream

\author{
Dam \\ ST \\ Name Plate \\ Rating (KW) PESF
}

SHEEP CREEK

SHEEP CR, TAKU INLET

** Subtotal **

** FERC Number 10685B

CRATER LAKE

CRATER IAAKE, ORCA INLET

** Subtotal **

** FERC Number 11139

TERROR IAAKE

TERROR R

** Subtotal **

** FERC Number 11243

POWER CREEK

POWER CR, EYAK LAKE

** Subtotal **

** FERC Number 11287

LACE RIVER

UNN STR, EVELYN LAKE

** Subtotal **

** FERC Number 11290

TAIYA INLET

DAYEBAS CR, KATZEHIN $R$
150.00

AK WO

AK Wo

$$
150.00 \quad 0.75
$$

112.50

1550.00

$$
3100.00
$$

1550.00

AK wo

$1000.00 \quad 0.75$

750.00

$$
1000.00
$$

750.00

AK wo

$3000.00 \quad 0.75$

2250.00

3000.00

2250.00

AK Wo

$5000.00 \quad 0.50$

2500.00

5000.00

2500.00

AK WO

$4900.00 \quad 0.75$

3675.00

4900.00

3675.00

AK Wo

$2000.00 \quad 0.75$

1500.00 
HYDROPOWER RESOURCE ASSESSMENT BY FERC NUMBER

Plant Name/

Stream $\begin{array}{ll}\text { Dam } & \text { Name Plate } \\ \text { ST Stat } & \text { Rating (KW) PESF }\end{array}$

SNYDER FALLS CREEK

SNYDER FALLS CR, NELSON BAY

** Subtotal **

** FERC Number 11480

UPPER REYNOLDS CREEK

REYNOLDS CR, COPPER HARBOR

** Subtotal **

** FERC Number AKOO2

SCAMMON

UNN STR, SCAMMON BAY

** Subtotal **

** FERC Number AKOO3

SCAMMON (PHASE 2)

UNN STR, SCAMMON BAY

** Subtotal **

** FERC Number AKOO4

ILAKE ELVA

ELVA CR

** Subtotal **

** FERC Number AK006

NUYAKUK (TIKCHIK)

NUYAKUK $R$

** Subtotal **
2000.00

AK WO

AK wo

PESF * $\mathrm{KW}$

1500.00

$1450.00 \quad 0.75$

1087.50

1450.00

1087.50

AK Wo

$2000.00 \quad 0.50$

1000.00

2000.00

1000.00

AK WO

$175.00 \quad 0.90$

157.50

175.00

157.50

AK wo

$125.00 \quad 0.90$

112.50

125.00

112.50

AK wo

$1500.00 \quad 0.75$

1125.00

1500.00

1125.00

$127000.00 \quad 0.75$

95250.00

127000.00

95250.00 


\section{Plant Name/ Stream}

** FERC Number AK007 AKUTAN UNN STR

** Subtotal **

** FERC Number AK008 INDIAN CREEK

INDIAN CR, CHIGNIK BAY

** Subtotal **

** FERC Number AKO09

ILIAMNA

KVICHAK R

** Subtotal **

** FERC Number AK013

COFFEE

BELUGA $\mathbf{R}$

** Subtotal **

** FERC Number AK014

UPPER BELUGA

BELUGA $R$

** Subtotal **

** FERC Number AK015

YENTNA

YENTNA $R$

** Subtotal **

** FERC Number AK016

SKWENTNA (HAYES)

SKWENTNA $R$

$\begin{array}{lll}\text { Dam } & \text { Name Plate } & \\ \text { STat } & \text { Rating (KW) PESF } & \text { PESF * KW }\end{array}$

AK Wo

$$
170.00 \quad 0.90
$$

153.00

170.00

153.00

AK Wo

$1100.00 \quad 0.10$

110.00

1100.00

110.00

AK Wo

$313000.00 \quad 0.50$

156500.00

313000.00

156500.00

AK wo

37000.00

0.75

27750.00

37000.00

27750.00

AK WO

$48000.00 \quad 0.75$

36000.00

48000.00

36000.00

AK WO

$219000.00 \quad 0.75$

164250.00

219000.00

164250.00

AR wo

$98000.00 \quad 0.75$

73500.00 
Plant Name/ Stream

** Subtotal **

** FERC Number AK017

KEETNA (TALKEETNA)

TALKEETNA $\mathrm{R}$

** Subtotal **

** FERC Number AK020

SNOW

SNOW $\mathbf{R}$

** Subtotal **

** FERC Number AKO2I SELDOVIA

FISH CR

** Subtotal **

** FERC Number AK023 LOWELL

LOWELL CR, DAY HARBOR

** Subtotal **

** FERC Number AK025 TAKATZ

TAKATZ CR, TAKATZ BAY

** Subtotal **

** FERC Number AK031 MAKSOUTOF

MAKSOUTOF $R$

** Subtotal **

$\begin{array}{ll}\text { Dam } & \text { Name Plate } \\ \text { ST Stat Rating (KW) PESF } & \text { PESF * KW }\end{array}$

AK WO

98000.00

73500.00

$74000.00 \quad 0.75$

55500.00

74000.00

55500.00

AK WO

$63000.00 \quad 0.50$

31500.00

63000.00

31500.00

AK Wo

$460.00 \quad 0.50$

230.00

460.00

230.00

AK Wo

$650.00 \quad 0.75$

487.50

650.00

487.50

AK Wo

$20000.00 \quad 0.75$

15000.00

20000.00

15000.00

AK WO

$24000.00 \quad 0.50$

12000.00

24000.00

12000.00 
HYDROPOWER RESOURCE ASSESSMENT BY FERC NUMBER

$$
\begin{aligned}
& \text { Plant Name/ } \\
& \text { Stream }
\end{aligned}
$$

** FERC Number AK033

SUPERIOR

ELFIN COVE (WACHUSETT COVE CR)

** Subtotal **

** FERC Number AK036

ANNEX CREEK

ANNEX CR, TAKU INLET

** Subtotal **

** FERC Number AK040

TEASE

LONG $\mathrm{R}$

** Subtotal **

** FERC Number AK041 SWEETHEART FALLS

SWEETHEART FALLS CR

** Subtotal **

** FERC Number AK045

GOAT

GOAT CR

** Subtotal **

** FERC Number AK046

BLIND SLOUGH

CRYSTAL CR,BLIND R

** Subtotal **

** FERC Number AK047

ANITA LAKE

ANITA CR, KUNK CR $\begin{array}{lll}\text { Dam } & \text { Name Plate } & \\ \text { ST Stat } & \text { Rating (KW) PESF } & \text { PESF * KW }\end{array}$

AK Wo

$750.00 \quad 0.75$

562.50

750.00

562.50

AK WO

$1750.00 \quad 0.50$

875.00

1750.00

875.00

AK WO

$16000.00 \quad 0.75$

12000.00

16000.00

12000.00

AK Wo

$29000.00 \quad 0.50$

14500.00

29000.00

14500.00

AK Wo

$20000.00 \quad 0.75$

15000.00

20000.00

15000.00

AK Wo

$1400.00 \quad 0.50$

700.00

1400.00

700.00

AK WO

$3230.00 \quad 0.50$

1615.00 
HYDROPOWER RESOURCE ASSESSMENT BY FERC NUMBER

\section{Plant Name/} stream

** Subtotal **

** FERC Number AK048

VIRGINIA LAKE

MILL CR

** Subtotal **

** FERC Number AK050

IAKE GRACE

GRACE CR, BEHM CANAL

** Subtotal **

** FERC Number AK052

MAHONEY LAKE

MAHONEY CR

** Subtotal **

** FERC Number AK053

UPPER SILVIS

BEAVER FALIS CR, GEORGE INLET

** Subtotal **

** FERC Number AK054

KETCHIKAN LAKES

KETCHIKAN CR

** Subtotal **

** FERC Number AK056

IINKUM

LINKUM CR, KASAAN BAY

** Subtotal **

$\begin{array}{lll}\text { Dam } & \text { Name Plate } & \\ \text { ST Stat } & \text { Rating (KW) PESF } & \text { PESF * KW }\end{array}$

$$
3230.00
$$

1615.00

AK WO

$12000.00 \quad 0.50$

6000.00

12000.00

6000.00

AK Wo

$21000.00 \quad 0.50$

10500.00

21000.00

10500.00

AK WO

$14400.00 \quad 0.75$

10800.00

14400.00

10800.00

AK Wo

$20000.00 \quad 0.50$

10000.00

20000.00

10000.00

AK Wo

$2000.00 \quad 0.50$

1000.00

2000.00

1000.00

AK WO

$4560.00 \quad 0.75$

3420.00

4560.00

3420.00 
Plant Name/ stream

** FERC Number AK060

PYRAMID CREEK

PYRAMID CR, PACIFIC OCEAN

** Subtotal **

** FERC Number AK061

SOUTH FORK EAGLE RIVER

$S$ FK EAGLE R

** Subtotal **

*** Total ***

$\begin{array}{lll}\text { Dam } & \text { Name Plate } & \\ \text { ST Stat } & \text { Rating (KW) PESF } & \text { PESF * KW }\end{array}$

AR wo

$1400.00 \quad 0.90$

1260.00

1400.00

1260.00

AK Wo

$1100.00 \quad 0.75$

825.00

1100.00

825.00

4042257.00 
Appendix D

Individual Resource Database List 
DATE： $10 / 31 / 97$

FERC

Number

Plant Name

01432

DRY SPRUCE

County Name

KODIAK DIVISION

Class

Owner Name

I

WARDS COVE PACKING CO

Name Plate

Rating ( $\mathrm{KW}$ )

PESF

Annual Energy

PESF*KW

Rating (MWh)

75.00

0.50

37.50

300.00

PAGE NO: 1

UNN TRIB, DRY SPRUCE BAY

River Basin

KODIAK ISLAND RIVER BASINS state

Name

AK

$\begin{array}{ccccc}\text { Unit } & \text { Plant } & \text { Project } & \text { Dam } & \text { Latitude } \\ \text { Type } & \text { Type } & \text { Status } & \text { Status } & \text { Longitude } 15303.00 \\ \text { C } & \text { DIV } & \text { No } & \text { WO } & \end{array}$

Factor

Wild/Scenic Protection

Wild/Scenic Tributary or

Upstream/Downstream

wild/Scenic Location

Cultural value

Fish Presence Value

Geologic Value

Historic Value

other Value

Recreation Value

Scenic value
Exist Prob

0.90

wildlife Value Threatened/Endangered Fish Threatened/Endangered Wildlife

0.90 Federal Land Code 103

0.90 Federal Land Code 104

Y $\quad 0.75$ Federal Land Code 105

0.90 Federal Land Code 106

0.90 Federal Land Code 107

0.90 Federal Land Code 108

0.90 Federal Land Code 198
PESF Annual Energy Rating (MWh)

150

0.90

Exist Prob

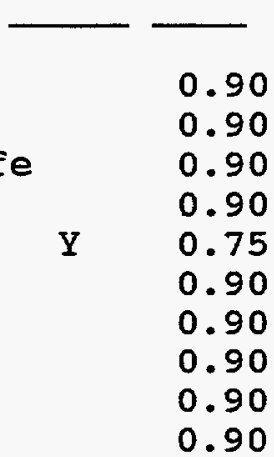


R E S O U R C E D A T A B A S E I I S T I N G

DATE: $10 / 31 / 97$

PAGE NO: 2

FERC

Number

Plant Name

stream

State

Name

02251 SAN JUAN

SAN JUAN LARE

AK

County Name

VALDEZ-CHITINA-WHITT DV
River Basin

KENAI PENINSULA RIVER BAS

class Owner Name

I NEW ENGLAND FISH CO

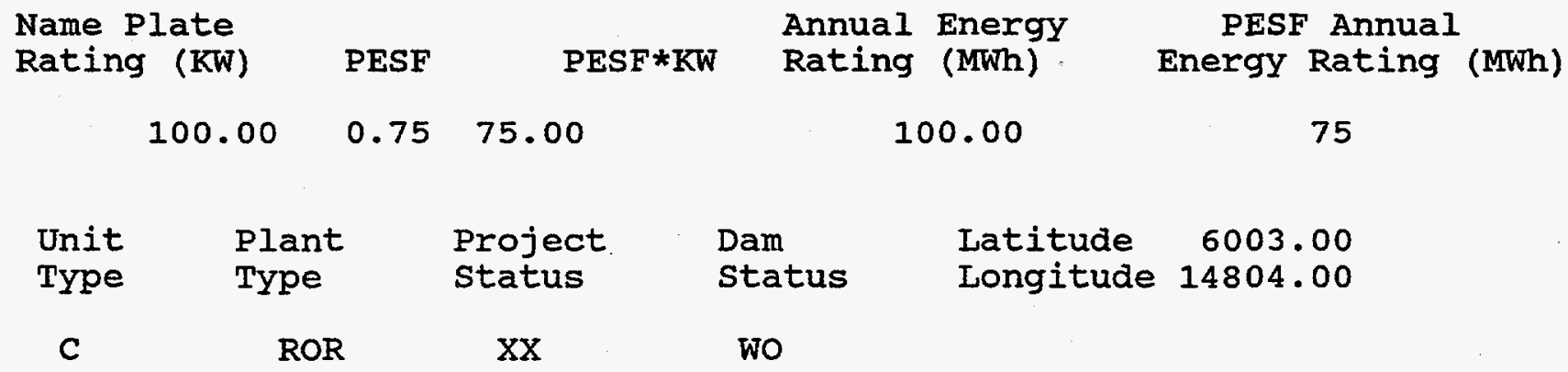

Factor

Exist Prob

0.90 Wildife value Threatened/Endangered Fish Threatened/Endangered Wildlife

0.90 Federal Land Code 103

0.90 Federal Land Code 104

Y $\quad 0.75$ Federal Land Code 105

0.90 Federal Land Code 106

0.90 Federal Land Code 107

0.90 Federal Land Code 108

0.90 Federal Land Code 198

0.90

Historic Value

other Value

Recreation Value

Scenic Value
Factor

Exist Prob

0.90

0.90

0.90

0.90

0.90

0.90

0.90

0.90

0.90

0.90 
DATE: $10 / 31 / 97$

FERC

Number

Plant Name

02267 PULP MILL

County Name

SITKA DIVISION
PAGE NO: 3

State

Name

AK
MEDVETCHA R

River Basin

JUNEAU GROUP

\section{Class Owner Name \\ I ALASKA PULP CORP}

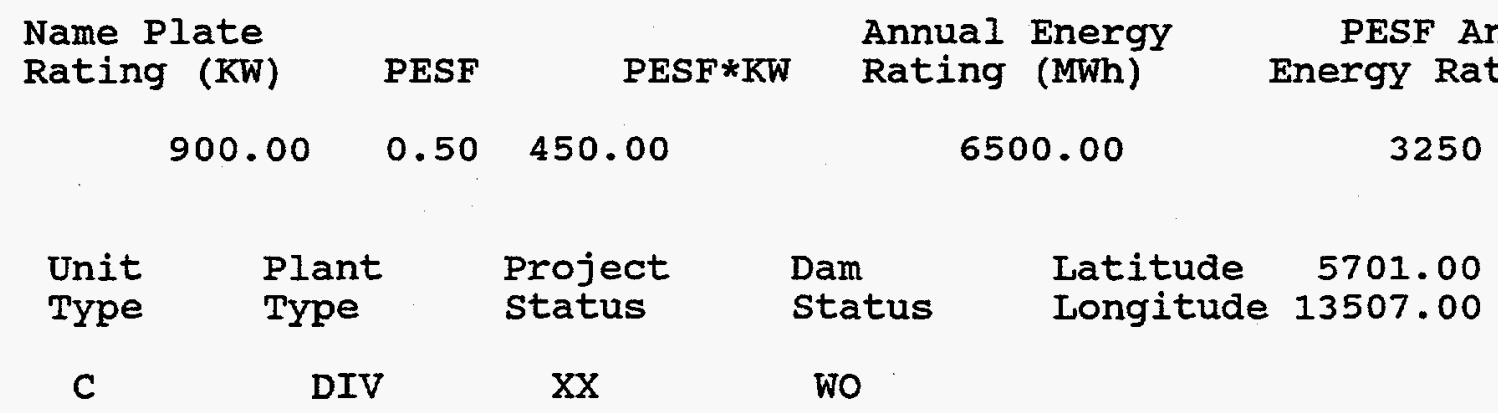

Factor

Wild/Scenic Protection

wild/Scenic Tributary or Upstream/ Downstream wild/Scenic Location Cultural Value Fish Presence Value Geologic Value Historic Value other Value Recreation Value Scenic Value
Exist Prob

(2)

Exist Prob

Factor

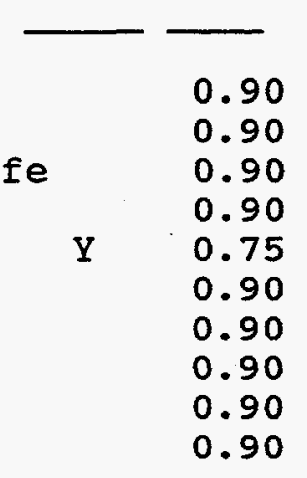



R E S O U R C E
D A T A B A S E
L I S T I N G

DATE: $10 / 31 / 97$

PAGE NO: 4

FERC

Number

Plant Name

stream

State

Name

02328

I.AKE WHITMAN

CASE CR

AK

county Name

River Basin

KETCHIKAN DIVISION

KETCHIKAN GROUP

Class Owner Name

M KETCHIKAN, CITY OF

Name Plate

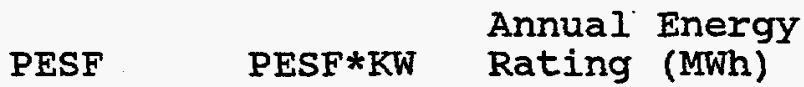

PESF Annual

Rating (KW)

$0.75 \quad 1875.00$

11600.00

Energy Rating (MWh)

2500.00

8700

Unit Plant

Type Type

C

DIV

Factor

Wild/Scenic Protection

Wild/Scenic Tributary or Upstream/Downstream wild/Scenic Location Cultural Value Fish Presence Value

Geologic Value

Historic Value other value

Recreation Value

Scenic Value
Project status

$\mathrm{XX}$

Exist Prob

0.90

Wildlife Value

Threatened/Endangered Fish Threatened/Endangered Wildife

0.90 Federal Land Code 103

0.90 Federal Land Code 104

0.90 Federal Land Code 105

0.90 Federal Land Code 106

0.90 Federal Land Code 107

0.90 Federal Land Code 108

0.90 Federal Land Code 198

0.90
Exist Prob

0.90

0.90

0.90

0.90

0.75

0.90

0.90

0.90

0.90

0.90 
DATE: $10 / 31 / 97$

FERC

Number

Plant Name

02755A THOMAS BAY

County Name

WRANGELL-PETERSBURG DIV
PAGE NO: 5
State

Name

AK

THOMAS BAY

stream

River Basin

JUNEAU GROUP

$\begin{array}{cc}\text { Class } & \text { Owner Name } \\ \text { M } & \text { THOMAS BAY POWER COMM }\end{array}$

Name Plate PESF

Annual Energy Rating ( $\mathrm{KW})$ PESF*KW Rating (MWh)

PESF Annual 30000.00 0.257500 .00 130000.00 Energy Rating (MWh) 32500

\section{Unit} Type

$$
\text { Plant }
$$
Type

c DIV

\section{Project status}

$\mathrm{Xx}$

Exist Prob

Factor

Wild/Scenic Protection Wild/Scenic Tributary or Upstream/Downstream wild/Scenic Location Cultural value Fish Presence Value Geologic Value Historic Value other value Recreation value Scenic Value

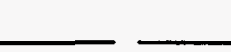

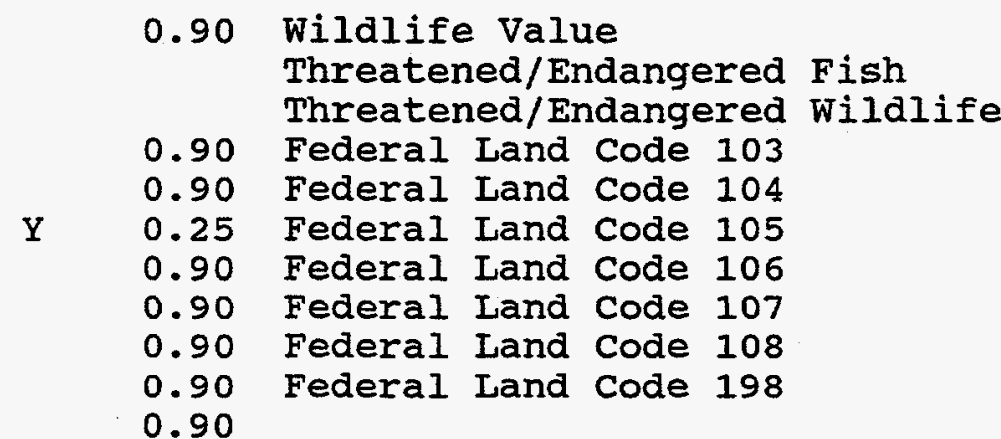
Latitude $\quad 5707.00$ Longitude 13242.00
Dam status

U
Factor

Exist Prob

0.90
0.90
0.90
0.90
0.50
0.90
0.90
0.90
0.90
0.90


R E S O U R C E

DATE: $10 / 31 / 97$
D A T A B A S E

FERC

Number

$02755 B$

FALLS IAAKE

County Name

WRANGELL-PETERSBURG DIV
I I S T I N G

PAGE NO: 6
State

Name

CASCADE CR, THOMAS BAY

River Basin

JUNEAU GROUP
AK

\section{Class Owner Name \\ M THOMAS BAY POWER COMM}

\begin{tabular}{|c|c|c|c|c|c|c|}
\hline $\begin{array}{l}\text { Name } F \\
\text { Rating }\end{array}$ & & PESF & $P E S F * K W$ & $\begin{array}{l}\text { Ann } \\
\text { Rat }\end{array}$ & $\begin{array}{l}\text { Energy } \\
\text { (MWh) }\end{array}$ & $\begin{array}{l}\text { PESF Annual } \\
\text { EnergY Rating (MWh) }\end{array}$ \\
\hline & .00 & 0.10 & 800.00 & & 00.00 & 3500 \\
\hline $\begin{array}{l}\text { Unit } \\
\text { Type }\end{array}$ & $\begin{array}{l}\text { P1 } \\
\text { Ty }\end{array}$ & & $\begin{array}{l}\text { Project } \\
\text { status }\end{array}$ & $\begin{array}{l}\text { Dam } \\
\text { status }\end{array}$ & $\begin{array}{l}\text { Latitude } \\
\text { Longitude }\end{array}$ & $\begin{array}{r}5700.00 \\
-13245.00\end{array}$ \\
\hline C & & & $\mathrm{xX}$ & $\mathrm{U}$ & & \\
\hline
\end{tabular}

Factor

wild/Scenic Protection

Wild/Scenic Tributary or Upstream/Downstream wild/Scenic Location Cultural value

Fish Presence Value Geologic Value Historic Value other Value Recreation Value Scenic Value
Exist Prob

(1)

\section{Factor}

Exist Prob

0.90 Wildlife Value Threatened/Endangered Fish Threatened/Endangered Wildlife

0.90

$\begin{array}{ll}0.90 & \text { Federal Land Code } 103 \\ 0.90 & \text { Federal Land Code } 104\end{array}$

Y $\quad 0.25$ Federal Land Code 105

0.90 Federal Land Code 106

0.90 Federal Land Code 107

0.90 Federal Land Code 108

$\mathrm{Y} \quad 0.25$ Federal Land Code 198

$\begin{array}{ll}\mathrm{Y} & 0.25 \\ \mathrm{Y} & 0.50\end{array}$ 
DATE: $10 / 31 / 97$

FERC

Number

Plant Name

02760

PAGE NO: 7

state

Name

POWER CR, EYAK LAKE

AK

county Name

CORDOVA-MCCARTHY DIV
River Basin

KENAI PENINSULA RIVER BAS

\section{Class Owner Name}

M CORDOVA PUBLIC UTILITIES

$\begin{array}{llcccc}\begin{array}{l}\text { Name Plate } \\ \text { Rating (KW) }\end{array} & \text { PESF } & \text { PESF*KW } & \begin{array}{c}\text { Annual Energy } \\ \text { Rating (MWh) }\end{array} & \begin{array}{c}\text { PESF Annual } \\ \text { Energy Rating (MWh) }\end{array} \\ 5000.00 & 0.10 & 500.00 & 26000.00 & 2600\end{array}$

$\begin{array}{llllr}\text { Unit } & \text { Plant } & \text { Project } & \text { Dam } & \text { Latitude } \\ \text { Type } & \text { Type } & \text { Status } & \text { Status } & \text { Longitude } 14532.00\end{array}$

C

DIV

$\mathrm{XX}$

$\mathrm{U}$

Factor

wild/Scenic Protection

wild/Scenic Tributary or

Upstream/Downstream

wild/Scenic Location

Cultural value

Fish Presence Value

Geologic Value

Historic Value

other value

Recreation Value

Scenic Value
Exist Prob

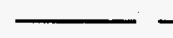

$\begin{array}{cll} & 0.90 & \begin{array}{l}\text { Wildlife Value } \\ \text { Threatened/Endangered }\end{array} \\ & & \text { Threatened/Endangered } \\ & 0.90 & \text { Federal Land Code } 103 \\ & 0.90 & \text { Federal Land Code } 104 \\ \text { Y } & 0.25 & \text { Federal Land Code } 105 \\ & 0.90 & \text { Federal Land Code } 106 \\ & 0.90 & \text { Federal Land Code } 107 \\ & 0.90 & \text { Federal Land Code } 108 \\ & 0.25 & \text { Federal Land Code } 198 \\ & & \end{array}$

Exist Prob

Factor

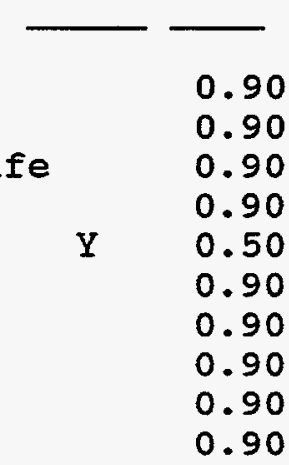


R E S O U R C E D A T A B A S E I I S T I N G

DATE: $10 / 31 / 97$

PAGE NO: 8

FERC

Number

Plant Name

Stream

State

Name

03175

KISARALIK

KISARALIK R

AK

County Name

BETHEL DIVISION

Class

M ATIASKA ENERGY AUTH

Name Plate

Rating (KW)

30000.00

0.50

15000.00

\section{Plant}

Type

\section{Project} status

C

ROR

XX

Exist Prob

Factor

Wild/Scenic Protection

wild/Scenic Tributary or

Upstream/Downstream

wild/Scenic Location

Cultural value

Fish Presence Value

Geologic Value

Historic Value

other Value

Recreation value

Scenic Value

WO
River Basin

KOTZEBUE-NUSHAGAK RIVER B
PESF Annual

Annual Energy Rating (MWh)

131400.00

65700
Energy Rating (MWh)

Dam

Status

Latitude 6026.00

Longitude 16006.00

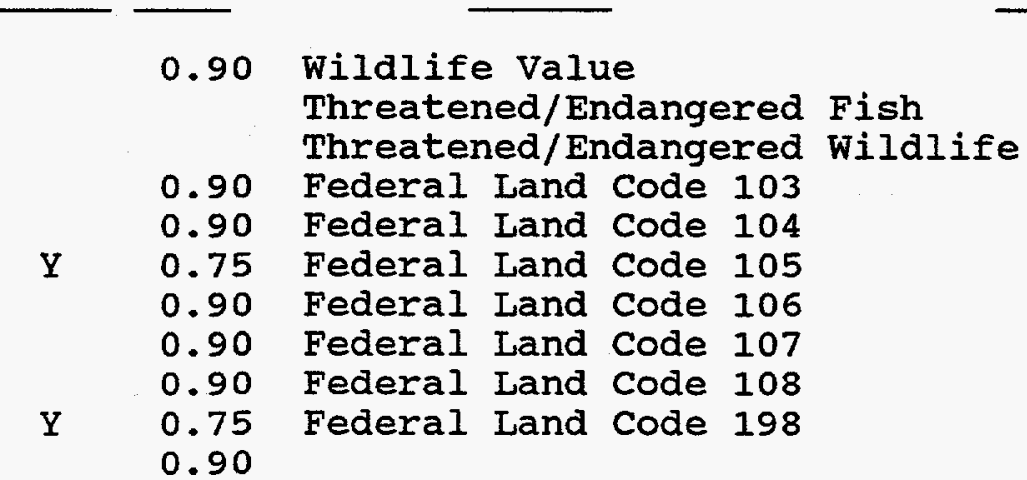

Exist Prob

Factor

Exist Prob

0.90

0.90

0.90

0.90

0.90

0.90

0.90

0.90

0.90

0.90 
R E S O U R C E

DATE: $10 / 31 / 97$
D A T A B A S E

D A A B A S

FERC

Number

Plant Name

04641

DANNER-FENSTER

County Name

JUNEAU DIVISION

class

Owner Name

$\mathbf{R}$

DANNER, GEORGE

Name Plate

Rating (KW)

PESF
PESF*KW Annual Energy
Rating (MWh)

6.00

Project

Unit Plant

Type Type

C

ROR

Factor

Exist Prob

Factor
12.00

Latitude

Longitude 13424.00
Dam status

WO
6

$\mathrm{xx}$ wo

PAGE NO: 9

stream

\author{
AK \\ UNN STR, GASTINEAU CHANNEL \\ . \\ River Basin \\ JUNEAU GROUP
}

State

Name
PESF Annual Energy Rating (MWh) wild/scenic Protection

wild/Scenic Tributary or

Upstream/Downstream

wild/Scenic Location

Cultural value

Fish Presence Value

Geologic Value

Historic Value

other Value

Recreation Value

Scenic Value 
FERC

Number

Plant Name

05212 THUMB CREEK

County Name

KETCHIKAN DIVISION
State

Name

THUMB CR, SALMON $R$

AK

\section{class Owner Name \\ R HYDER HYDRO CO}

Name Plate

Rating (KW)

10000.00

PESF

0.252500 .00

Unit

Type

$$
\begin{gathered}
\text { Plant } \\
\text { Type } \\
\text { ROR }
\end{gathered}
$$

C

$$
\begin{aligned}
& \text { Project } \\
& \text { status }
\end{aligned}
$$

$\mathrm{XX}$
Annual Energy Rating (MWh)

$$
57000.00
$$

PESF Annual Energy Rating (MWh)

14250
Factor

Wild/Scenic Protection

Wild/Scenic Tributary or Upstream/Downstream wild/Scenic Location Cultural Value Fish Presence Value Geologic Value Historic Value other value Recreation value Scenic Value
Exist Prob

Factor

Latitude $\quad 5555.00$

status Longitude 13002.00

U

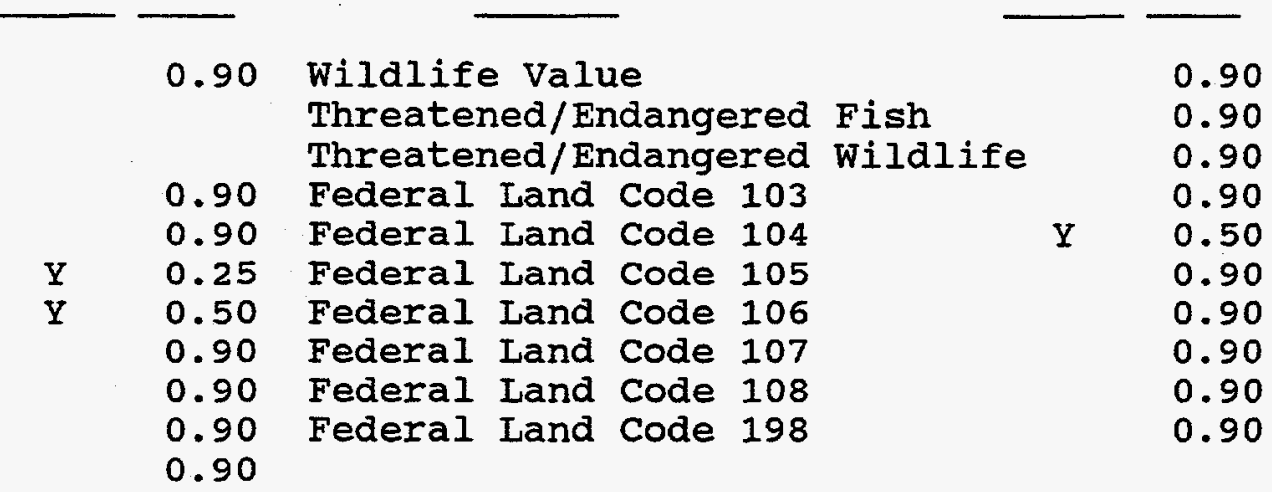


DATE: $10 / 31 / 97$

FERC

Number

05214
Plant Name

WEST MT HINCHLEY

county Name

KETCHIKAN DIVISION
PAGE NO: 11

State

Name

W HINCKLEY RUN, SALMON R

AK $\begin{array}{cr}\text { Class } & \text { Owner Name } \\ \text { R } & \text { HYDER HYDRO CO }\end{array}$

Name Plate

Rating ( $\mathrm{KW})$

2600.00

PESF

Annual Energy

PESF*KW Rating (MWh)

13000.00

$0.25 \quad 650.00$

KETCHIKAN GROUP

\begin{tabular}{|c|c|c|c|c|}
\hline $\begin{array}{l}\text { Unit } \\
\text { Type }\end{array}$ & $\begin{array}{l}\text { Plant } \\
\text { Type }\end{array}$ & $\begin{array}{l}\text { Project } \\
\text { Status }\end{array}$ & $\begin{array}{l}\text { Dam } \\
\text { status }\end{array}$ & $\begin{array}{lr}\text { Latitude } & 5553.00 \\
\text { Longitude } & 13002.00\end{array}$ \\
\hline C & ROR & $x x$ & U & \\
\hline
\end{tabular}

Factor

Wild/Scenic Protection

Wild/Scenic Tributary or

Upstream/Downstream

wild/Scenic Location

Cultural value

Fish Presence Value

Geologic Value

Historic Value

other Value

Recreation value

Scenic Value
PESF Annual Energy Rating (MWh) 3250
Exist Prob

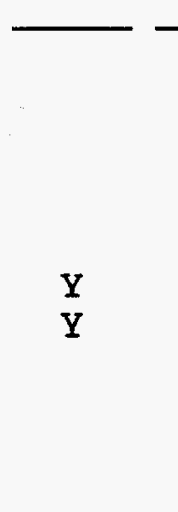

\section{Factor}

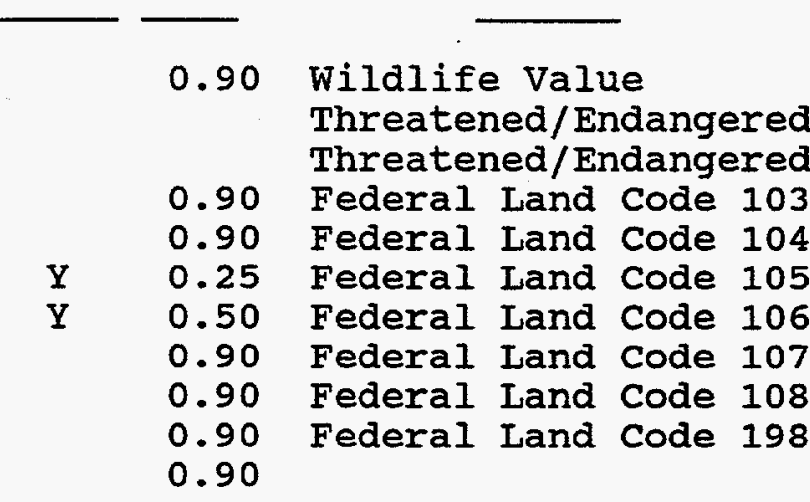

Exist Prob 
DATE: $10 / 31 / 97$

PAGE NO: 12

FERC

Number

Plant Name

stream

State

05215 FISH CREEK

FISH CR, SALMON R

Name

County Name

River Basin

KETCHIKAN DIVISION

KETCHIKAN GROUP

Class Owner Name

R HYDER HYDRO CO

Name Plate

Rating ( $\mathrm{KW}$ )

PESF

Annual Energy

PESF Annual

1300.00

0.25325 .00

8000.00

Energy Rating (MWh)

$\begin{array}{ll}\text { Unit } & \text { Plant } \\ \text { Type } & \text { Type }\end{array}$

c

ROR
Project status

$\mathrm{XX}$

Exist Prob

Factor

wild/Scenic Protection

wild/Scenic Tributary or Upstream/Downstream wild/Scenic Location Cultural value Fish Presence Value Geologic Value Historic Value other value Recreation value Scenic value
0.90

Wildlife value Threatened/Endangered Fish Threatened/Endangered Wildlife

0.90 Federal Land code 103

0.90 Federal Land Code 104

Y $\quad 0.25$ Federal Land Code 105

$\mathrm{Y} \quad 0.50$

0.90

0.90

0.90

0.90
Federal Land Code 106

Federal Land code 107

Federal Land Code 108

Federal Land Code 198

AK

\section{0}

Dam Latitude 5551.00

$\mathrm{U}$

Exist Prob

0.90

0.90

0.90

0.90

0.50

0.90

0.90

0.90

0.90

0.90 
DATE: $10 / 31 / 97$

PAGE NO: 13

FERC

Number

05216
Plant Name

ALYESKA CREEK

county Name

ANCHORAGE DIVISION stream

ALYESKA CR, GLACIER CR

River Basin

KENAI PENINSULA RIVER BAS
State

Name

AK

\section{Class Owner Name}

R ALYESKA HYDRO CO

Name Plate

Rating ( $\mathrm{KW})$

PESF

Annual Energy

1500.00

$0.25 \quad 375.00$

7800.00

PESF Annual

PESF*KW

Rating (MWh)

Energy Rating (MWh)

\subsection{0}

$\begin{array}{cccllr}\text { Unit } & \text { Plant } & \text { Project } & \text { Dam } & \text { Latitude } & 6122.00 \\ \text { Type } & \text { Type } & \text { Status } & \text { Status } & \text { Longitude } 14942.00 \\ \text { C } & \text { ROR } & \text { XX } & U & \end{array}$

Factor

Exist Prob

Factor

Exist Prob

Wild/Scenic Protection

wild/Scenic Tributary or

Upstream/Downstream

.

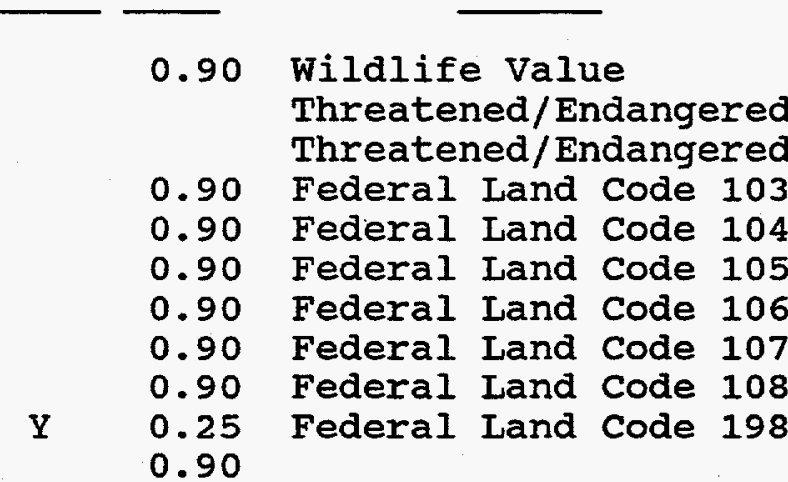

0.90

0.90

0.90

wild/Scenic Location

Cultural value

Fish Presence Value

0.90 Federal Land Code 104

0.90

0.90

Geologic Value

Historic Value

other Value

Recreation value

Scenic Value

0.90 Federal Land Code 106

0.90

0.90

0.90

0.90

0.90 
DATE: $10 / 31 / 97$

PAGE NO: 14

FERC

Number

06211 THOMAS BAY

County Name

WRANGELL-PETERSBURG DIV

Plant Name

\section{Class}

I

ALASKA INDUSTRIAL POWER CORP

Name Plate

Rating ( $\mathrm{KW}$ )

PESF

$0.10 \quad 3400.00$

$0.10 \quad 3400.00$

34000.00

CASCADE CR

stream

River Basin

JUNEAU GROUP
State

Name

AK

\begin{tabular}{|c|c|c|c|c|}
\hline $\begin{array}{l}\text { Unit } \\
\text { Type }\end{array}$ & $\begin{array}{l}\text { Plant } \\
\text { Type }\end{array}$ & $\begin{array}{l}\text { Project } \\
\text { Status }\end{array}$ & $\begin{array}{l}\text { Dam } \\
\text { status }\end{array}$ & $\begin{array}{lr}\text { Latitude } 5704.00 \\
\text { Longitude } 13246.00\end{array}$ \\
\hline C & ROR & $x X$ & $\mathrm{U}$ & \\
\hline
\end{tabular}

Factor

wild/Scenic Protection

wild/Scenic Tributary or Upstream/Downstream wild/Scenic Location cultural Value Fish Presence Value Geologic Value Historic Value other Value Recreation value Scenic Value
Exist Prob

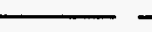

$\begin{array}{cc} & 0.90 \\ & \\ & \\ & 0.90 \\ & 0.90 \\ \mathrm{Y} & 0.25 \\ & 0.90 \\ & 0.90 \\ & 0.90 \\ \mathrm{Y} & 0.25 \\ \mathrm{Y} & 0.50\end{array}$

Factor

Exist Prob

Annual Energy

170000.00
17000

PESF Annual Energy Rating (MWh) 
DATE: $10 / 31 / 97$

PAGE NO: 15

FERC

Number Plant Name

stream

state

Number

CALIFORNIA CREEK

CALIFORNIA CR, PLACER R

Name

06601

County Name

River Basin

ANCHORAGE DIVISION

KENAI PENINSULA RIVER BAS

Class Owner Name

R TURNAGAIN HYDRO CO

Name Plate

Rating (KW)

PESF

Annual Energy

1500.00

$0.75 \quad 1125.00$

Rating (MWh)

PESF Annual

AK

Energy Rating (MWh)

6800.00

5100

\begin{tabular}{|c|c|c|c|c|}
\hline $\begin{array}{l}\text { Unit } \\
\text { Type }\end{array}$ & $\begin{array}{l}\text { Plant } \\
\text { Type }\end{array}$ & $\begin{array}{l}\text { Project } \\
\text { status }\end{array}$ & $\begin{array}{l}\text { Dam } \\
\text { Status }\end{array}$ & $\begin{array}{lr}\text { Latitude } & 6035.00 \\
\text { Longitude } 14853.00\end{array}$ \\
\hline C & ROR & $\mathrm{xx}$ & Wo & \\
\hline
\end{tabular}

Factor

Wild/Scenic Protection

Wild/Scenic Tributary or

Upstream/Downstream

wild/Scenic Location

Cultural Value

Fish Presence Value

Geologic Value

Historic Value

other Value

Recreation value

Scenic Value
Exist Prob

0.90

Wildlife Value

Threatened/Endangered Fish

Threatened/Endangered Wildlife

0.90 Federal Land Code 103

0.90 Federal Land Code 104

0.90 Federal Land Code 105

0.90 Federal Land Code 106

0.90 Federal Land Code 107

0.90 Federal Iand Code 108

0.90 Federal Land Code 198
Exist Prob

$\begin{array}{cc} & \\ & \\ & 0.90 \\ & 0.90 \\ & 0.90 \\ \mathrm{Y} & 0.90 \\ & 0.75 \\ & 0.90 \\ & 0.90 \\ & 0.90 \\ & 0.90 \\ & 0.90\end{array}$




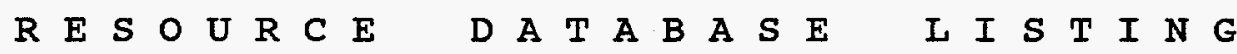

DATE : $10 / 31 / 97$

PAGE NO: 16

FERC

Number

Plant Name

stream

state

06856 THOMAS BAY

CASCADE CR

Name

06856

County Name

River Basin

WRANGELL-PETERSBURG DIV JUNEAU GROUP

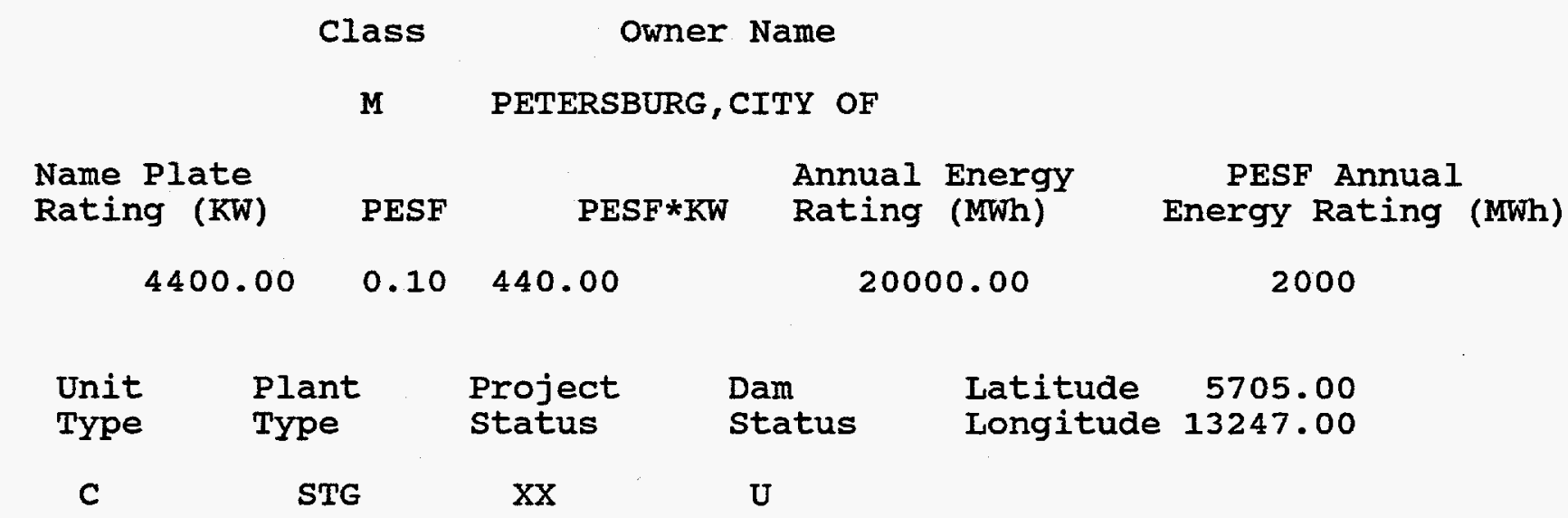

Factor

Exist Prob

Factor

Exist Prob

Wild/Scenic Protection

Wild/Scenic Tributary or Upstream/Downstream Wild/Scenic Location Cultural value

Fish Presence Value

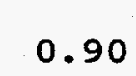

Wildlife Value Threatened/Endangered Fish

AK

Geologic Value

Historic Value

other Value

Recreation value

Scenic Value

$\begin{array}{lll} & 0.90 & \begin{array}{l}\text { Wildife Value } \\ \text { Threatened/Endangered Fish } \\ \text { Threatened/Endangered Wildlife }\end{array} \\ & & \\ & 0.90 & \text { Federal Land Code } 103 \\ & 0.90 & \text { Federal Land Code } 104 \\ \text { Y } & 0.25 & \text { Federal Land Code } 105 \\ & 0.90 & \text { Federal Land Code } 106 \\ & 0.90 & \text { Federal Land Code } 107 \\ & 0.90 & \text { Federal Land Code } 108 \\ \text { Y } & 0.25 & \text { Federal Land Code } 198 \\ \text { Y } & 0.50 & \end{array}$

0.90

0.90

0.90

0.90

0.50

0.90

0.90

0.90

0.90

0.90 


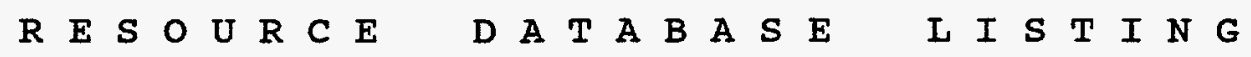

DATE: $10 / 31 / 97$

PAGE NO: 17

FERC

Number

Plant Name

stream

state

07114A DEVIL CANYON

SUSITNA R

Name

county Name

River Basin

MATANUSKA-SUSITNA DIV

SUSITNA RIVER BASIN

Class Owner Name

M ALASKA ENERGY AUTH

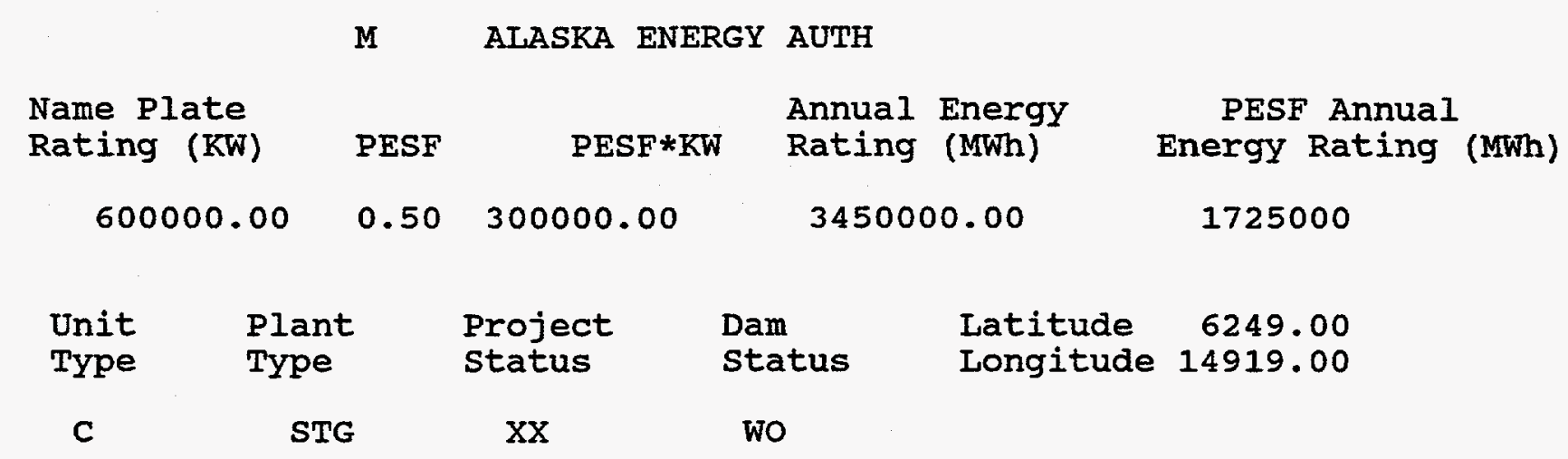

Factor

Exist Prob

Wild/Scenic Protection

Wild/Scenic Tributary or

Upstream/Downstream

wild/scenic Location

Cultural Value

Fish Presence Value

Geologic Value

Historic Value

other Value

Recreation value

Scenic Value
Factor

Exist Prob

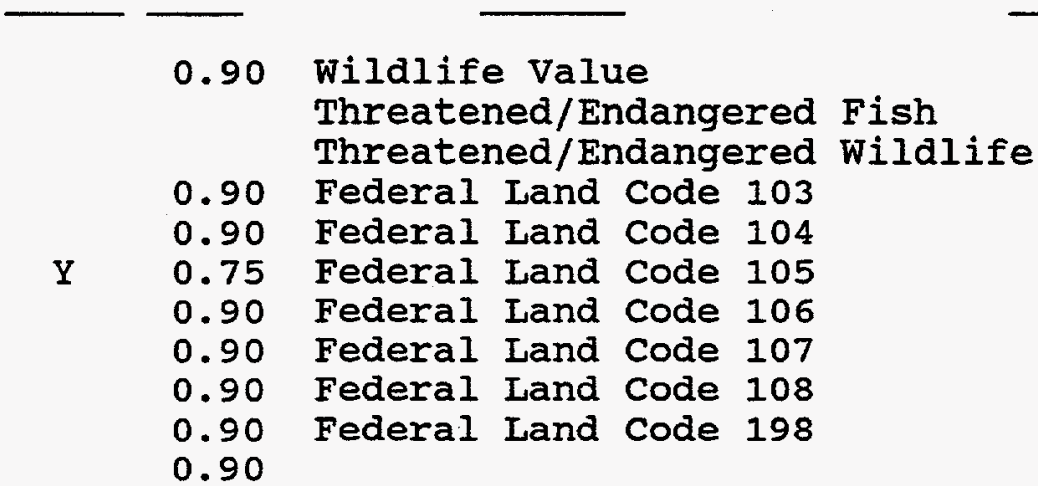

AK 
DATE: $10 / 31 / 97$

FERC

Number

Plant Name

07114B WATANA

County Name

MATANUSKA-SUSITNA DIV
PAGE NO: 18

State

Name

SUSITNA R

$\mathrm{AK}$
River Basin

SUSITNA RIVER BASIN

Class Owner Name

M ALASKA ENERGY AUTH

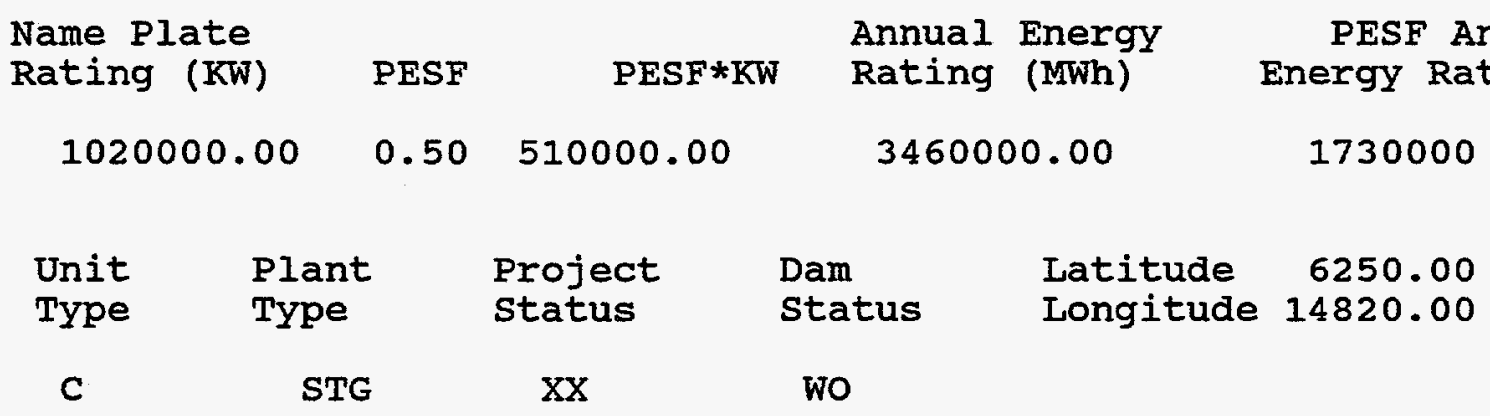

Factor

Exist Prob

Factor

Exist Prob

wild/Scenic Protection

wild/Scenic Tributary or

0.90 Wildlife Value

Threatened/Endangered Fish

Upstream/Downstream

wild/Scenic Location Threatened/Endangered Wildlife

Cultural Value

Fish Presence Value

Geologic Value

Historic Value

other Value

Recreation value

$\begin{array}{ll}0.90 & \text { Federal Land Code } 103 \\ 0.90 & \text { Federal Land Code } 104\end{array}$

Y $\quad 0.75$ Federal Land Code 105

Federal Land Code 106

0.90 Federal Land Code 108

0.90 Federal Land Code 198

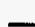

0.90
0.90
0.90
0.90
0.75
0.90
0.90
0.90
0.90
0.90


DATE: $10 / 31 / 97$

PAGE NO: 19

FERC

Number

Plant Name

Stream

State

07633 GRANT LAKE

UP TRAII IK(GRANT LK), TRAIL R

Name

County Name

River Basin

SEWARD DIVISION

KENAI PENINSULA RIVER BAS

$\begin{array}{cr}\text { Class } & \text { Owner Name } \\ \text { R } & \text { KENAI HYDRO INC }\end{array}$

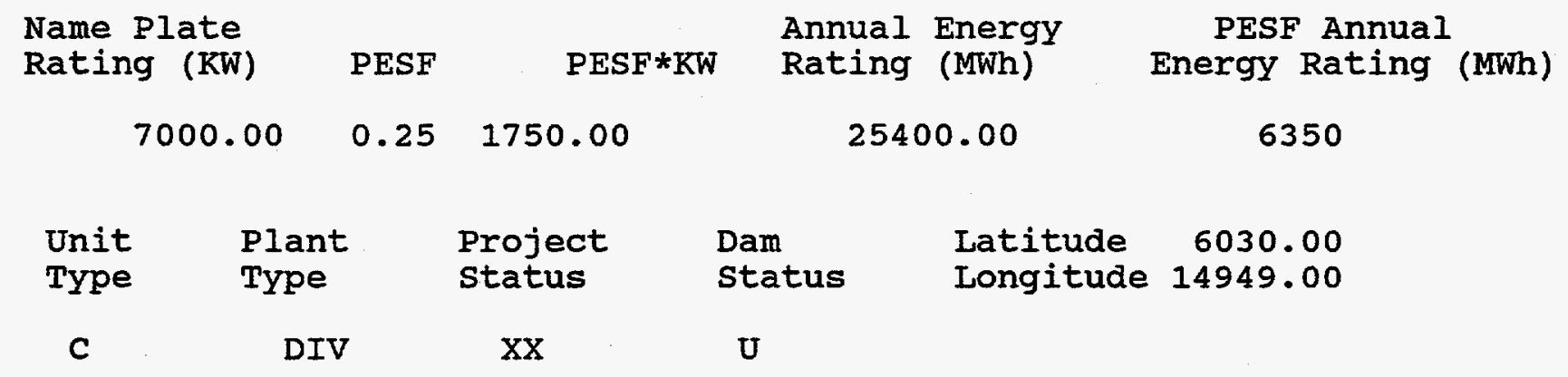

Factor

Exist Prob

Wild/Scenic Protection

wild/Scenic Tributary or

Upstream/Downstream

wild/Scenic Location

Cultural Value

Fish Presence Value

Geologic Value

Historic Value

Other Value

Recreation Value

Scenic Value

0.90
Factor

Exist Prob

0.90 wildlife Value

Threatened/Endangered Fish Threatened/Endangered wildlife

0.90 Federal Land Code 103

0.90 Federal Land Code 104

$\mathrm{Y} \quad 0.25$

Federal Land Code 105

0.90 Federal Land Code 106

0.90 Federal Land code 107

0.90 Federal Land Code 108

0.90 Federal Land Code 198

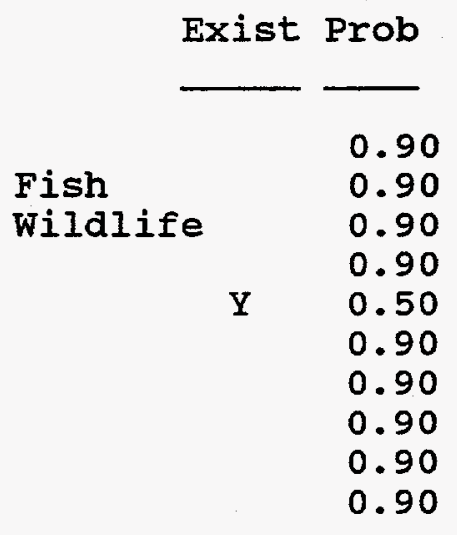

0.90

0.90

0.90

0.50

0.90

.90

0.90
AK 
DATE: $10 / 31 / 97$

FERC

Number

07796

REGIONAL

County Name

YUKON-KOYUKUK DIVISION
PAGE NO: 20

State Name

MINOOK CR, YUKON R

AK

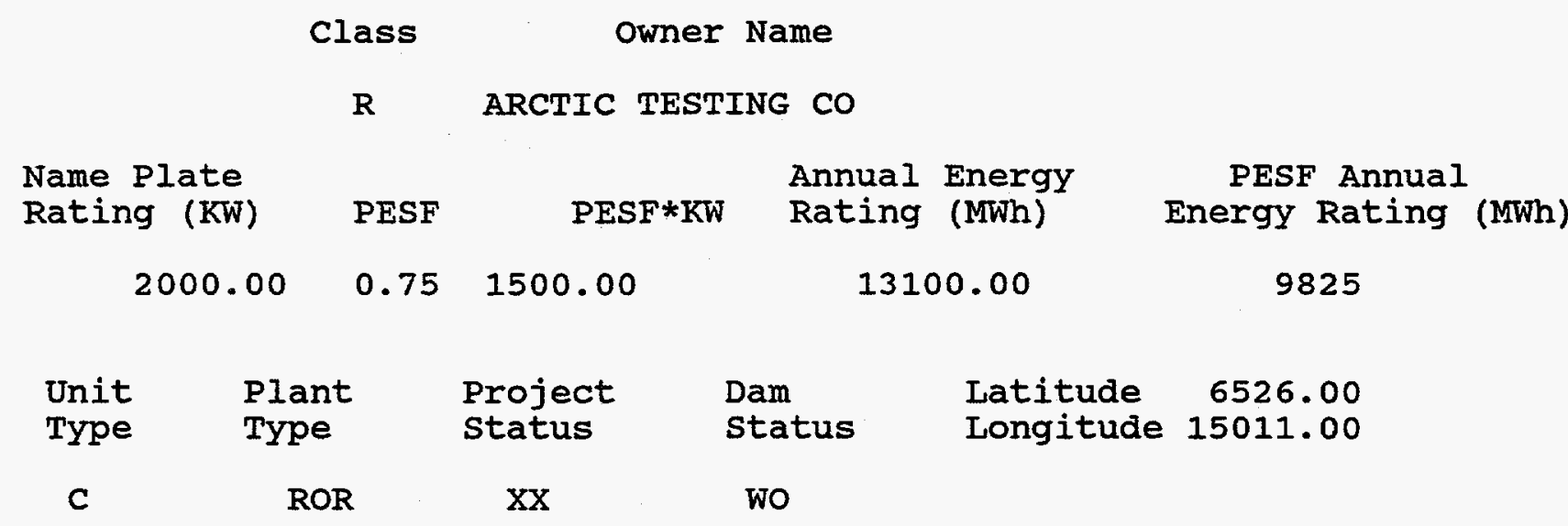

Name Plate Rating (KW) PESF

Annual Energy River Basin

YUKON RIVER BASIN

Factor

Exist Prob

Factor

Exist Prob

Wild/Scenic Protection

wild/Scenic Tributary or Upstream/Downstream wild/Scenic Location Cultural Value Fish Presence Value Geologic Value Historic Value other Value Recreation Value Scenic Value

\section{Exist Prob}

0.90

wilalife value Threatened/Endangered Fish Threatened/Endangered Wildlife

0.90 Federal Land Code 103

0.90 Federal Land Code 104

$\mathrm{Y}$

0.75

0.90

Federal Land Code 105

0.90

0.90

Federal Land Code 106

Federal Land Code 107

0.90

Federal Land Code 108

0.90
0.90

0.90

0.90

0.90

0.90

0.90

0.90

0.90

0.90

0.90 
DATE: $10 / 31 / 97$

FERC

Number

Plant Name

07797 RAMPARTS

county Name

YUKON-KOYUKUK DIVISION

Class

Owner Name

R ARCTIC TESTING CO

Name Plate

Rating (KW)

PESF

Annual Energy

$\mathrm{PESF} * \mathrm{KW}$

Rating (MWh)

22300.00

$0.25 \quad 750.00$

3000.00
River Basin

YUKON RIVER BASIN

PAGE NO: 21

State

Name

AR

\begin{tabular}{|c|c|c|c|c|}
\hline $\begin{array}{l}\text { Unit } \\
\text { Type }\end{array}$ & $\begin{array}{l}\text { Plant } \\
\text { Type }\end{array}$ & $\begin{array}{l}\text { Project } \\
\text { status }\end{array}$ & $\begin{array}{l}\text { Dam } \\
\text { status }\end{array}$ & $\begin{array}{lr}\text { Latitude } & 6528.00 \\
\text { Longitude } & 15012.00\end{array}$ \\
\hline C & ROR & $X X$ & U & \\
\hline
\end{tabular}

Factor

wild/Scenic Protection

wild/Scenic Tributary or

Upstream/ Downstream

wild/Scenic Location

Cultural value

Fish Presence Value

Geologic Value

Historic Value

other Value

Recreation value

Scenic Value
Exist Prob

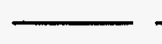

PESF Annual Energy Rating (MWh)

5575
Factor

0.90 Wildlife Value

Threatened/Endangered Fish

Threatened/Endangered Wildlife

0.90 Federal Land Code 103

0.90 Federal Land Code 104

0.25 Federal Land Code 105

0.90 Federal Land Code 106

0.90 Federal Land Code 107

0.90 Federal Land Code 108

0.90 Federal Land Code 198
Exist Prob

0.90

0.90

0.90

0.90

0.90

0.90

0.90

0.90

0.90

0.90 
DATE: $10 / 31 / 97$

PAGE NO: 22

FERC

Number

07798

Plant Name

CANYON CREEK

County Name

YUKON-KOYUKUK DIVISION

YUKON RIVER BASIN

State

Name

AK

\section{Class Owner Name}

R ARCTIC TESTING CO

\begin{tabular}{|c|c|c|c|}
\hline $\begin{array}{l}\text { Name Plate } \\
\text { Rating (KW) }\end{array}$ & PESF & PESF*KW & $\begin{array}{l}\text { Annual Energy } \\
\text { Rating (MWh) }\end{array}$ \\
\hline & & 125.00 & 3650.00 \\
\hline
\end{tabular}

Unit Plant

Type Type

C

ROR

\section{Project status}

$\mathrm{Xx}$
Dam
status

U
PESF Annual Energy Rating (MWh)
Factor

Wild/Scenic Protection wild/Scenic Tributary or Upstream/ Downstream wild/Scenic Location Cultural value Fish Presence value Geologic Value Historic Value other Value Recreation value Scenic Value
Exist Prob

$\begin{array}{lll} & 0.90 & \begin{array}{l}\text { Wildife Value } \\ \text { Threatened/Endangered Fish } \\ \end{array} \\ & & \text { Threatened/Endangered Wildife } \\ 0.90 & \text { Federal Land Code } 103 \\ 0.90 & \text { Federal Land Code } 104 \\ & 0.25 & \text { Federal Land Code } 105 \\ 0.90 & \text { Federal Land Code } 106 \\ 0.90 & \text { Federal Land Code } 107 \\ 0.90 & \text { Federal Land Code } 108 \\ 0.90 & \text { Federal Land Code } 198 \\ 0.90 & \end{array}$

Exist Prob

0.90

0.90

0.90

0.90

0.90

0.90

0.90

0.90

0.90

0.90 
DATE: $10 / 31 / 97$

FERC

Number

Plant Name

07799

TANANA

County Name

YUKON-KOYUKUK DIVISION

\section{Class}

$\mathbf{R}$

ARCTIC TESTING CO

Name Plate

Rating (KW)

1000.00

PESF

PESF*KW

Annual Energy Rating (MWh)

PAGE NO: 23

stream

state

Name

BEAR CR, YUKON R

River Basin

YUKON RIVER BASIN

AK

4500.00

3375
PESF Annual Energy Rating (MWh)

\section{Unit Plant}

Type

Type

C

ROR

$\begin{array}{llr}\text { Dam } & \text { Latitude } & 6518.00 \\ \text { Status } & \text { Longitude } & 15200.00\end{array}$

Project status

Longitude 15200.00

wo

\section{Factor}

Wild/Scenic Protection

wild/Scenic Tributary or Upstream/Downstream wild/Scenic Location Cultural value

Fish Presence Value Geologic Value

Historic Value

other Value

Recreation value

Scenic Value
Exist Prob

$\longrightarrow$

0.90

Wildlife Value

Threatened/Endangered Fish

Threatened/Endangered Wildlife

0.90 Federal Land Code 103

0.90 Federal Land Code 104

$\mathrm{Y} \quad 0.75$

0.90

0.90

0.90

0.90

0.90
Federal Land Code 105

Federal Land Code 106

Federal Land Code 107

Federal Land Code 108

Federal Land Code 198
Exist Prob

0.90

0.90

0.90

0.90

0.90

0.90

0.90

0.90

0.90

0.90 
R E S O U R C E D A T A B A S E L I S T I N G

DATE: $10 / 31 / 97$

PAGE NO: 24

FERC

Number

Plant Name

stream

State

08756 JUNEAU CREEK

JUNEAU CR, KENAI $R$

Name

AK

County Name

River Basin

SEWARD DIVISION

KENAI PENINSULA RIVER BAS

Class Owner Name

R ENERGY STREAM FUND NO 14, LTD

Name Plate

PESF

Annual Energy

Rating (KW)

0.50900 .00

PESF*KW

Rating (MWh)

PESF Annual

1800.00

11800.00

Energy Rating (MWh)

Unit Plant

Type Type

C

ROR

\section{Project} status

$\mathrm{XX}$

Exist Prob

Factor

wild/Scenic Protection

wild/Scenic Tributary or Upstream/Downstream wild/Scenic Location

Cultural value

Fish Presence Value

Geologic Value

Historic Value

other Value

Recreation value

Scenic Value

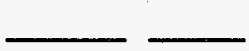

0.90

Wildlife Value

Threatened/Endangered Fish

Threatened/Endangered Wildlife

0.90 Federal Land Code 103

0.90 Federal Land Code 104

$Y \quad 0.75$ Federal Land Code 105

0.90 Federal Land Code 106

0.90 Federal Land Code 107

0.90 Federal Land Code 108

$Y \quad 0.75$ Federal Land Code 198
5900

6029.00

14948.00 
DATE: $10 / 31 / 97$

FERC

Number

08765

BEAR CREEK

County Name

SEWARD DIVISION

Class

R ENERGY STREAM FUND NO 16, LTD

Name Plate

Rating (KW)

PESF

Annual Energy

PESF*KW

BEAR CR, TURNAGAIN ARM

$$
\text { River Basin }
$$

KENAI PENINSULA RIVER BAS

PAGE NO: 25

State

Name

AK

$$
250.00 \quad 0.50 \quad 125.00
$$

Unit Plant

Type Type

C

ROR

\section{Project} status

$\mathrm{XX}$
Factor

Wild/Scenic Protection wild/Scenic Tributary or Upstream/Downstream wild/Scenic Location Cultural value Fish Presence Value Geologic Value Historic Value other Value Recreation value Scenic Value
Exist Prob

Factor

Latitude Longitude 14930.00

$\begin{array}{llr}\text { Dam } & \text { Latitude } & 6020.00 \\ \text { Status } & \text { Longitude } & 14930.00\end{array}$

wo
PESF Annual Energy Rating (MWh)

$\begin{array}{lll}0.90 & \begin{array}{l}\text { Wildife Value } \\ \text { Threatened/Endangered Fish } \\ \text { Threatened/Endangered Wildlife }\end{array} \\ & 0.90 & \text { Federal Land Code } 103 \\ 0.90 & \text { Federal Land Code } 104 \\ \text { Y } & 0.75 & \text { Federal Land Code } 105 \\ 0.90 & \text { Federal Land Code } 106 \\ 0.90 & \text { Federal Land Code } 107 \\ 0.90 & \text { Federal Land Code } 108 \\ 0.90 & \text { Federal Land Code } 198 \\ 0.90 & \end{array}$

Exist Prob

500 
DATE: $10 / 31 / 97$

PAGE NO: 26

FERC

Number

Plant Name

Stream

State

08766

STEEP CREEK

STEEP CR, EYAK R

Name

county Name

River Basin

CORDOVA-MCCARTHY DIV

KENAI PENINSULA RIVER BAS

Class Owner Name

R ENERGY STREAM FUND NO 3,LTD

Name Plate

PESF

Annual Energy

Rating ( $\mathrm{KW})$

PESF * KW

Rating (MWh)

PESF Annual

870.00

$0.75 \quad 652.50$

3800.00

Energy Rating (MWh)

2850

\begin{tabular}{|c|c|c|c|c|}
\hline $\begin{array}{l}\text { Unit } \\
\text { Type }\end{array}$ & $\begin{array}{l}\text { Plant } \\
\text { Type }\end{array}$ & $\begin{array}{l}\text { Project } \\
\text { Status }\end{array}$ & $\begin{array}{l}\text { Dam } \\
\text { status }\end{array}$ & $\begin{array}{lr}\text { Latitude } 6032.00 \\
\text { Longitude } 14535.00\end{array}$ \\
\hline C & ROR & $x x$ & wo & \\
\hline
\end{tabular}

Factor

Exist Prob

Factor

Exist Prob

Wild/Scenic Protection

wild/Scenic Tributary or

0.90 Wildlife Value

0.90

Upstream/Downstream

Threatened/Endangered Fish

0.90

wild/Scenic Location

Threatened/Endangered Wildlife

0.90

0.90 Federal Land Code 103

0.90

Cultural Value

0.90 Federal Land Code 104

0.90 Federal Land Code 105

$\mathrm{Y}$

0.75

0.90 Federal Land Code 106

0.90

Geologic Value

0.90 Federal Land Code 107

0.90

Historic Value

0.90 Federal Land Code 108

0.90

other.Value

Recreation value

0.90

Scenic Value

0.90

Federal Land Code 198

0.90

0.90 


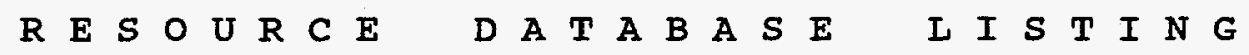

DATE: $10 / 31 / 97$

PAGE NO: 27

FERC

Number

Plant Name

Stream

state

08767 BEARTRAIL

BEARTRAIL CR, IBECK CR

Name

County Name

River Basin

CORDOVA-MCCARTHY DIV

COPPER RIVER BASIN

Class Owner Name

R ENERGY STREAM FUND NO 7,LTD

Name Plate

$\begin{array}{lll}\text { PESF } & \text { PESF*KW } & \text { Annual Energy } \\ \text { Rating (MWh) }\end{array}$

PESF Annual

Rating ( $\mathrm{KW}$ )

$0.50 \quad 425.00$

3300.00

Energy Rating (MWh)

850.00

0.50

Unit Plant

Type Type

C

ROR

Factor

Wild/Scenic Protection

Wild/Scenic Tributary or

Upstream/Downstream

wild/Scenic Location

Cultural Value

Fish Presence Value

Geologic Value

Historic Value

other value

Recreation value

Scenic Value
Exist Prob

0.90

Threatened/Endangered Fish
Threatened/Endangered Wildife

0.90 Federal Land code 103

0.90 Federal Land Code 104

Y $\quad 0.75$ Federal Land Code 105

0.90 Federal Land Code 106

0.90 Federal Land Code 107

0.90 Federal Land Code 108

0.90 Federal Land Code 198
Dam

Status

Latitude $\quad 6227.00$

Longitude 14508.00

AR

1650

0.90

Exist Prob

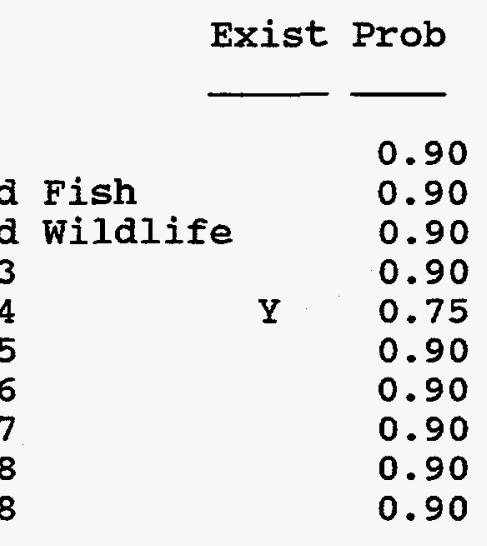


R E S O U R C E

DATE: $10 / 31 / 97$
D A T A B A S E

\begin{abstract}
C A $A$ B $A S$
\end{abstract}
FERC

Number

Plant Name

08768 LOST CREEK

County Name

SEWARD DIVISION

Class Owner Name

R ENERGY STREAM FUND NO 15, LTD

Name Plate

Rating (KW)

PESF

PESF*KW

Annual Energy Rating (MWh)

$0.75 \quad 675.00$

900.00

675.00

Unit Plant

Type Type

C

ROR

Exist Prob

Factor

Latitude 6003.00

status

Dam

Status

Longitude 14928.00

wo
State

Name

AK
PESF Annual Energy Rating (MWh)

2925

\author{
Factor \\ Wild/Scenic Protection \\ wild/Scenic Tributary or \\ Upstream/Downstream \\ wild/Scenic Location \\ Cultural Value \\ Fish Presence Value \\ Geologic Value \\ Historic Value \\ other value \\ Recreation value \\ Scenic Value
}

Exist Prob

0.90 Wildlife Value

Threatened/Endangered Fish

Threatened/Endangered Wildlife

0.90 Federal Land Code 103

0.90 Federal Land Code 104

Y 0.75 Federal Land Code 105

0.90 Federal Land Code 106

0.90 Federal Land Code 107

0.90 Federal Land Code 108

0.90 Federal Land code 198
Exist Prob

0.90

0.90

0.90

0.90

0.90

0.90

0.90

0.90

0.90

0.90

0.90 


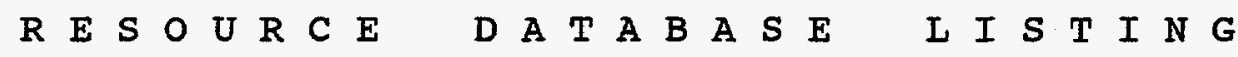

DATE: $10 / 31 / 97$

PAGE NO: 29

FERC

Number

Plant Name

08769 KASIDAYA CREEK

County Name

SKAGWAY-YAKUTAT DIV

Stream

State

Name

KASIDAYA CREEK, TAIYA INLET

$A K$

River Basin

JUNEAU GROUP

Class Owner Name

R ENERGY STREAM FUND NO 12 , LTD

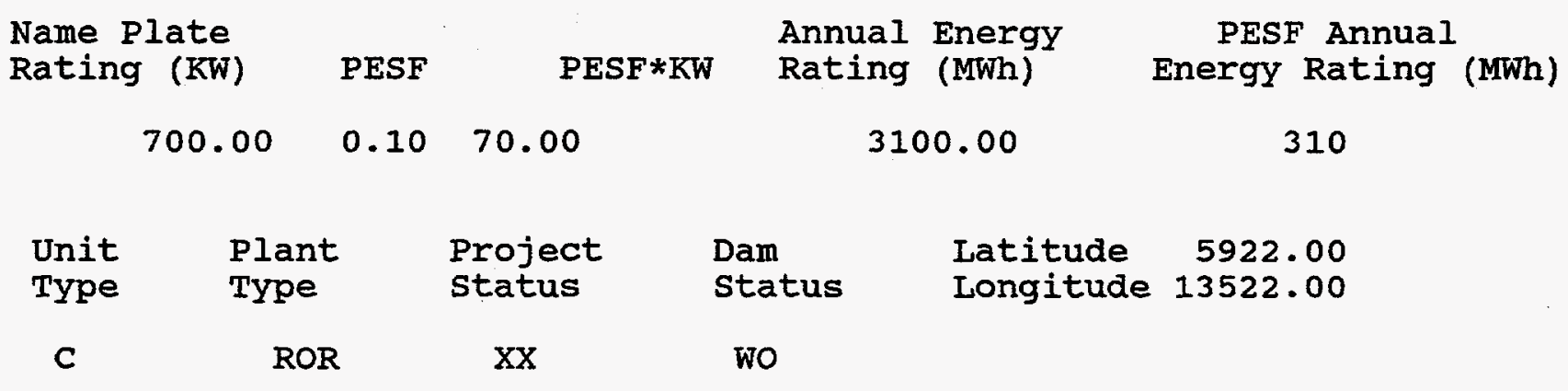

Factor

wild/Scenic Protection

wild/Scenic Tributary or Upstream/ Downstream wild/Scenic Location

Cultural value

Fish Presence Value

Geologic Value

Historic Value

other Value

Recreation Value

Scenic Value
Exist Prob

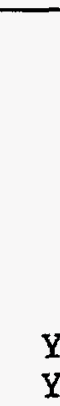

Factor

Exist Prob

0.90 Wildlife Value Threatened/Endangered Fish Threatened/Endangered Wildlife Federal Land Code 103 Federal Land Code 104 Federal Land Code 105 Federal Iand Code 106 Federal Land code 107 Federal Land Code 108 Federal Land Code 198
0.90

0.90

0.90

0.10

0.90

0.90

0.90

0.90

0.90

0.90

0.90 
R E S O U R C E

DATE: $10 / 31 / 97$

FERC

Number

Plant Name

08770 P C 2

county Name

CORDOVA-MCCARTHY DIV
D A T A B A S E

L I S T I N G

PAGE NO: 30

Class Owner Name

M CORDOVA PUBLIC UTILITIES

Name Plate

Rating (KW)

PESF

Annual Energy

PESF*KW

Rating (MWh)

PESF Annual

2000.00

0.10200 .00

13000.00

Unit Plant

Project

Dam

Type

Type

status

Status

Latitude

6037.00

C

ROR

$\mathrm{XX}$

$\mathbf{U}$

Factor

Wild/scenic Protection

wild/scenic Tributary or Upstream/Downstream wild/Scenic Location

Cultural value

Fish Presence Value

Geologic Value

Historic Value

other Value

Recreation Value

Scenic Value
Exist Prob

Factor

Exist Prob

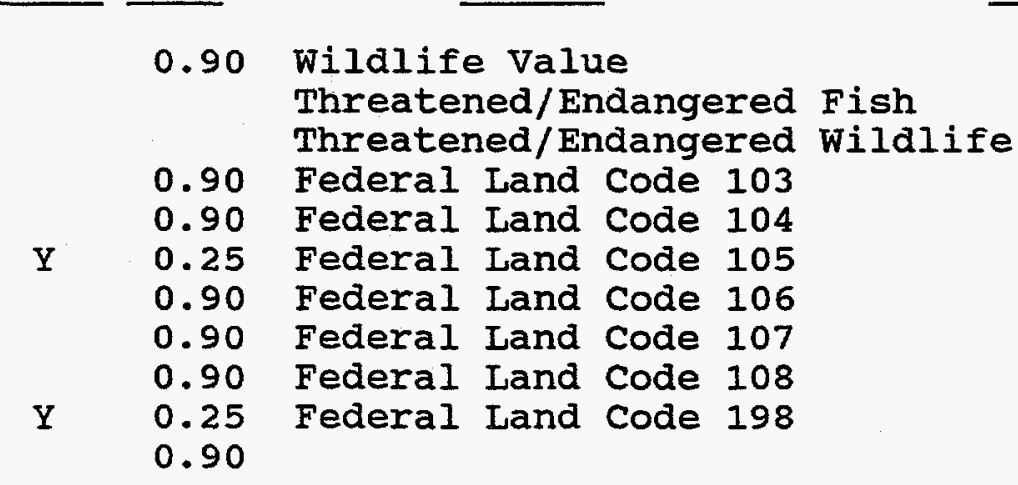

State

Name

AK
1300

Energy Rating (MWh) 
DATE: $10 / 31 / 97$

FERC

Number

Plant Name

08772 GRANT LAKE

County Name

SEWARD DIVISION

Class Owner Name

R ENERGY STREAM FUND NO 13, LTD

Name Plate

Rating ( $\mathrm{KW})$

PESF

Annual Energy

830.00

0.25207 .50

5100.00

PAGE NO: 31

GRANT CR, TRAIL R

River Basin

KENAI PENINSULA RIVER BAS
State

Name

AK

\begin{tabular}{|c|c|c|c|c|}
\hline $\begin{array}{l}\text { Unit } \\
\text { Type }\end{array}$ & $\begin{array}{l}\text { Plant } \\
\text { Type }\end{array}$ & $\begin{array}{l}\text { Project } \\
\text { status }\end{array}$ & $\begin{array}{l}\text { Dam } \\
\text { status }\end{array}$ & $\begin{array}{lr}\text { Latitude } & 6029.00 \\
\text { Longitude } & 14946.00\end{array}$ \\
\hline C & ROR & $X X$ & $\mathrm{U}$ & \\
\hline
\end{tabular}

Factor

Wild/Scenic Protection

Wild/Scenic Tributary or

Upstream/Downstream

wild/Scenic Location

Cultural value

Fish Presence Value

Geologic Value

Historic Value

other Value

Recreation value

Scenic Value
Exist Prob

Factor

Exist Prob

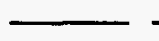

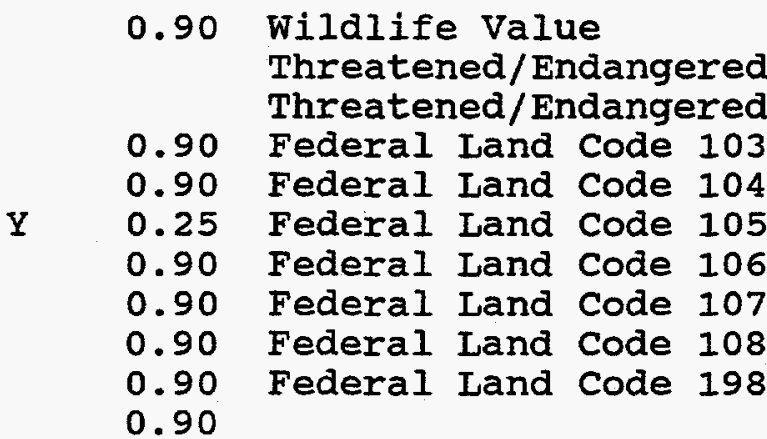

PESF Annual Energy Rating (MWh)

1275 
DATE: $10 / 31 / 97$

PAGE NO: 32

FERC

Number

Plant Name

Stream

State

08827 SHIP CREEK

CITY WATER \& WASTE WTR PIPELIN

Name

County Name

River Basin

MATANUSKA-SUSITNA DIV

KENAI PENINSULA RIVER BAS

\section{Class Owner Name}

M ANCHORAGE, CITY OF

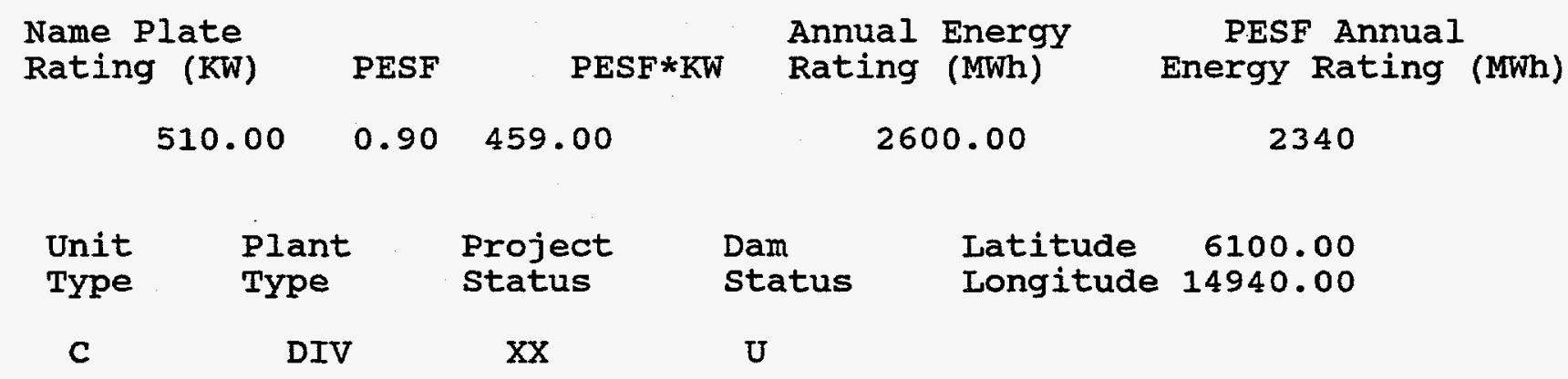

Factor

Wild/Scenic Protection

Wild/Scenic Tributary or Upstream/Downstream wild/Scenic Location Cultural value

Fish Presence Value

Geologic Value

Historic Value

other Value

Recreation value

Scenic Value
Exist Prob

0.90

Wildlife value

Threatened/Endangered Fish

Threatened/Endangered Wildlife

0.90 Federal Land Code 103

0.90 Federal Land Code 104

0.90 Federal Land Code 105

0.90 Federal Land Code 106

0.90 Federal Land Code 107

0.90 Federal Land Code 108

0.90 Federal Land Code 198

0.90
Exist Prob

0.90

0.90

0.90

0.90

0.90

0.90

0.90

0.90

0.90

0.90 
DATE: $10 / 31 / 97$

FERC

Number

Plant Name

09760

TREADWELL DITCH

County Name

JUNEAU DIVISION

Class

Name Plate

Rating (KW)

2200.00
PAGE NO: 33

state

Name
$\mathbf{R}$

PESF

RE-ENERTEK

Owner Name

JUNEAU GROUP
TREADWELI DH, GASTINEAU CHANNEL AR

River Basin

$0.90 \quad 1980.00$

Annual Energy

Rating (MWh)

PESF Annual Energy Rating (MWh)

7920

$\begin{array}{cccll}\text { Unit } & \text { Plant } & \text { Project } & \text { Dam } & \text { Latitude } \\ \text { Type } & \text { Type } & \text { Status } & \text { Status } & \text { Longitude } 13419.00 \\ \text { C } & \text { ROR } & \text { XX } & \text { wo } & \end{array}$

Factor

Exist Prob

Factor

Exist Prob

Wild/Scenic Protection

wild/Scenic Tributary or

0.90 Wildlife Value

0.90

Threatened/Endangered Fish

0.90

Upstream/Downstream Threatened/Endangered Wildlife

0.90

0.90 Federal Land Code 103

0.90

0.90 Federal Land Code 104

0.90

0.90 Federal Land Code 105

0.90

0.90 Federal Land Code 106

0.90

0.90 Federal Land Code 107

0.90

0.90 Federal Land Code 108

0.90

other value

Recreation Value

0.90

Federal Land Code 198

0.90

Scenic Value

0.90 


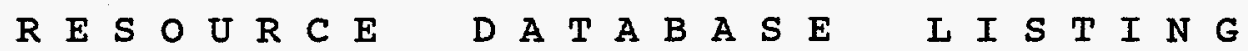

DATE: $10 / 31 / 97$

PAGE NO: 34

FERC

Number

Plant Name

Stream

state

09763 NUGGET CREEK

NUGGET CR, MENDENHAL LK

Name

county Name

River Basin

JUNEAU DIVISION

JUNEAU GROUP

Class Owner Name

R RE-ENERTEK INC

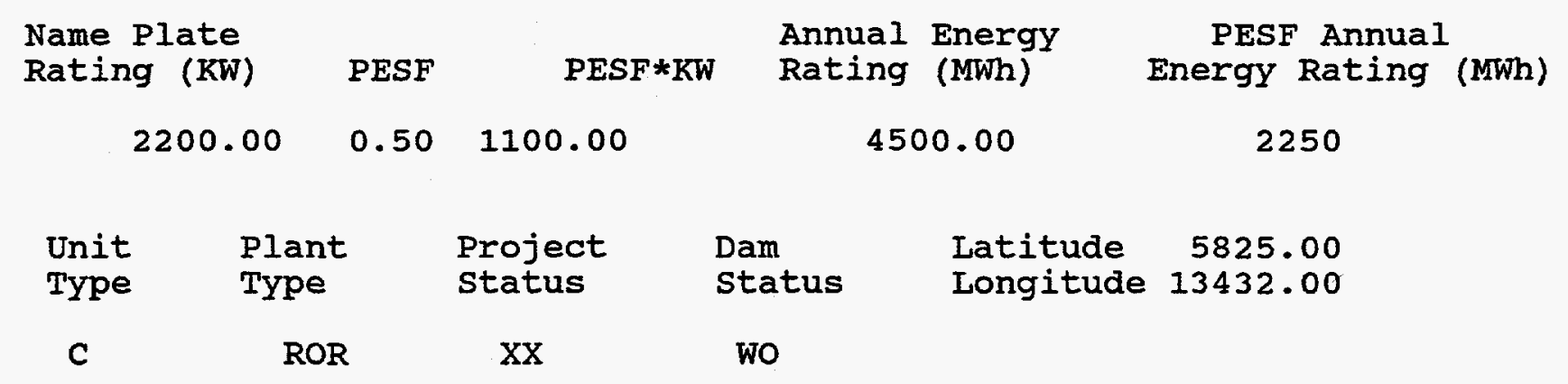

Factor

Wild/Scenic Protection

Wild/Scenic Tributary or Upstream/Downstream wild/Scenic Location Cultural value

Fish Presence Value

Geologic Value

Historic Value

other Value

Recreation value

Scenic Value
Exist Prob

0.90

Wildlife Value

Threatened/Endangered Fish Threatened/Endangered Wildlife

0.90 Federal Land Code 103

0.90 Federal Land Code 104

0.75 Federal Land Code 105

0.90 Federal Land Code 106

0.90 Federal Land Code 107

0.90 Federal Land Code 108

0.90 Federal Land code 198
Exist Prob

0.90

0.90

0.90

0.90

0.75

0.90

0.90

0.90

0.90

0.90 
DATE: $10 / 31 / 97$

PAGE NO: 35

FERC

Number

Plant Name

Stream

State

09996 REDISCOVERY LODGE

BELI CR

Name

AK

County Name

River Basin

OUTER KETCHIKAN DIV

KETCHIKAN GROUP

Class Owner Name

R NW DISCOVERY JT VENTURE

Name Plate

Rating (KW)

PESF

Annual Energy

PESF Annual

115.00

0.25

28.75

200.00

Energy Rating (MWh)

Unit Plant

Type Type

Project

C

ROR

Exist Prob

Factor

wild/Scenic Protection

Wild/Scenic Tributary or Upstream/ Downstream wild/Scenic Location Cultural value

Fish Presence Value Geologic Value

Historic Value

other Value

Recreation value

Scenic Value status

LE

-

0.90

wildife value

Threatened/Endangered Fish

Threatened/Endangered Wildlife

Latitude 5557.00

Dam
status

Longitude 13129.00

$\mathrm{U}$

Factor

50

Exist Prob

Federal Land Code 103

0.90 Federal Land Code 104

Y $\quad 0.25$ Federal Land Code 105

0.90 Federal Land Code 106

0.90 Federal Land Code 107

0.90 Federal Land Code 108

0.90 Federal Land Code 198

0.90
0.90

0.90

0.90

0.90

0.50

0.90

0.90

0.90

0.90

0.90 
R E S O U R C E

DATE: $10 / 31 / 97$
D A T A B A S E

L I S T I N G
FERC

Number

10064 WHITTIER CREEK

County Name

VALDEZ-CHITINA-WHITT DV
PAGE NO: 36

State

Name

WHITTIER CR, PASSAGE CANAL AK

River Basin

KENAI PENINSULA RIVER BAS

\section{Class Owner Name}

$R$ LATHAN, WILLIAM J

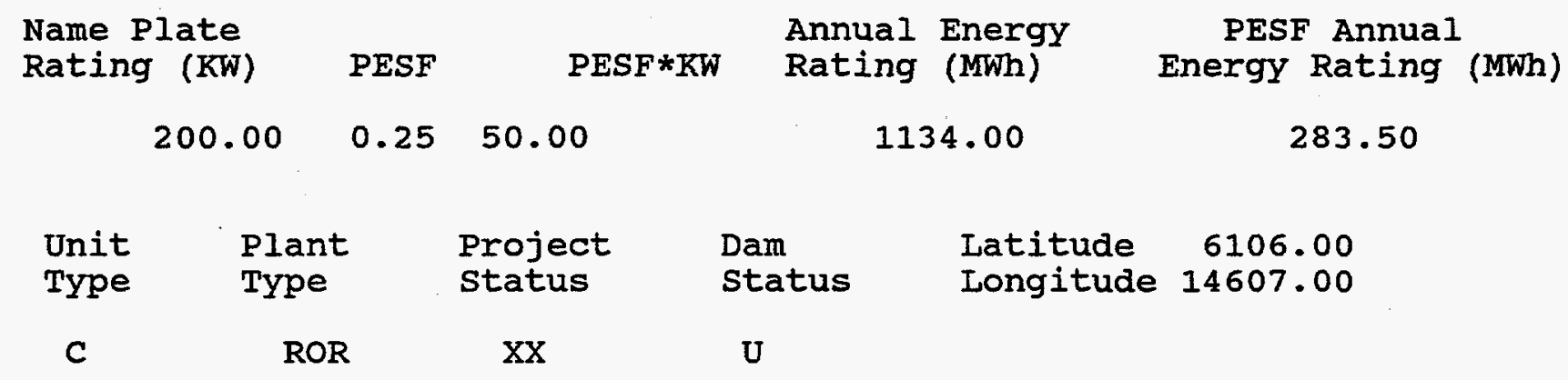

Factor

Exist Prob

Factor

Exist Prob

wild/Scenic Protection

wild/Scenic Tributary or

\subsection{0 wildlife value}

Threatened/Endangered Fish Threatened/Endangered Wildlife

Upstream/Downstream

Wild/Scenic Location

Cultural value

Fish Presence Value

Geologic Value

Historic Value

other Value

Recreation value

Scenic Value
0.90

0.90

0.90

0.90

$\mathrm{Y}$

0.50

0.90

0.90

0.90

0.90

0.90 
DATE: $10 / 31 / 97$

FERC

Number

Plant Name

10524 POWER CREEK

County Name

CORDOVA-MCCARTHY DIV
PAGE NO: 37

State

Name

AR
POWER CR, EYAK LAKE

River Basin

KENAI PENINSULA RIVER BAS

Class Owner Name

$R$ NORTH COAST DEV CO INC

$\begin{array}{ccccc}\begin{array}{l}\text { Name Plate } \\ \text { Rating (KW) }\end{array} & \text { PESF } & \text { PESF*KW } & \begin{array}{c}\text { Annual Energy } \\ \text { Rating } \\ (M W h)\end{array} & \begin{array}{c}\text { PESF Annual } \\ \text { Energy Rating (MWh) }\end{array} \\ 6000.00 & 0.10 & 600.00 & 30000.00 & 3000\end{array}$

$\begin{array}{lllll}\text { Unit } & \text { Plant } & \text { Project } & \text { Dam } & \text { Latitude } 6036.00 \\ \text { Type } & \text { Type } & \text { Status } & \text { Status } & \text { Longitude } 14531.00\end{array}$

C ROR $\quad \mathrm{XX} \quad \mathrm{U}$

Factor

Exist Prob

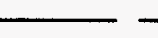

wild/Scenic Protection

Wild/Scenic Tributary or

Upstream/Downstream

wild/Scenic Location

Cultural value

Fish Presence Value

Geologic Value

Historic Value

other Value

Recreation Value

Scenic Value

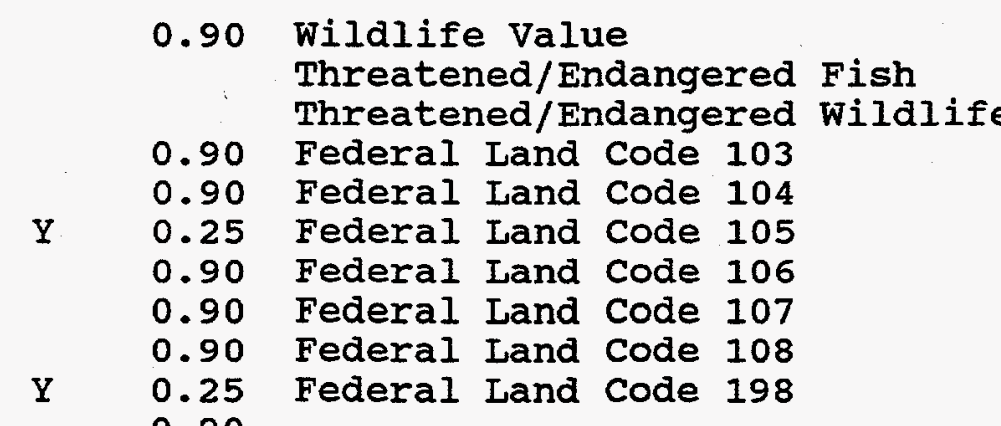

Factor

Exist Prob

0.90
0.90

0.90

0.90

0.90

$Y$
0.90

0.90

0.90

0.90

0.90 
DATE: $10 / 31 / 97$

PAGE NO: 38

FERC

Number

Plant Name

Stream

State

10566 LUTAK INLET

UNN STR, LUTAK INLET

Name

County Name

River Basin

SITKA DIVISION

JUNEAU GROUP

class Owner Name

R SOUTHERN ENERGY, INC

Name Plate

PESF

Annual Energy

Rating (KW)

PESF*KW

Rating (MWh)

PESF Annual

$$
150.00 \quad 0.75 \quad 112.50
$$

519.00

Energy Rating

(MWh)

AK

\begin{tabular}{|c|c|c|c|c|}
\hline $\begin{array}{l}\text { Unit } \\
\text { Type }\end{array}$ & $\begin{array}{l}\text { Plant } \\
\text { Type }\end{array}$ & $\begin{array}{l}\text { Project } \\
\text { status }\end{array}$ & $\begin{array}{l}\text { Dam } \\
\text { status }\end{array}$ & $\begin{array}{lr}\text { Latitude } & 5907.00 \\
\text { Longitude } & 13527.00\end{array}$ \\
\hline C & ROR & $\mathrm{xx}$ & Wo & \\
\hline
\end{tabular}

Factor

Wild/Scenic Protection

Wild/Scenic Tributary or Upstream/Downstream wild/Scenic Location Cultural value

Fish Presence Value

Geologic Value

Historic Value

other Value

Recreation Value

Scenic Value
Exist Prob

0.90

Wildlife Value Threatened/Endangered Fish Threatened/Endangered Wildlife

0.90 Federal Land Code 103

0.90 Federal Land Code 104

$\mathrm{Y}$

Federal Land Code 105

0.90

0.90

Federal Land Code 106

Federal Land Code 107

0.90 Federal Land Code 108

0.90 Federal Land Code 198

0.90

389.25

Exist Prob

0.90

0.90

0.90

0.90

0.90

0.90

0.90

0.90

0.90

0.90 
FERC

Number

10681
Plant Name

SHEEP CREEK

county Name

JUNEAU DIVISION

Class Owner Name

R ECHO BAY EXPLOR, INC

Name Plate

Rating (KW)

PESF

PESF*KW

Annual Energy

3100.00

$0.50 \quad 1550.00$

17000.00
Stream

River Basin

JUNEAU GROUP
SHEEP CR, TAKU INLET
Unit

Type

$$
\begin{aligned}
& \text { Plant } \\
& \text { Type } \\
& \text { ROR }
\end{aligned}
$$

C state

Name

AK
Factor

wild/Scenic Protection

Wild/Scenic Tributary or

Upstream/Downstream

wild/Scenic Location

Cultural value

Fish Presence Value

Geologic Value

Historic Value

other value

Recreation value

Scenic Value

\section{Project} status

$X X$
Exist Prob

Factor

\section{Latitude \\ Longitude 13419.00 \\ status}

wo
PESF Annual Energy Rating (MWh)

8500 
DATE: $10 / 31 / 97$

PAGE NO: 40

FERC

Number

10685A CRATER LAKE

County Name

CORDOVA-MCCARTHY DIV

Plant Name
Class

$R$ NORTH COAST DEV CO INC
River Basin

KENAI PENINSULA RIVER BAS

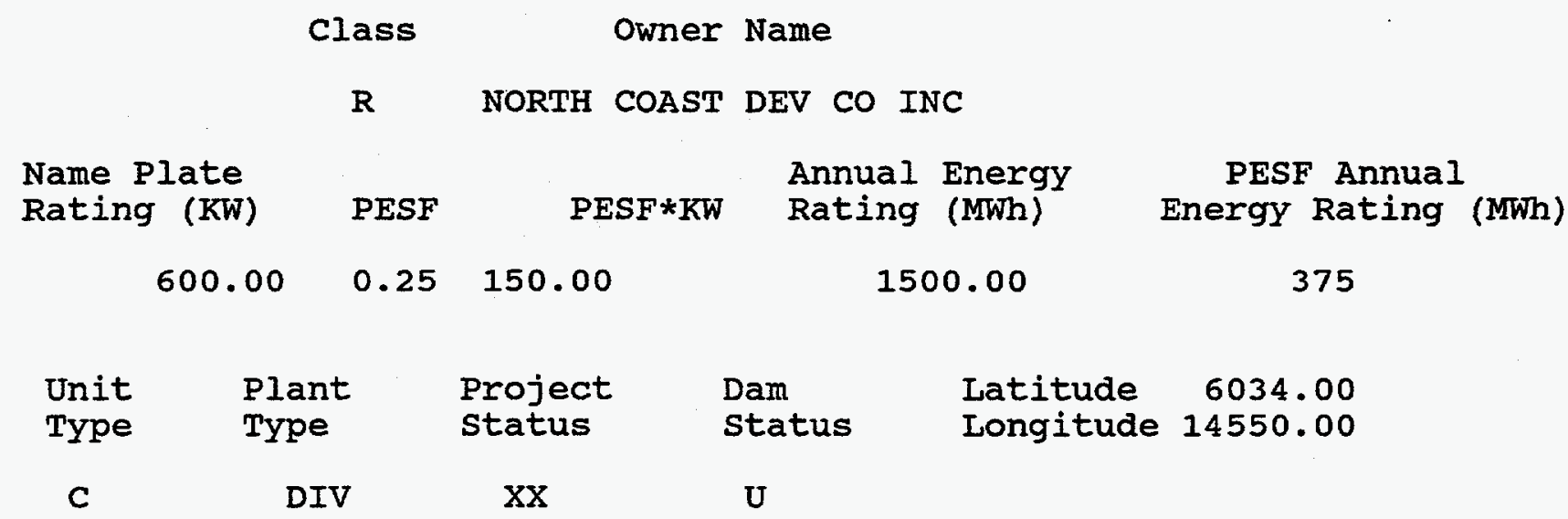

Factor

wild/Scenic Protection Wild/Scenic Tributary or Upstream/Downstream wild/Scenic Location Cultural value Fish Presence Value Geologic Value Historic Value other value Recreation value Scenic Value
Exist Prob

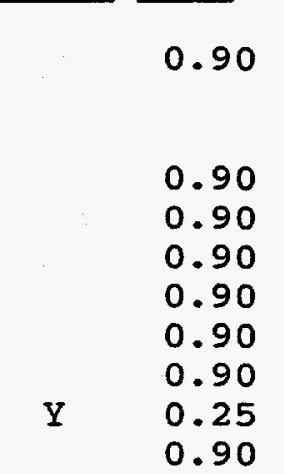

Factor

Exist Prob

Wildlife value

Threatened/Endangered Fish Threatened/Endangered Wildlife

0.90

0.90

0.90

Federal Land Code 103

Federal Land Code 104

Federal Land Code 105

Federal Land Code 106

Federal Land Code 107

Federal Land Code 108

Federal Land Code 198
0.90

0.90

0.90

0.90

0.90

0.90

0.90 
DATE: $10 / 31 / 97$

FERC

Number Plant Name

10685B CRATER LAKE

County Name

CORDOVA-MCCARTHY DIV
PAGE NO: $4 I$

State

Name

AK

\section{Class Owner Name}

R NORTH COAST DEV CO INC

$\begin{array}{lcccc}\begin{array}{l}\text { Name Plate } \\ \text { Rating (KW) }\end{array} & \text { PESF } & \text { PESF*KW } & \begin{array}{c}\text { Annual Energy } \\ \text { Rating (MWh) }\end{array} & \begin{array}{c}\text { PESF Annual } \\ \text { Energy Rating (MWh) }\end{array} \\ 1000.00 & 0.75 & 750.00 & 2500.00 & 1875\end{array}$

$\begin{array}{lllll}\text { Unit } & \text { Plant } & \text { Project } & \text { Dam } & \text { Latitude } 6034.00 \\ \text { Type } & \text { Type } & \text { Status } & \text { Status } & \text { Longitude } 14550.00\end{array}$

$\begin{array}{llll}C & \text { DIV } & \mathrm{XX} & \text { wo }\end{array}$

Factor

Wild/Scenic Protection

Wild/Scenic Tributary or Upstream/Downstream wild/Scenic Location Cultural Value Fish Presence Value Geologic Value Historic Value other value Recreation Value Scenic Value

\section{Exist Prob}

0.90

Wildife Value

Threatened/Endangered Fish Threatened/Endangered Wildlife

0.90 Federal Land Code 103

0.90 Federal Land Code 104

0.90 Federal Land Code 105

0.90 Federal Land Code 106

0.90 Federal Land Code 107

0.90 Federal Land Code 108

$\mathrm{Y}$

0.75

0.90
Exist Prob

0.90

0.90

0.90

0.90

0.90

0.90

0.90

0.90

0.90

0.90 
DATE: $10 / 31 / 97$

PAGE NO: 42

FERC

Number

Plant Name

10686 LAAKE REDFIELD

LAKE REDFIELD, YAKUTAT BAY

State

Name

county Name

River Basin

SKAGWAY-YAKUTAT DIV

COPPER RIVER BASIN

$\begin{array}{cc}\text { Class } & \text { Owner Name } \\ \text { R } & \text { NORTH COAST DEV CO INC }\end{array}$

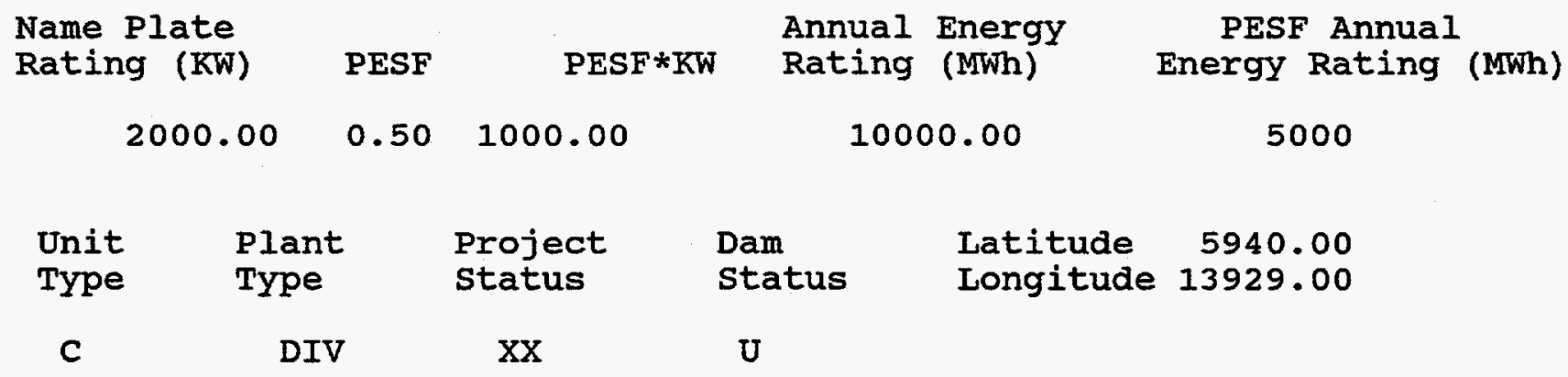

Factor

Exist Prob

Factor

Exist Prob

wild/Scenic Protection

wild/Scenic Tributary or

0.90 Wildlife Value

Threatened/Endangered Fish

AK

Upstream/Downstream

wild/Scenic Location

Cultural value Threatened/Endangered Wildlife

0.90 Federal Land Code 103

0.90 Federal Land Code 104

0.90 Federal Land Code 105

0.90 Federal Land Code 106

0.90 Federal Land Code 107

0.90 Federal Land Code 108

0.90 Federal Land Code 198

-

Geologic Value

Historic Value

0.90

0.90

0.90

0.90

0.90

0.50

0.90

0.90

0.90

0.90

Recreation value

Scenic Value

0.90 

R E S O U R C E
D A T A B A S E
I I S T I N G

DATE: $10 / 31 / 97$

PAGE NO: 43

FERC

Number

Plant Name

Stream

state

10773 BURNETT R HATCHERY

BURNETT $\mathbf{R}$

Name

$\mathrm{AK}$

County Name

River Basin

WRANGEIL-PETERSBURG DIV KETCHIKAN GROUP

Class owner Name

I ALASKA AQUACULTURE INC

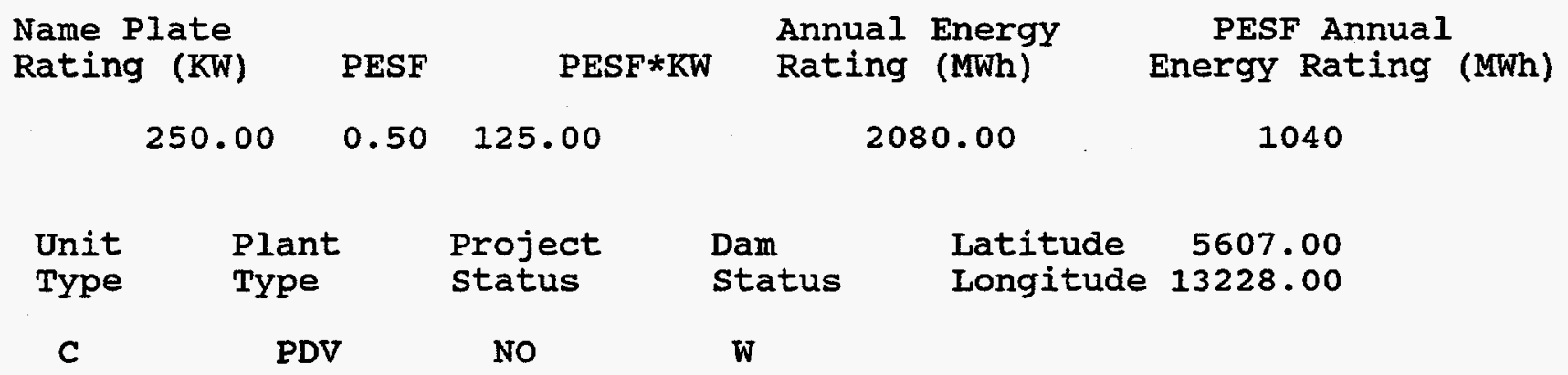

Factor

Exist Prob

Factor

Exist Prob

0.90 Wildlife Value

Threatened/Endangered Fish

Threatened/Endangered wildlife

0.90 Federal Land code 103

0.90 Federal Land Code 104

0.75 Federal Land Code 105

0.90 Federal Land Code 106

0.90 Federal Land code 107

0.90 Federal Land Code 108

0.90 Federal Land code 198

0.90
0.90

0.90

0.90

0.90

0.75

0.90

0.90

0.90

0.90

0.90

Recreation Value

Scenic Value 
R E S O U R C E D A T A B A S E I I S T I N G

DATE: $10 / 31 / 97$

PAGE NO: 44

FERC

Number

Plant Name

Stream

State

Name

11077 GOAT LAKE

SKAGWAY R(GOAT LAKE)

AK

county Name

River Basin

SKAGWAY-YAKUTAT DIV

JUNEAU GROUP

Class Owner Name

$R$ ALASKA PWR \& TELEPHONE CO

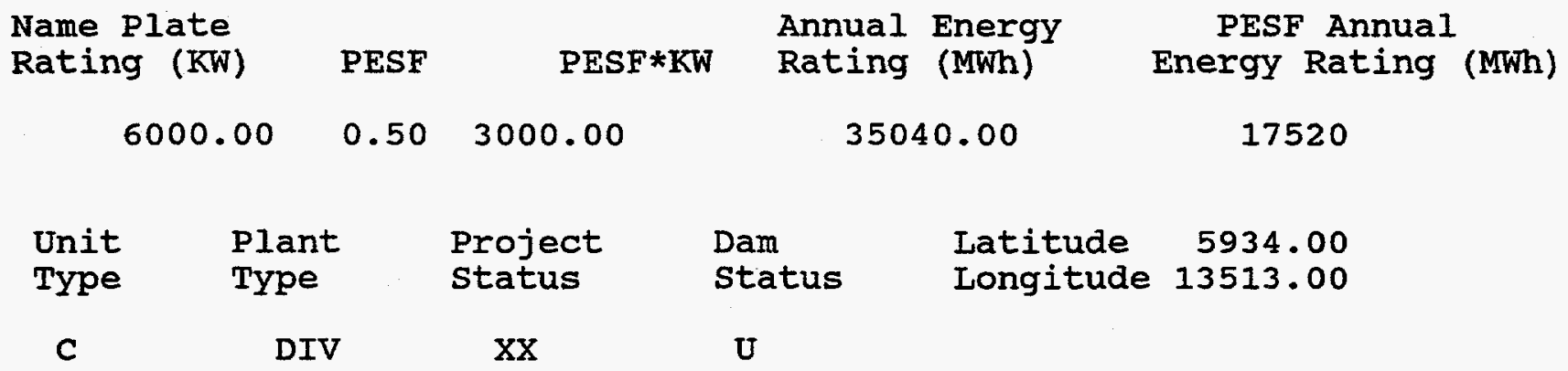

Factor

Exist Prob

Factor

Exist Prob

wild/Scenic Protection

wild/Scenic Tributary or

0.90 Wildlife Value

0.90

Threatened/Endangered Fish

0.90

Upstream/Downstream Threatened/Endangered Wildlife

0.90

wild/Scenic Location

0.90 Federal Land Code 103
0.90 Federal Land code 104

0.90

Cultural value

0.50

0.90 Federal Land Code 105

0.90

0.90 Federal Land Code 106

0.90

Geologic Value

0.90 Federal Land Code 107

0.90

other Value

0.90 Federal Land Code 108

0.90

Recreation value

0.90

Federal Land Code 198

0.90

Scenic Value

0.90 
DATE: $10 / 31 / 97$

FERC

Number

11139
Plant Name

TERROR LAKE

County Name

KODIAK DIVISION

Class

c

KODIAK ELEC ASSN, INC

Name Plate

Rating $(\mathrm{KW})$

3000.00

TERROR R
Annual Energy

PESF*KW

PAGE NO: 45

State

stream

Name

AK

River Basin

KODIAK ISLAND RIVER BASINS

$\begin{array}{cccllr}\text { Unit } & \text { Plant } & \text { Project } & \text { Dam } & \text { Latitude } & 5740.00 \\ \text { TYpe } & \text { Type } & \text { Status } & \text { Status } & \text { Longitude } 15305.00 \\ \text { C } & \text { ROR } & \text { XX } & \text { Wo } & \end{array}$

Factor

Wild/Scenic Protection

Wild/Scenic Tributary or

Upstream/ Downstream

wild/Scenic Location

Cultural Value

Fish Presence Value

Geologic Value

Historic Value

other value

Recreation value

Scenic Value
Exist Prob

Factor

Exist Prob

0.90

Wildlife Value Threatened/Endangered Fish Threatened/Endangered Wildlife

0.90 Federal Land Code 103

0.90 Federal Land Code 104

0.90 Federal Land Code 105

0.90 Federal Land Code 106

0.90 Federa.l Land Code 107

0.90 Federal Land Code 108

0.90 Federal Land Code 198

0.90
PESF Annual Energy Rating (MWh)

6000 
DATE: $10 / 31 / 97$

PAGE NO: 46

FERC

Number

Plant Name

11243 POWER CREEK

County Name

CORDOVA-MCCARTHY DIV
River Basin

KENAI PENINSULA RIVER BAS

\section{Class Owner Name}

$R$ WHITEWATER ENGINEERING CORP

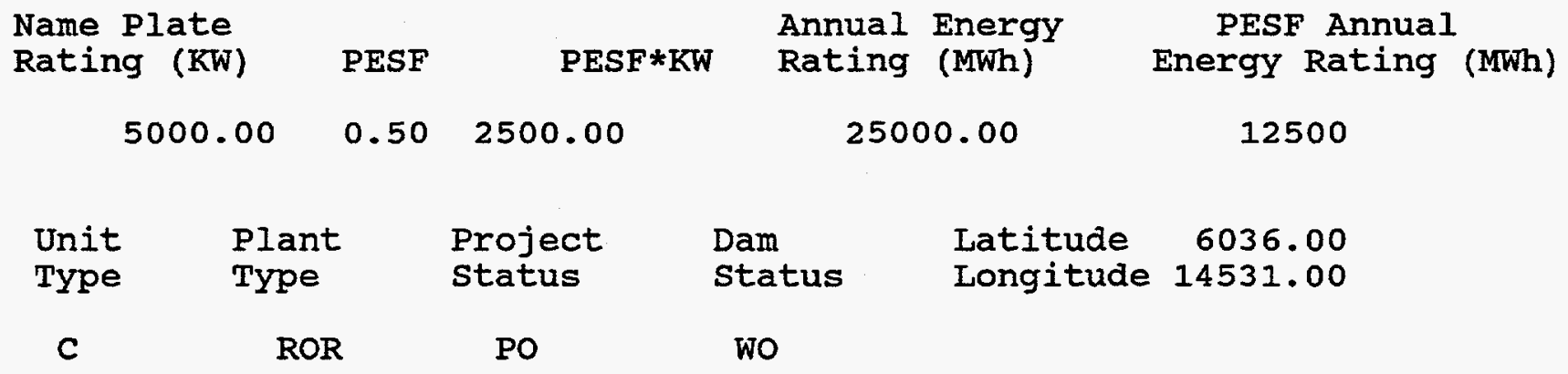

Factor

wild/Scenic Protection Wild/Scenic Tributary or Upstream/Downstream wild/Scenic Location Cultural value Fish Presence Value Geologic Value Historic Value other Value Recreation Value Scenic Value
Exist Prob

Factor

Exist Prob

0.90 Wildlife Value Threatened/Endangered Fish Threatened/Endangered Wildlife

0.90

0.90

$\begin{array}{lll}0.90 & \text { Federal Land Code } 103 \\ 0.90 & \text { Federal Land Code } 104\end{array}$

$\mathrm{Y} \quad 0.75$ Federal Land code 105

0.90 Federal Land code 106

0.90 Federal Land Code 107

0.90 Federal Land Code 108

$\mathrm{Y} \quad 0.75$

Federal Land Code 198

0.90

0.90

$\mathrm{Y} \quad 0.75$

0.90

0.90

0.90

0.90

0.90
State Name

AK

0.90 
DATE: $10 / 31 / 97$

FERC

Number

11244

SILVER LAKE

County Name

VALDEZ

Class

M WHITEWATER ENGINEERING CORP

Name Plate

Rating ( $\mathrm{KW})$

PESF

PESF*KW

Annual Energy

15000.00

$0.25 \quad 3750.00$

Unit

Type

$$
\begin{gathered}
\text { Plant } \\
\text { Type } \\
\text { STG }
\end{gathered}
$$

c

Factor

Exist Prob

Factor

Latitude 6057.00

Project status

PO

\section{status}

Longitude 14633.00
State

Name

AK
PESF Annual

Energy Rating (MWh)

14000
Wild/Scenic Protection

wild/Scenic Tributary or

Upstream/Downstream

wild/Scenic Location

Cultural value

Fish Presence Value

Geologic Value

Historic Value

other value

Recreation Value

Scenic Value

\section{Exist Prob}

0.90

\section{Wildlife Value}

Threatened/Endangered Fish

Threatened/Endangered Wildlife

0.90 Federal Land Code 103

0.90 Federal Land Code 104

Y $\quad 0.25$ Federal Land Code 105

0.90 Federal Land Code 106

0.90 Federal Land Code 107

0.90 Federal Land Code 108

0.90 Federal Land Code 198

0.90
Exist Prob

0.90

0.90

0.90

0.90

0.50

0.90

0.90

0.90

0.90

0.90 
FERC

Number

11287
Plant Name

LACE RIVER

County Name

JUNEAU DIVISION

\section{Class}

$\mathbf{R}$

LACE RIVER HYDRO

Name Plate

Rating ( $\mathrm{KW}$ )

PESF

$\mathrm{PESF} * \mathrm{KW}$

Annual Energy

Rating (MWh)

4900.00

0.75

3675.00
River Basin

JUNEAU GROUP

$$
34164.00
$$

State

Name

AK

$\begin{array}{cccll}\text { Unit } & \text { Plant } & \text { Project } & \text { Dam } & \text { Latitude } \\ \text { Type } & \text { Type } & \text { Status } & \text { Status } & \text { Longitude } 13450.00 \\ & \text { ROR } & \text { Po } & \text { Wo } & \end{array}$

Factor

Exist Prob

0.90

wild/Scenic Protection

Wild/Scenic Tributary or Upstream/Downstream wild/Scenic Location Cultural Value

Fish Presence Value Geologic Value Historic Value other Value Recreation value Scenic Value
Factor

Exist Prob
PESF Annual
nergy Rating (MWh)

25623

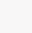


RESOU R C E

DATE: $10 / 31 / 97$
D A T A B A S E

FERC

Number

Plant Name

11290 TAIYA INLET

county Name

SKAGWAY-YAKUTAT DIV

JUNEAU GROUP

River Basin
L I S T I N G

PAGE NO: 49

State

Name

DAYEBAS CR, KATZEHIN $R \quad$ AK

$\begin{array}{cr}\text { Class } & \text { Owner Name } \\ \text { R } & \text { TAIYA INLET HYDRO }\end{array}$

Name Plate Rating ( $\mathrm{KW}$ ) PESF

Annual Energy

$$
2000.00 \quad 0.75 \quad 1500.00
$$

$\mathrm{PESF} * \mathrm{KW}$

Rating (MWh)

PESF Annual

$\begin{array}{cc}2000.00 & 0.7 \\ \text { Unit } & \begin{array}{c}\text { Plant } \\ \text { Type }\end{array} \\ \text { C } & \text { ROR } \\ \text { Factor } & \end{array}$

Wild/Scenic Protection

wild/Scenic Tributary or

Upstream/Downstream

wild/Scenic Location

Exist Prob

Factor

Exist Prob

$\begin{array}{llr}\text { Dam } & \text { Latitude } & 5917.00 \\ \text { Status } & \text { Longitude } & 13502.00\end{array}$

wo Energy Rating (MWh)

10402.50

Cultural Value

Fish Presence Value

Project
Status

13870.00

Geologic Value

Historic Value

other value

Recreation value

Scenic Value
0.90 Wildife Value
Threatened/Endangered Fish
Threatened/Endangered Wildlife
0.90 Federal Land Code 103
0.90 Federal Land Code 104
0.90 Federal Land Code 105
0.90 Federal Land Code 106
0.90 Federal Land Code 107
0.90 Federal Land Code 108
0.90 Federal Iand Code 198

0.90

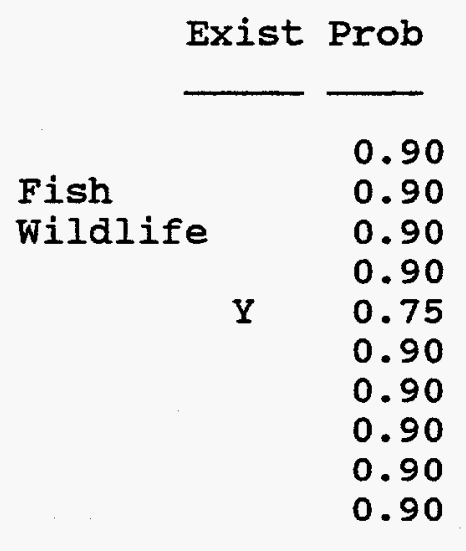


FERC

Number

11316
Plant Name TAZIMINA RIVER

County Name

BRISTOL BAY BORO DIV
State

Name

AK

\section{Class Owner Name \\ C ILIAMNA-NEWHALEM-NONDALTON ELEC}

Name Plate Rating ( $\mathrm{KW})$

700.00
PESF

Annual Energy PESF*KW Rating (MWh)
PESF Annual Energy Rating (MWh)
3000.00
$0.10 \quad 70.00$
300

$$
\begin{gathered}
\text { Project } \\
\text { Status } \\
\text { Po }
\end{gathered}
$$

Exist Prob

Factor

Latitude 5957.00

$\begin{array}{llr}\text { Dam } & \text { Latitude } & 5957.00 \\ \text { Status } & \text { Longitude } & 15433.00\end{array}$

U
Factor

wild/Scenic Protection wild/Scenic Tributary or Upstream/Downstream wild/Scenic Location Cultural value Fish Presence Value Geologic Value Historic Value other Value Recreation Value Scenic Value

\section{Exist Prob}

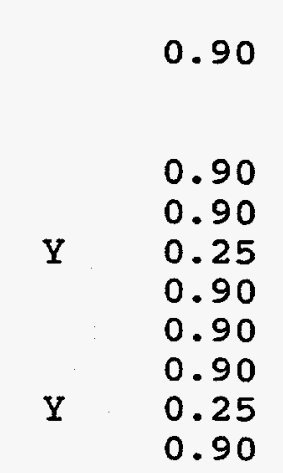

Wildlife Value Threatened/Endangered Fish Threatened/Endangered Wildlife Exist Prob Federal Land Code 103 Federal Land Code 104 Federal Land Code 105 Federal Land Code 106 Federal Land Code 107 Federal Land Code 108 Federal Land Code 198

0.90

0.90

0.90

0.90

0.90

0.90

0.90

0.90

0.90

0.90 
DATE: $10 / 31 / 97$

FERC

Number

11319

HAINES
Plant Name

County Name

FIRST JUDICIAL DISTRICT
PAGE NO: 51

State

Stream

Name

AK
Class
Owner Name
$R$ HAINES IIGHT AND POWER CO, INC

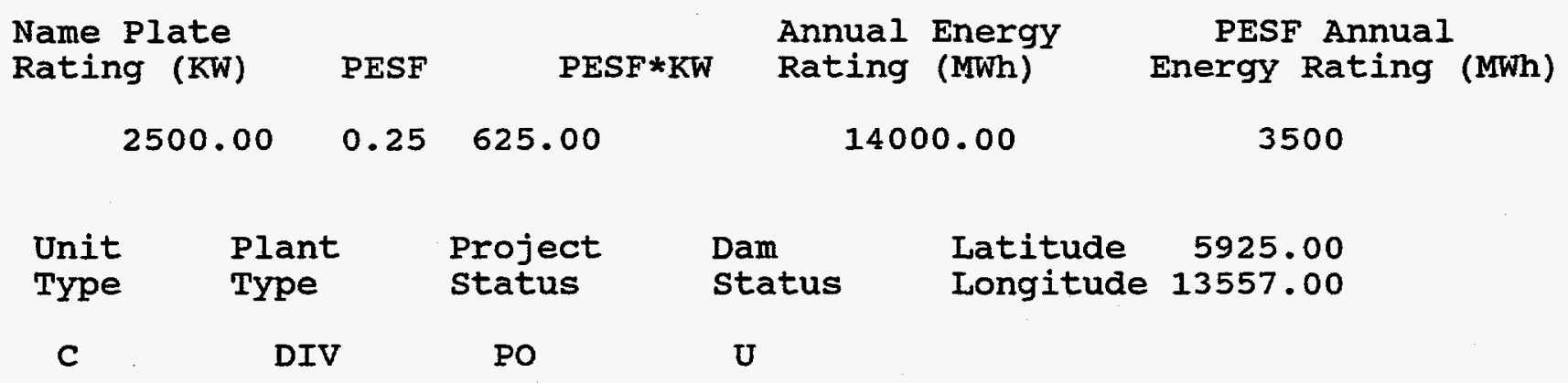

Factor

Exist Prob

0.90

Wildlife Value Threatened/Endangered Fish Threatened/Endangered Wildlife

0.90 Federal Land Code 103

0.90 Federal Land Code 104

0.90 Federal Land Code 105

0.90 Federal Land code 106

0.90 Federal Land Code 107

0.90 Federal Land Code 108

0.90

0.90
Exist Prob

$\mathrm{Y}$

0.25

0.90

0.90

0.90

0.50

0.90

0.90

0.90

0.90

0.90 
DATE: $10 / 31 / 97$

PAGE NO: 52

FERC

Number

11393
Plant Name

MOHONEY LAKE

County Name

KETCHIKAN DIVISION
Stream

MAHONEY CR

River Basin

KETCHIKAN GROUP state

Name

AK $\begin{array}{cc}\text { Class } & \text { Owner Name } \\ \text { R } & \text { CITY OF SAXMAN }\end{array}$

Name Plate Rating ( $\mathrm{KW}$ )

\section{PESF}

0.252250 .00
Annual Energy

Rating (MWh)

32450.00
PESF Annual Energy Rating (MWh)

\begin{tabular}{|c|c|c|c|c|}
\hline $\begin{array}{l}\text { Unit } \\
\text { Type }\end{array}$ & $\begin{array}{l}\text { Plant } \\
\text { Type }\end{array}$ & $\begin{array}{l}\text { Project } \\
\text { status }\end{array}$ & $\begin{array}{l}\text { Dam } \\
\text { status }\end{array}$ & $\begin{array}{lr}\text { Latitude } & 5527.00 \\
\text { Longitude } & 13131.00\end{array}$ \\
\hline C & ROR & PO & $\mathrm{U}$ & \\
\hline
\end{tabular}

Factor

Exist Prob

Factor

Exist Prob

Wild/Scenic Protection

wild/Scenic Tributary or

Upstream/Downstream

wild/Scenic Location

Cultural Value

Fish Presence Value

Geologic Value

Historic Value

other value

Recreation value

Scenic Value

Exist Prob

0.90

Wildlife Value

Threatened/Endangered Fish

Threatened/Endangered Wildlife

0.90 Federal Land code 103

0.90 Federal Land Code 104

Y $\quad 0.25$ Federal Land Code 105

0.90 Federal Land Code 106

0.90 Federal Land Code 107

0.90 Federal Land Code 108

0.90 Federal Land Code 198
8112.50 
DATE: $10 / 31 / 97$

FERC

Number

Plant Name

11417 SNYDER FALLS CREEK

County Name

CORDOVA-MCCARTHY DIV
PAGE NO: 53

State

Name

AK

SNYDER FALLS CR, NELSON BAY

River Basin

KENAI PENINSULA RIVER BAS

class Owner Name

R DAVID AUSMAN \& ASSOC

Name Plate

Rating (KW)

PESF

Annual Energy

PESF*KW

Rating (MWh)

PESF Annual

1450.00

$0.75 \quad 1087.50$

8000.00

Energy Rating (MWh)

$0.75 \quad 1087.50$

$\begin{array}{lll}\text { Unit } & \text { Plant } & \text { Project } \\ \text { Type } & \text { Type } & \text { Status }\end{array}$

Type

C

ROR

PO

Dam

Status

Latitude 6050.00

พั

Factor

Wild/Scenic Protection

Wild/Scenic Tributary or

Upstream/Downstream

wild/Scenic Location

cultural value

Fish Presence Value

Geologic Value

Historic Value

other Value

Recreation Value

Scenic Value
Exist Prob

0.90

Wildlife Value

Threatened/Endangered Fish

Threatened/Endangered wildlife

0.90 Federal Land Code 103

0.90 Federal Land Code 104

0.90 Federal Land Code 105

0.90 Federal Land Code 106

0.90 Federal Land Code 107

0.90 Federal Land Code 108

0.90 Federal Land code 198
Exist Prob

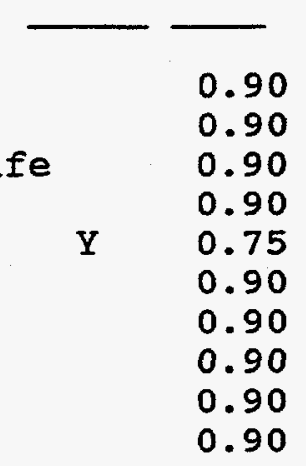


RE SOU R C E D A T A B A S E L I S T I N G

DATE: $10 / 31 / 97$

PAGE NO: 54

FERC

Number

Plant Name

stream

State

Name

11480 UPPER REYNOLDS CREEK

REYNOLDS CR, COPPER HARBOR

AK

County Name

River Basin

PRINCE OF WALES DIV

KETCHIKAN GROUP

Class Owner Name

C HAIDA CORPORATION

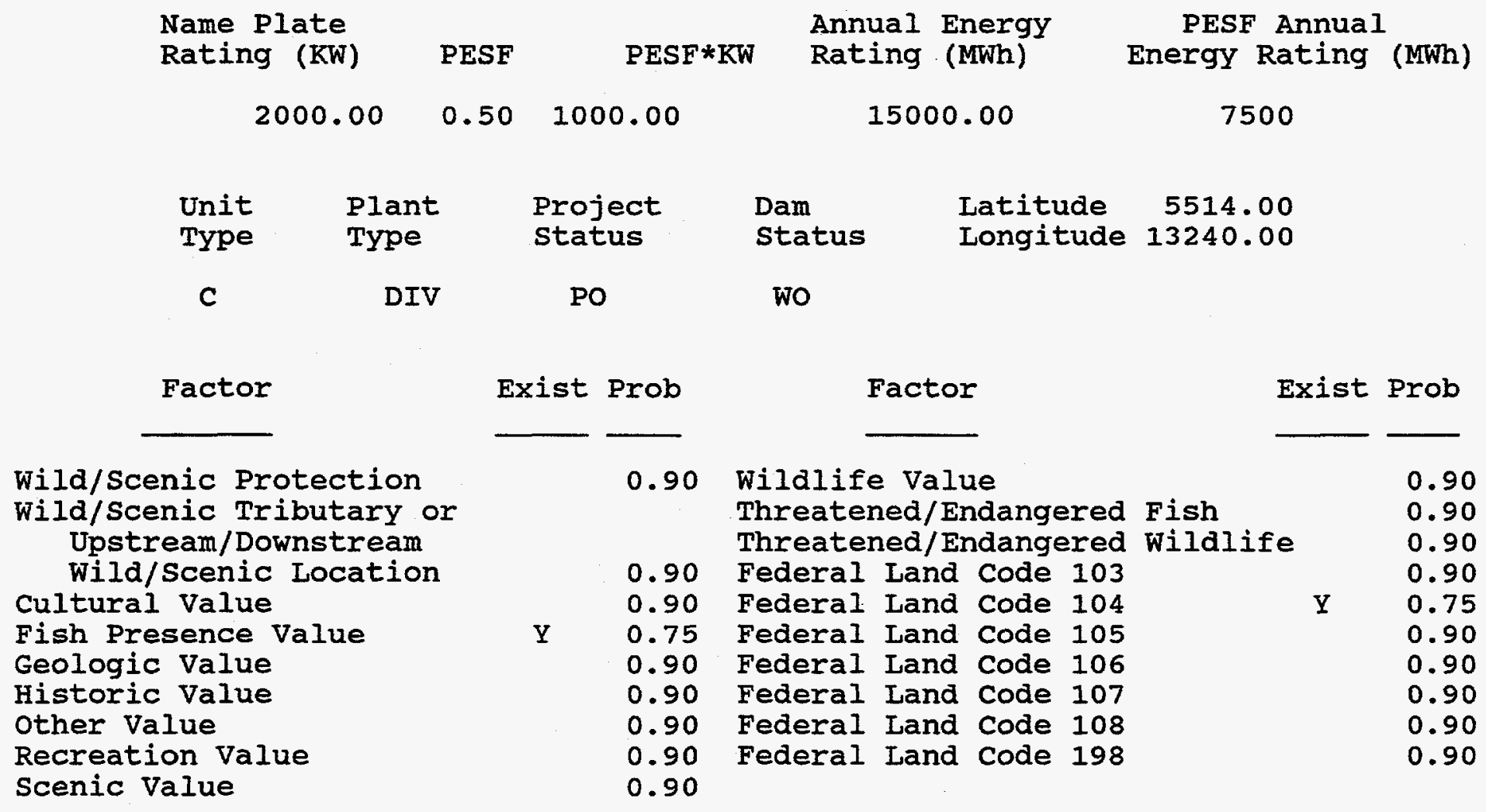


DATE: $10 / 31 / 97$

FERC

Number

11497
Plant Name

LEANNE LAKE

County Name

KODIAK DIVISION
PAGE NO: 55

State

stream

Name

LEANNE LAKE OUTLET

AK

$$
\begin{array}{cc}
\text { Class } & \text { Owner Name } \\
\text { C } & \text { KODIAK ELECTRIC ASSOCIATION, INC }
\end{array}
$$

Name Plate Rating (KW)

$$
2800.00
$$

PESF

PESF*KW

Annual Energy Rating (MWh)

$$
80000.00
$$

2520.00

Unit

Plant

Type

Type

C

ROR

Factor

Exist Prob

Factor

Exist Prob

\subsection{Wildlife Value}

Threatened/Endangered Fish

Wild/Scenic Tributary or

Upstream/Downstream

wild/Scenic Location

Cultural Value

Fish Presence Value

Geologic Value

Historic Value

other Value

Recreation Value

Scenic Value
Threatened/Endangered Wildlife

0.90 Federal Land Code 103

0.90 Federal Land Code 104

0.90 Federal Land Code 105

0.90 Federal Land Code 106

0.90 Federal Land Code 107

0.90 Federal Land Code 108

0.90 Federal Land Code 198
PESF Annual Energy Rating (MWh)

72000

5744.00 
DATE: $10 / 31 / 97$

PAGE NO: 56

FERC

Number

Plant Name

stream

State

11508

WOLF LAKE

WOLF CR

Name

11508

County Name

River Basin

PRINCE OF WALES DIV

KETCHIKAN GROUP

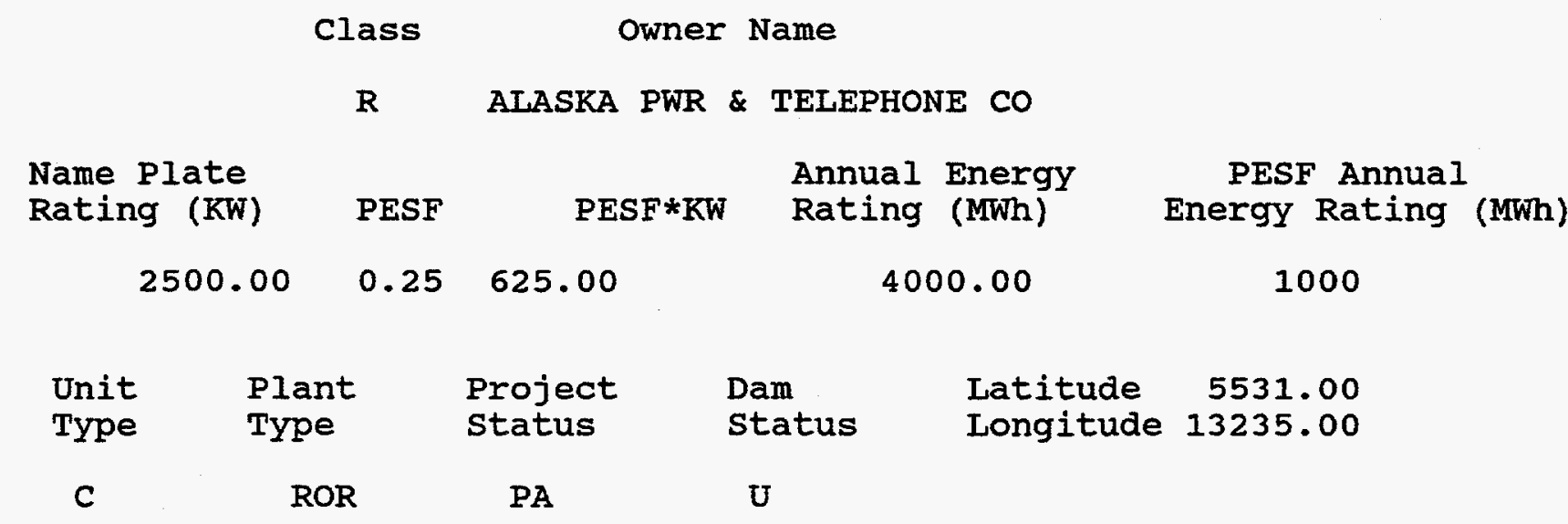

Factor

Exist Prob

Factor

Exist Prob

Wild/Scenic Protection

wild/Scenic Tributary or

Upstream/Downstream

wild/Scenic Location

Cultural Value

Fish Presence Value

Geologic Value

Historic Value

other value

Recreation Value

Scenic Value

\begin{tabular}{|c|c|c|c|c|c|}
\hline \multirow{8}{*}{$\mathrm{Y}$} & 0.90 & $\begin{array}{l}\text { Wildlife } \\
\text { Threaten } \\
\text { Threaten }\end{array}$ & $\begin{array}{l}\text { Val } \\
\text { led/F } \\
\text { led/E }\end{array}$ & $\begin{array}{l}\text { ue } \\
\text { ndangered } \\
\text { ndangered }\end{array}$ & \multirow[t]{8}{*}{$\begin{array}{l}\text { Fish } \\
\text { wildlife }\end{array}$} \\
\hline & 0.90 & Federal & Land & Code 103 & \\
\hline & 0.90 & Federal & Land & Code 104 & \\
\hline & 0.25 & Federal & Land & Code 105 & \\
\hline & 0.90 & Federal & Land & Code 106 & \\
\hline & 0.90 & Federal & Land & Code 107 & \\
\hline & 0.90 & Federal & Land & Code 108 & \\
\hline & 0.90 & Federal & Land & Code 198 & \\
\hline
\end{tabular}

0.90

0.90

0.90

0.90

$\mathrm{Y}$

0.50

0.90

0.90

0.90

0.90

0.90 
DATE: $10 / 31 / 97$

PAGE NO: 57

FERC

Number

Plant Name

stream

State

11510 ALIISON

ALLISON CR

Name

AK

county Name

VALDEZ-CHITINA-WHITT DV
River Basin

KENAI PENINSULA RIVER BAS

\section{Class Owner Name}

F ALASKA BUSINESS; INDUSTRIAL DEV.CORP

Name Plate

Rating (KW)

8000.00
PESF

0.252000 .00
Annual Energy Rating (MWh)

4350.00
PESF Annual Energy Rating (MWh)

$\begin{array}{cccc}\text { Unit } & \begin{array}{l}\text { Plant } \\ \text { Type }\end{array} & \begin{array}{l}\text { Project } \\ \text { Status }\end{array} & \begin{array}{l}\text { Dam } \\ \text { Status }\end{array} \\ \text { C } & \text { STG } & \text { PA } & \text { U }\end{array}$

Factor

Wild/Scenic Protection

Wild/Scenic Tributary or Upstream/Downstream wild/Scenic Location Cultural value Fish Presence Value Geologic Value Historic Value other value Recreation value Scenic Value
Exist Prob

0.90

Wildlife Value Threatened/Endangered Fish Threatened/Endangered Wildlife

0.90 Federal Land Code 103

0.90 Federal Land Code 104

Y $\quad 0.25$ Federal Land Code 105

0.90 Federal Land Code 106

0.90 Federal Land Code 107

0.90 Federal Land Code 108

0.90 Federal Land Code 198
1087.50

0.90

Exist Prob

0.90

0.90

0.90

0.90

0.90

0.90

0.90

0.90

0.90

0.90 
DATE: $10 / 31 / 97$

PAGE NO: 58

FERC

Number

Plant Name

stream

state

Name

AK001 BROWNE

NENANA R

AR

County Name

River Basin

YUKON-KOYUKUK DIVISION

YUKON RIVER BASIN

Class Owner Name

NHPS / COE

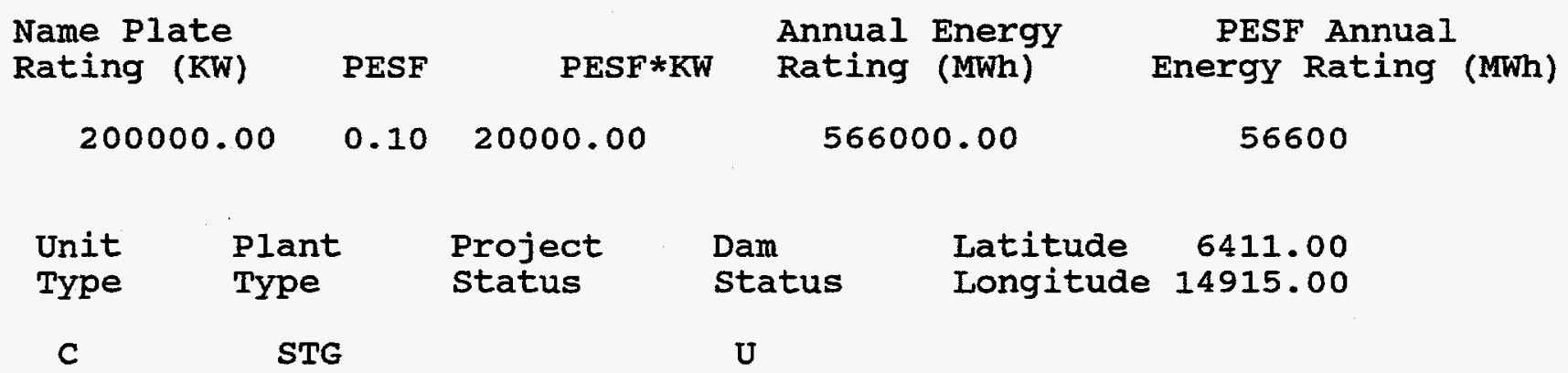

Factor

Exist Prob

Factor

Exist Prob

wild/Scenic Protection

wild/Scenic Tributary or

0.90 Wildife Value

0.90

Threatened/Endangered Fish

0.90

Upstream/Downstream Threatened/Endangered wildlife

0.90

wild/Scenic Location

0.90 Federal Land Code 103

0.90

Cultural value

0.90 Federal Land Code 104

0.90

Fish Presence Value

$\mathrm{Y}$

0.25 Federal Land Code 105

0.90

Geologic Value

0.90 Federal Land Code 106

0.90

Historic Value

0.90 Federal Land Code 107

0.90

other Value

0.90 Federal Land Code 108

0.90

Recreation Value

Scenic Value

$\mathrm{Y} \quad 0.25$

Federal Land Code 198

0.90 
R E S O U R C E

DATE: $10 / 31 / 97$

FERC

Number

Plant Name

AKOO2 SCAMMON

county Name

WADE HAMPTON DIVISION
D A T A B A S E

I I S T I N G

PAGE NO: 59
State

Name

UNN STR, SCAMMON BAY
AK

$$
\begin{array}{cc}
\text { Class } & \text { Owner Name } \\
\text { C } & \text { AK VILLAGE ELEC COOP, INC }
\end{array}
$$

\begin{tabular}{|c|c|c|c|c|c|c|c|}
\hline $\begin{array}{l}\text { Name } \\
\text { Rating }\end{array}$ & $\begin{array}{l}\text { Plate } \\
g(\mathrm{KW})\end{array}$ & PESF & PESF*KW & $\begin{array}{l}\text { Annt } \\
\text { Rat: }\end{array}$ & $\begin{array}{l}\text { ual } \\
\text { ing }\end{array}$ & $\begin{array}{l}\text { Energy } \\
\text { (MWh) }\end{array}$ & $\begin{array}{l}\text { PESF An } \\
\text { Energy Rat }\end{array}$ \\
\hline & 175.00 & 0.90 & 157.50 & & 138 & 30.00 & 1242 \\
\hline $\begin{array}{l}\text { Unit } \\
\text { Type }\end{array}$ & $\begin{array}{l}\text { Pl: } \\
\text { TYl }\end{array}$ & & $\begin{array}{l}\text { Project } \\
\text { status }\end{array}$ & $\begin{array}{l}\text { Dam } \\
\text { status }\end{array}$ & & $\begin{array}{l}\text { Latitude } \\
\text { Longitude }\end{array}$ & $\begin{array}{r}6148.00 \\
16529.00\end{array}$ \\
\hline c & & & & wo & & & \\
\hline
\end{tabular}

Factor

Exist Prob

Wild/Scenic Protection

wild/Scenic Tributary or

Upstream/Downstream wild/Scenic Location Cultural Value

Fish Presence Value

Geologic Value

Historic Value

other value

Recreation value

Scenic Value
River Basin

YUKON RIVER BASIN 
FERC

Number

Plant Name

AKO03 SCAMMON (PHASE 2)

County Name

WADE HAMPTON DIVISION

\section{stream}

UNN STR, SCAMMON BAY

River Basin

YUKON RIVER BASIN
State

Name

AK

$$
\begin{array}{cc}
\text { Class } & \text { Owner Name } \\
\text { C } & \text { AK VILLAGE ELEC COOP, INC }
\end{array}
$$

Name Plate Rating ( $\mathrm{KW}$ )

125.00

\section{PESF}

$0.90 \quad 112.50$
Annual Energy PESF*KW Rating (MWh)

985.00
PESF Annual Energy Rating (MWh)

886.50

$\begin{array}{cclll}\text { Unit } & \text { Plant } & \text { Project } & \text { Dam } & \text { Latitude } \\ \text { Type } & \text { Type } & \text { Status } & \text { Status } & \text { Longitude } 16529.00 \\ \text { C } & \text { DIV } & & \text { wo } & \end{array}$

Factor

Exist Prob

Factor

Exist Prob

Wild/Scenic Protection

wild/Scenic Tributary or

Upstream/Downstream

wild/Scenic Location

Cultural Value

Fish Presence Value

0.90 Wildlife Value

Threatened/Endangered $\mathrm{Fish}$

Threatened/Endangered Wildlife

0.90

$\begin{array}{ll}0.90 & \text { Federal Land Code } 103 \\ 0.90 & \text { Federal Land Code } 104\end{array}$

0.90

$\begin{array}{lll}0.90 & \text { Federal Land Code } 104 \\ 0.90 & \text { Federal Land Code } 105\end{array}$

0.90

0.90 Federal Land Code 106

0.90

0.90 Federal Land Code 106

0.90

Geologic Value

Historic Value

other Value

0.90 Federal Land Code 108

0.90

0.90 Federal Land Code 198

0.90

0.90

Recreation value

0.90

0.90 
DATE: $10 / 31 / 97$

FERC

Number

Plant Name

AKO04

LAKE ELVA

County Name

BRISTOL BAY BORO DIV
PAGE NO: 61

State

Name

AK

\section{Class Owner Name}

M ALASKA ENERGY AUTH

Name Plate Rating (KW)

1500.00
PESF

$0.75 \quad 1125.00$
Annual Energy Rating (MWh)

6000.00
PESF Annual Energy Rating (MWh)

4500

\begin{tabular}{|c|c|c|c|c|}
\hline $\begin{array}{l}\text { Unit } \\
\text { Type }\end{array}$ & $\begin{array}{l}\text { Plant } \\
\text { Type }\end{array}$ & $\begin{array}{l}\text { Project } \\
\text { status }\end{array}$ & $\begin{array}{l}\text { Dam } \\
\text { status }\end{array}$ & $\begin{array}{lr}\text { Latitude } 5938.00 \\
\text { Longitude } 15900.00\end{array}$ \\
\hline C & STG & & wo & \\
\hline
\end{tabular}

Factor

Exist Prob

Factor

wild/Scenic Protection

wild/Scenic Tributary or

Upstream/Downstream

wild/Scenic Location

Cultural Value

Fish Presence Value

Geologic Value

Historic Value

other value

Recreation Value

Scenic Value

0.90 Wildlife Value Threatened/Endangered Fish Threatened/Endangered Wildlife

0.90 Federal Land Code 103

0.90 Federal Land Code 104

Y

0.75

0.90

Federal Land Code 105

Federal Land Code 106

Federal Land code 107

0.90 Federal Land Code 108

0.90 Federal Land Code 198
Exist Prob

\section{Exist Prob}

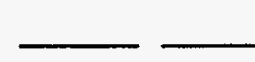

0.90

0.90

0.90

0.90

0.90

0.90

0.90

0.90

0.90

0.90 
FERC

Number

AKO05
Plant Name

GRANT LAKE

County Name

BRISTOL BAY BORO DIV

\section{stream}

WOOD $R$, KOKWOK $R$

River Basin

KOTZEBUE-NUSHAGAK RIVER B state

Name

AK

\section{Class Owner Name}

NHPS/COE

Name Plate Rating (KW)

2700.00

\section{PESF}

0.25

675.00
Annual Energy Rating (MWh)

12700.00
PESF Annual Energy Rating (MWh)

$\begin{array}{cclll}\text { Unit } & \text { Plant } & \text { Project } & \text { Dam } & \text { Latitude } \\ \text { Type } & \text { Type } & \text { Status } & \text { Status } & \text { Longitude } 15832.00 \\ \text { C } & \text { STG } & & \text { U } & \end{array}$

Factor

Exist Prob

Factor

Exist Prob

wild/scenic Protection

Wild/Scenic Tributary or

Upstream/Downstream

wild/Scenic Location

Cultural value

Fish Presence Value

Geologic Value

Historic Value

other Value

Recreation value

Scenic Value

Exist Prob

0.90

Wildlife value

Threatened/Endangered Fish

Threatened/Endangered Wildlife

0.90 Federal Land Code 103

0.90 Federal Land Code 104

Y $\quad 0.25$ Federal Land Code 105

0.90 Federal Land Code 106

0.90 Federal Land Code 107

0.90 Federal Land Code 108

0.90 Federal Land Code 198
0.90

0.90

0.90

0.90

0.90

0.90

0.90

0.90

0.90

0.90 
DATE: $10 / 31 / 97$

FERC

Number

Plant Name

AK006 NUYAKUK (TIKCHIK)

County Name

BRISTOL BAY BORO DIV

Class

Owner Name

Name Plate

Rating ( $\mathrm{KW}$ )

PESF

PESF*KW

Annual Energy

Rating (MWh)

PAGE NO: 63

NUYAKUK $\mathbf{R}$

stream

State

Name

AK
River Basin

KOTZEBUE-NUSHAGAK RIVER B

$$
127000.00
$$

0.7595250 .00

Unit

Type

$$
\text { Plant }
$$
Type

\section{Project} status

c STG
PESF Annual Energy Rating (MWh)
Factor

wild/Scenic Protection Wild/Scenic Tributary or Upstream/Downstream wild/Scenic Location Cultural Value Fish Presence Value Geologic Value Historic Value other Value Recreation value Scenic Value
Exist Prob

0.90 Wildlife Value Threatened/Endangered Fish Threatened/Endangered Wildlife

0.90 Federal Land code 103

0.90 Federal Land Code 104

$\mathbf{Y}$

0.75

0.90

Federal Land Code 105

0.90

Federal Land Code 106

0.90

Federal Land Code 107

0.90

0.90

$$
555000.00
$$

Latitude Langitude 5955.00

416250
Dam พo
Factor

Exist Prob

0.90

0.90

0.90

0.90

0.90

0.90

0.90

0.90

0.90

0.90 
R E S O U R C E D A T A B A S E I I S T I N G

DATE: $10 / 31 / 97$

PAGE NO: 64

FERC

Number

Plant Name

Stream

state

Name

AK007 AKUTAN

UNN STR

AK

County Name

River Basin

ALEUTIAN ISLANDS DIV

ALASKA PENINSULA RIVER BAS

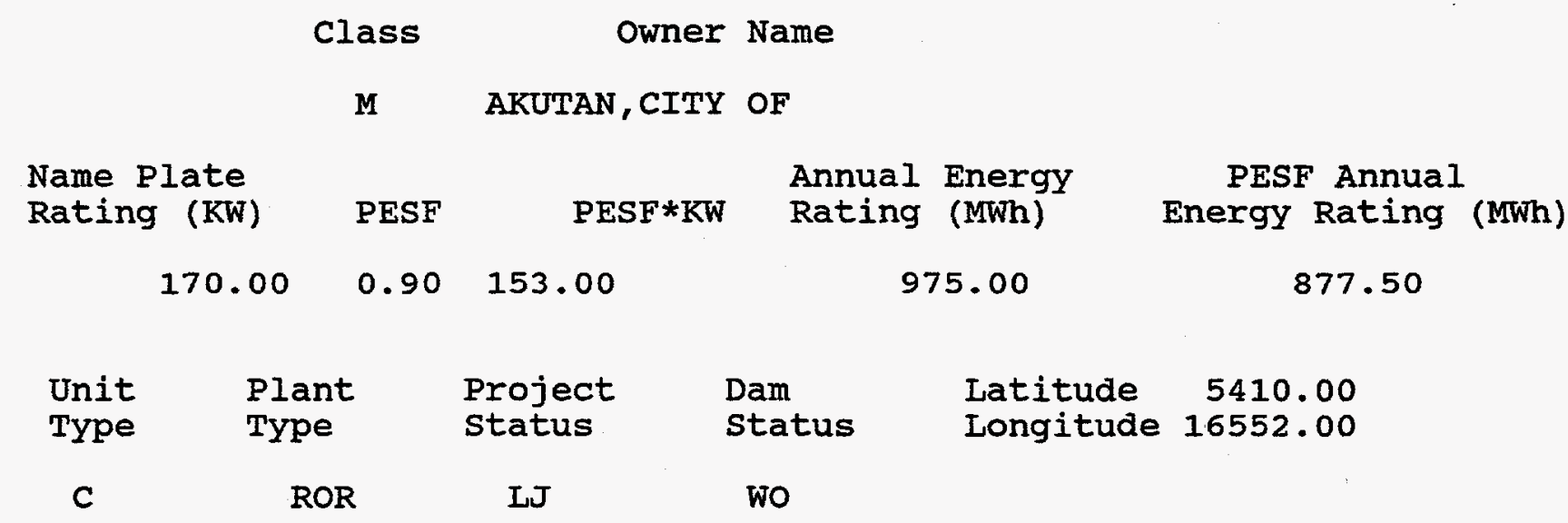

Factor

Wild/Scenic Protection

wild/Scenic Tributary or Upstream/Downstream wild/Scenic Location Cultural value

Fish Presence Value

Geologic Value

Historic Value other Value

Recreation Value

Scenic Value
Exist Prob

0.90

Wildlife Value

Threatened/Endangered Fish

Threatened/Endangered Wildlife

0.90 Federal Land Code 103

0.90 Federal Land Code 104

0.90 Federal Land code 105

0.90 Federal Land Code 106

0.90 Federal Land Code 107

0.90 Federal Land Code 108

0.90 Federal Land Code 198

Exist Prob

0.90

0.90

0.90

0.90

0.90

0.90

0.90

0.90

0.90

0.90

0.90 
R E S O U R C E

DATE: $10 / 31 / 97$

FERC

Number

Plant Name

AKO08 INDIAN CREEK

County Name

ALEUTIAN ISLANDS DIV
D A T A B A S E

\author{
D A T A B A $\mathbf{S}$ E
}

PAGE NO:
INDIAN CR, CHIGNIK BAY
River Basin
ALASKA PENINSULA RIVER BAS
OWnET Name
OF ENGINEERS
Class Owner Name
F CORPS OF ENGINEERS

State

Name

AK

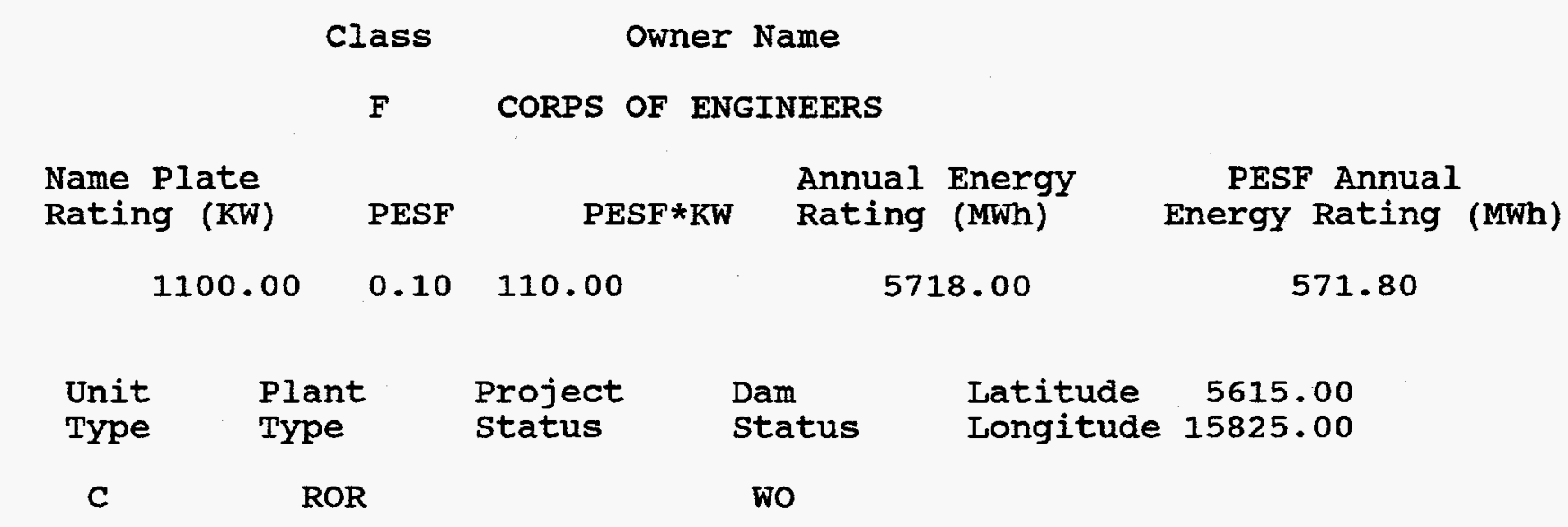

Factor

Wild/Scenic Protection

Wild/Scenic Tributary or

Upstream/ Downstream

wild/Scenic Location

Cultural value

Fish Presence Value

Geologic Value

Historic Value

other Value

Recreation value

Scenic Value
Exist Prob

0.90

Wildife Value

Threatened/Endangered Fish

Threatened/Endangered Wildlife

0.90 Federal Land Code 103

0.90 Federal Land Code 104

0.90 Federal Land Code 105

0.90 Federal Land Code 106

0.90 Federal Land Code 107

0.90 Federal Land Code 108

0.90 Federal Land Code 198

0.90
Exist Prob

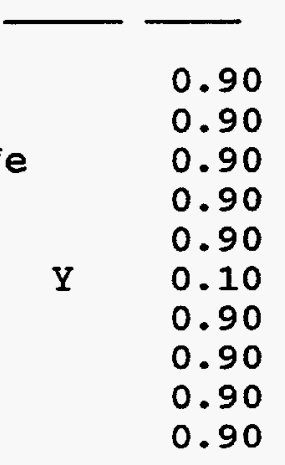


FERC

Number

AR009 ILIAMNA

County Name

BRISTOL BAY BORO DIV
Plant Name

KVICHAK R
State

Name

AR

\section{Class Owner Name}

NHPS / COE

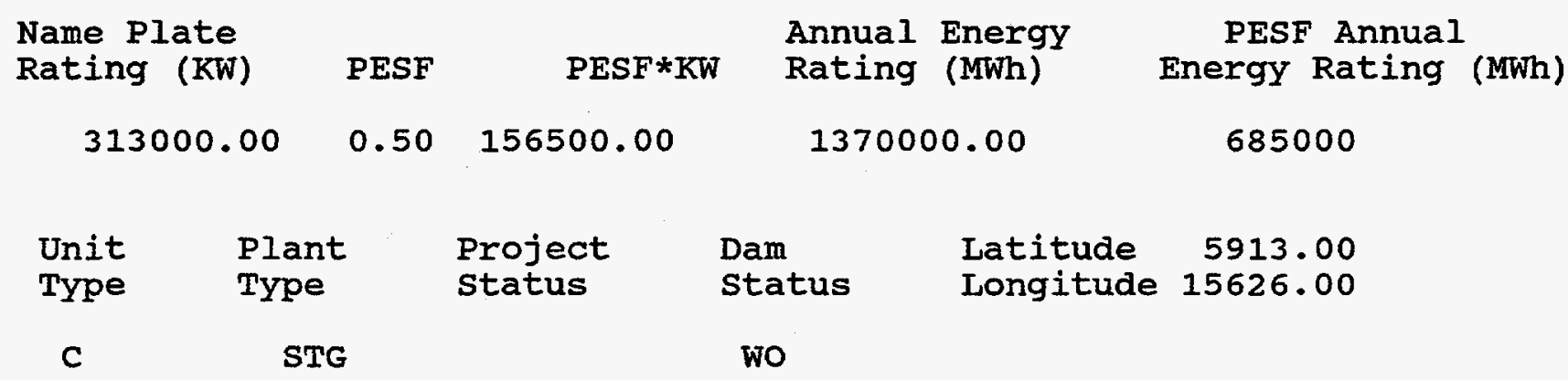

Factor

Wild/Scenic Protection

wild/Scenic Tributary or Upstream/ Downstream

wild/Scenic Location Cultural value Fish Presence Value Geologic Value Historic Value other Value Recreation Value Scenic Value
Exist Prob

0.90

Wildlife Value Threatened/Endangered Fish Threatened/Endangered Wildlife

Y $\quad 0.75$ Federal Land Code 103

0.90 Federal Land Code 104

Y $\quad 0.75$ Federal Land Code 105

0.90 Federal Land Code 106

0.90 Federal Land Code 107

0.90 Federal Land Code 108

Y $\quad 0.75$ Federal Land Code 198
Exist Prob

0.90

0.90

0.90

0.90

0.90

0.90

0.90

0.90

0.90

0.90 
DATE: $10 / 31 / 97$

FERC

Number

Plant Name

AK010

TAZIMINA

County Name

BRISTOL BAY BORO DIV
PAGE NO: 67

State

Name

AK

\section{Class Owner Name}

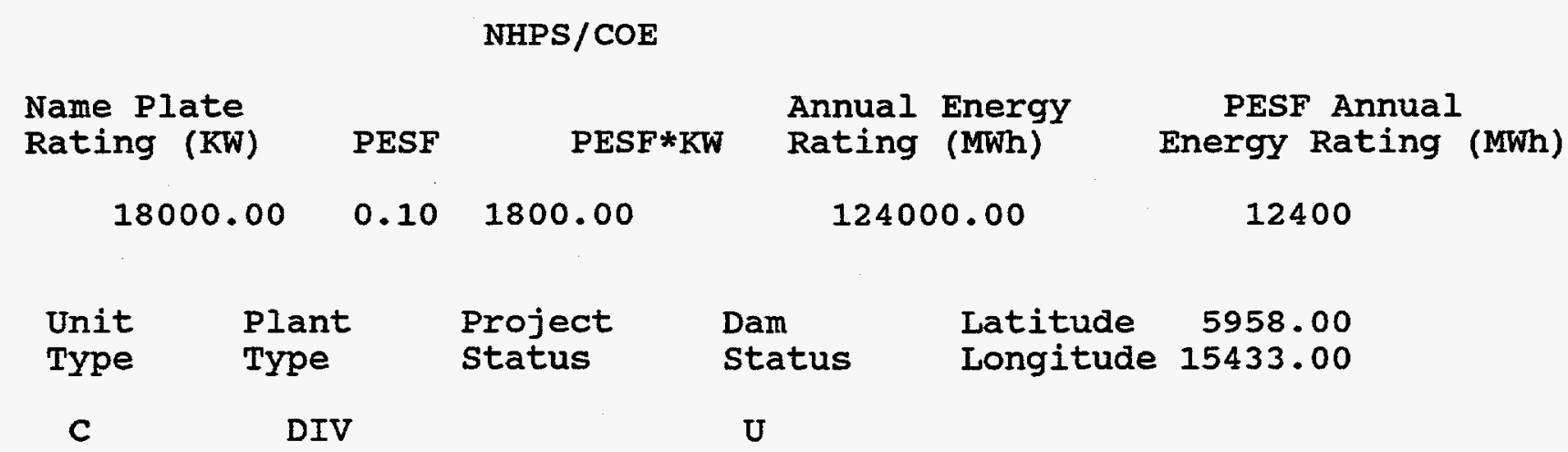

Factor

Exist Prob

\subsection{0}

Wild/Scenic Protection

wild/Scenic Tributary or

Upstream/ Downstream

wild/Scenic Location Cultural value

Fish Presence Value

Geologic Value

Historic Value

other Value

Recreation value

Scenic Value
TAZIMINA $\mathrm{R}$

River Basin

ALASKA PENINSULA RIVER BAS
Stream 
DATE : $10 / 31 / 97$

PAGE NO: 68

FERC

Number

Plant Name

Stream

State

AK011 PORT LIONS

MENNONITE CR, SETTLERS COVE

Name

AK

county Name

KODIAK DIVISION

Class

C KODIAK ELEC ASSN, INC

Name Plate

Rating (KW)

PESF

PESF*KW

Annual Energy

Rating (MWh)

875.00

$0.25 \quad 50.00$

200.00

Plant

Type

Project

status

LJ

STG

Factor

Exist Prob

Factor

Exist Prob

wild/Scenic Protection

wild/Scenic Tributary or Upstream/Downstream wild/Scenic Location

Cultural value

Fish Presence Value

Geologic Value

Historic Value

other Value

Recreation value

Scenic Value
Dam
status
Latitude
5753.00
Longitude 15257.00

PESF Annual

218.75
(MWh)

U

$\begin{array}{lll}0.90 & \text { Wildlife Value } & 0.90 \\ & \text { Threatened/Endangered Fish } & 0.90 \\ & \text { Threatened/Endangered Wildlife } & 0.90 \\ 0.90 & \text { Federal Land Code 103 } & 0.90 \\ 0.90 & \text { Federal Land Code 104 } & 0.90 \\ 0.25 & \text { Federal Land Code } 105 & 0.90 \\ 0.90 & \text { Federal Land Code 106 } & 0.90 \\ 0.90 & \text { Federal Land Code 107 } & 0.90 \\ 0.90 & \text { Federal Land Code 108 } & 0.90 \\ 0.90 & \text { Federal Land Code 198 } & 0.90 \\ 0.90 & & \end{array}$


DATE: $10 / 31 / 97$

FERC

Number

AK012
Plant Name

CHAKACHAMNA

County Name

KENAI-COOK INLET DIV
PAGE NO: 69

State

Name

AK

Class Owner Name

NHPS/COE

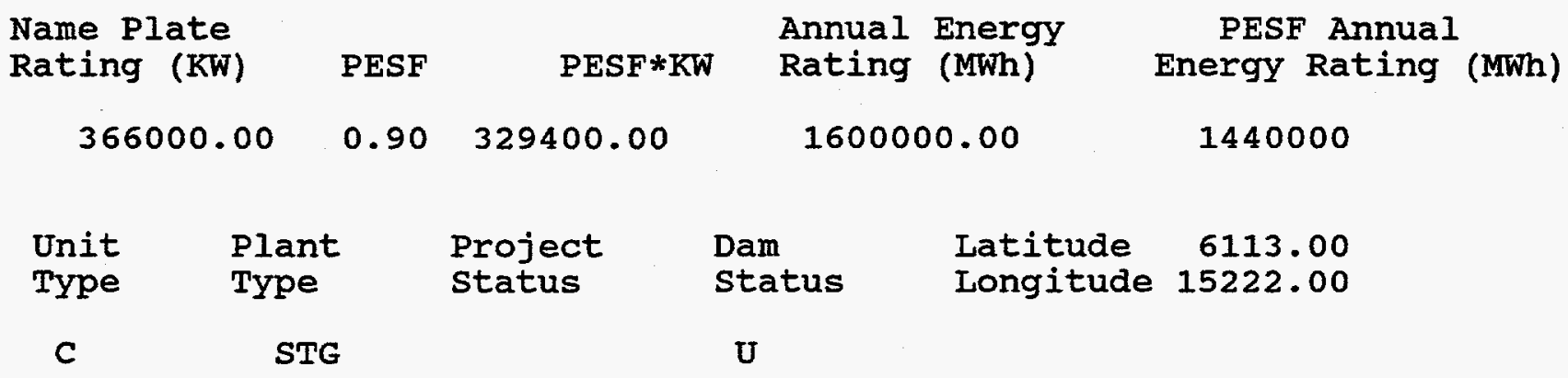

Factor

wild/Scenic Protection

wild/Scenic Tributary or

Upstream/ Downstream

wild/Scenic Location

Cultural value

Fish Presence Value

Geologic Value

Historic Value

other Value

Recreation Value

Scenic Value
Exist Prob

0.90

Wildlife Value

Threatened/Endangered Fish

Threatened/Endangered Wildlife

0.90 Federal Land Code 103

0.90 Federal Land Code 104

0.90 Federal Land Code 105

0.90 Federal Land Code 106

0.90 Federal Land Code 107

0.90 Federal Land Code 108

0.90 Federal Land Code 198

0.90
Exist Prob

0.90

0.90

0.90

0.90

0.90

0.90

0.90

0.90

0.90

0.90 
DATE: $10 / 31 / 97$

FERC

Number

AK013 COFFEE

Plant Name

County Name

RENAI-COOK INLET DIV
PAGE NO: 70

State

Name

BELUGA $R$

AK

Class Owner Name

NHPS/COE

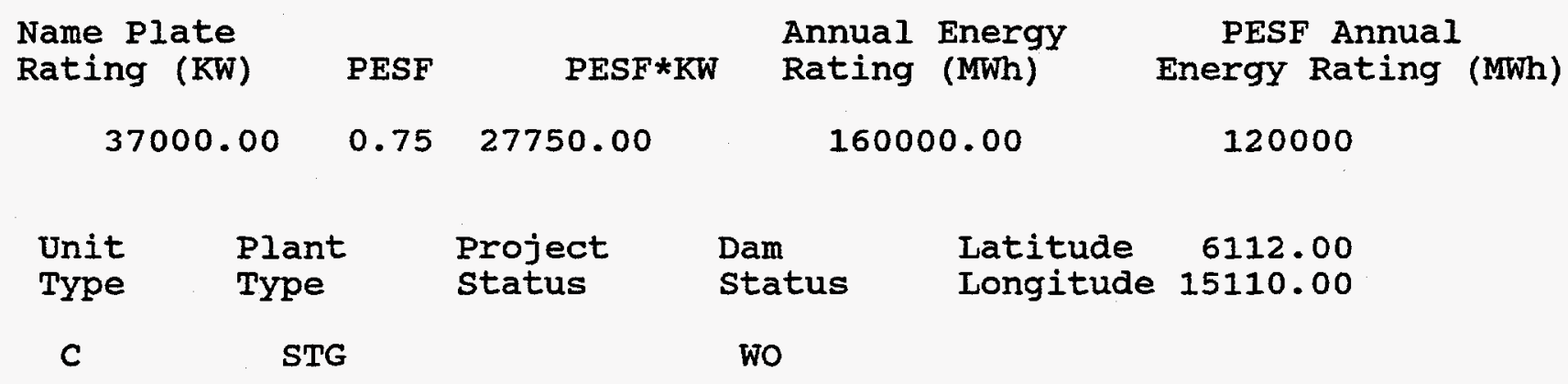

Factor

Exist Prob

Factor

Exist Prob

Wild/Scenic Protection

wild/Scenic Tributary or

Upstream/ Downstream

wild/Scenic Location

River Basin

CHAKACHATNA RIVER BASINS

Cultural value

Fish Presence Value

Geologic Value

Historic Value

other value

Recreation value

Scenic Value

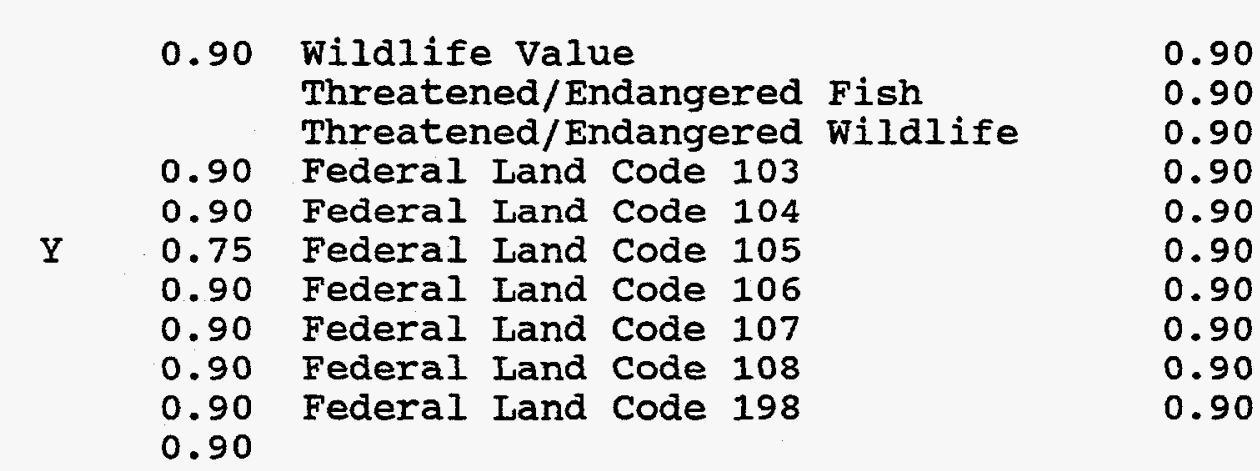


DATE: $10 / 31 / 97$

FERC

Number

Plant Name

AK014 UPPER BELUGA

County Name

MATANUSKA-SUSITNA DIV
PAGE NO: 71

State

Name

AK

Class Owner Name

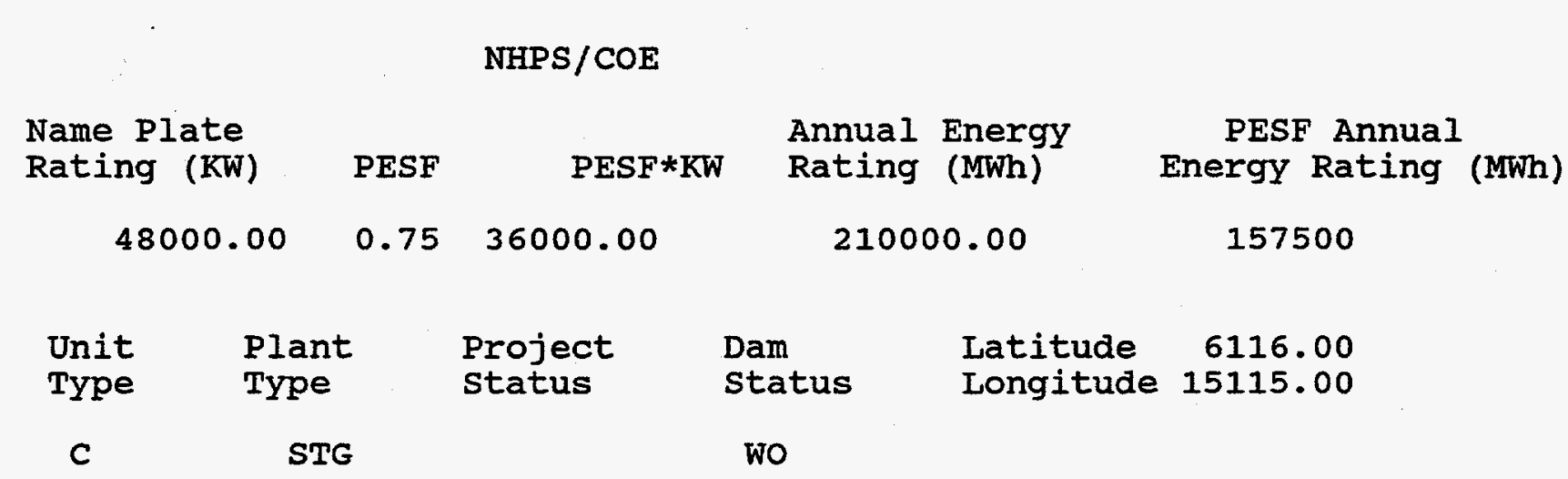

Factor

Exist Prob

Factor

Exist Prob

Wild/Scenic Protection

wild/Scenic Tributary or

Upstream / Downstream

wild/Scenic Location

BELUGA $\mathbf{R}$

Stream

River Basin

CHAKACHATNA RIVER BASINS

Cultural value

Fish Presence Value

Geologic Value

Historic Value

other value

Recreation Value

$\longrightarrow$

Factor

Exist Prob

Scenic Value

0.90 Wildlife Value

Threatened/Endangered Fish

Threatened/Endangered Wildlife

0.90 Federal Land Code 103

0.90 Federal Land Code 104

Y 0.75 Federal Land Code 105

0.90 Federal Land Code 106

0.90 Federal Land Code 107

0.90 Federal Land Code 108

0.90 Federal Land Code 198

0.90
0.90

0.90

0.90

0.90

0.90

0.90

0.90

0.90

0.90

0.90 
DATE: $10 / 31 / 97$

FERC

Number

Plant Name

AK015 YENTNA

County Name

MATANUSKA-SUSITNA DIV
PAGE NO: 72

state

Name

AK
River Basin

SUSITNA RIVER BASIN

class Owner Name

NHPS/COE

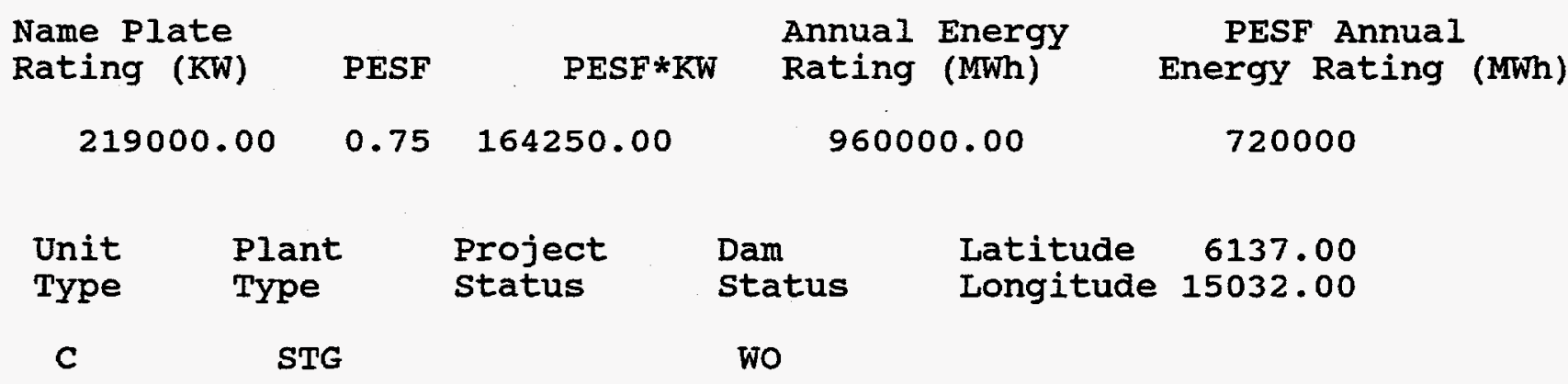

Factor

Exist Prob

Wild/Scenic Protection

wild/Scenic Tributary or

Upstream/Downstream

wild/Scenic Location

Cultural value

Fish Presence Value

Geologic Value

Historic Value

other value

Recreation Value

Scenic Value

$\mathrm{Y}$

$$
0.90
$$

wildife Value

Threatened/Endangered Fish

Threatened/Endangered Wildlife

0.90 Federal Land code 103

0.90 Federal Iand Code 104

0.75 Federal Land Code 105

0.90 Federal Land Code 106

0.90 Federal Land Code 107

0.90 Federal Land Code 108

0.90 Federal Land Code 198

0.90
Exist Prob

0.90

0.90

0.90

0.90

0.90

0.90

0.90

0.90

0.90

0.90 
DATE: $10 / 31 / 97$

FERC

Number

AK016
Plant Name

SKWENTNA (HAYES)

County Name

MATANUSKA-SUSITNA DIV
PAGE NO: 73

State

Name

SKWENTNA R

AK

\section{Class Owner Name}

NHPS/COE

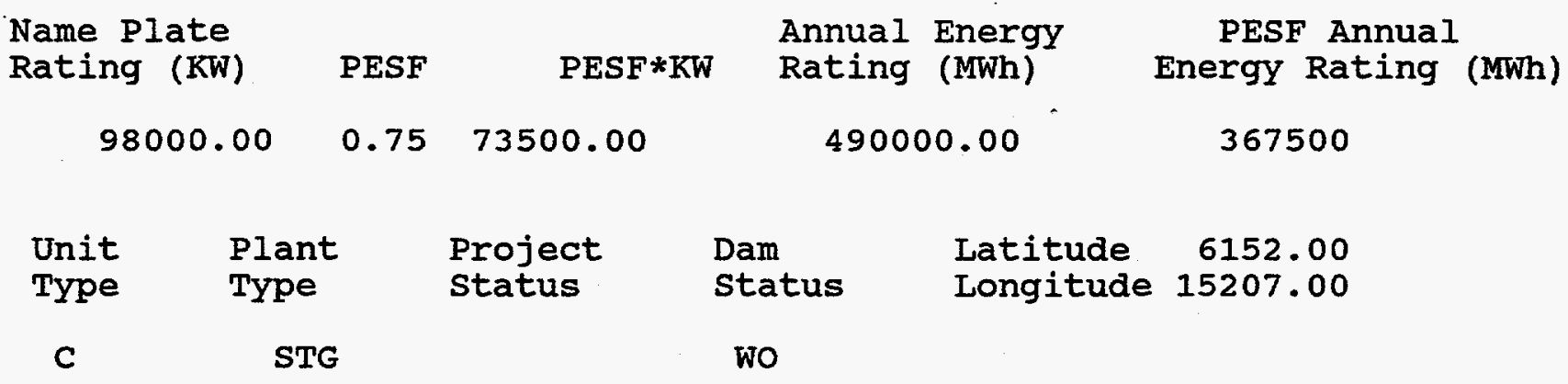

Factor

Exist Prob

wild/Scenic Protection

wild/Scenic Tributary or Upstream/Downstream wild/Scenic Location Cultural value Fish Presence Value Geologic Value Historic Value other Value Recreation Value Scenic Value
River Basin

SUSITNA RIVER BASIN 
DATE: $10 / 31 / 97$

FERC

Number

Plant Name

AK017 KEETNA (TALKEETNA)

County Name

MATANUSKA-SUSITNA DIV
PAGE NO: 74

state

Name

TALKEETNA $R$

AK
River Basin

SUSITNA RIVER BASIN

Class Owner Name

NHPS/COE

\begin{tabular}{|c|c|c|c|c|c|c|}
\hline $\begin{array}{l}\text { Name } \\
\text { Ratin }\end{array}$ & & PESF & PESF * KW & $\begin{array}{l}\text { Annual } \\
\text { Rating }\end{array}$ & $\begin{array}{l}\text { Energy } \\
\text { (MWh) }\end{array}$ & $\begin{array}{l}\text { PESF Annual } \\
\text { Energy Rating (MWh) }\end{array}$ \\
\hline & .00 & 0.75 & 55500.00 & 32400 & 00.00 & 243000 \\
\hline $\begin{array}{l}\text { Unit } \\
\text { Type }\end{array}$ & $\begin{array}{l}\text { Pl } \\
\text { Ty! }\end{array}$ & & $\begin{array}{l}\text { Project } \\
\text { Status }\end{array}$ & $\begin{array}{l}\text { Dam } \\
\text { status }\end{array}$ & $\begin{array}{l}\text { Latitude } \\
\text { Longitude }\end{array}$ & $\begin{array}{r}6227.00 \\
14942.00\end{array}$ \\
\hline C & & & & Wo & & \\
\hline
\end{tabular}

Factor

Wild/Scenic Protection

Wild/Scenic Tributary or

Upstream/Downstream

wild/Scenic Location

Cultural value

Fish Presence Value

Geologic Value

Historic Value

other Value

Recreation value

Scenic Value
Exist Prob

0.90

Wildlife Value

Threatened/Endangered Fish

Threatened/Endangered Wildlife

0.90 Federal Land Code 103

0.90 Federal Land Code 104

$Y \quad 0.75$ Federal Land code 105

0.90 Federal Land Code 106

0.90 Federal Land Code 107

0.90 Federal Land code 108

0.90 Federal Land Code 198
Exist Prob

0.90

0.90

0.90

0.90

0.90

0.90

0.90

0.90

0.90

0.90 
R E S O U R C E

DATE: $10 / 31 / 97$

FERC

Number

Plant Name

AK018 TALKEETNA 2

county Name

MATANUSKA-SUSITNA DIV

class

D A T A B A S E

L I S T I G

PAGE NO: 75

River Basin

SUSITNA RIVER BASIN

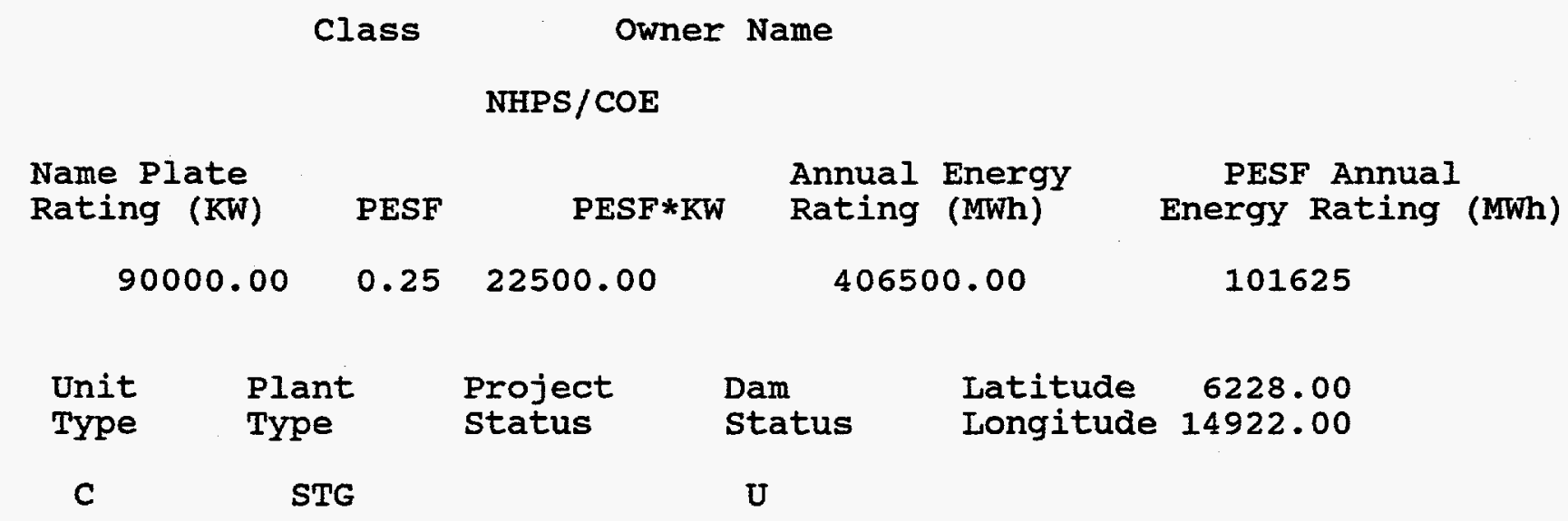

Factor

Exist Prob

Factor

Exist Prob

Wild/Scenic Protection

Wild/Scenic Tributary or

Upstream/Downstream

wild/Scenic Location

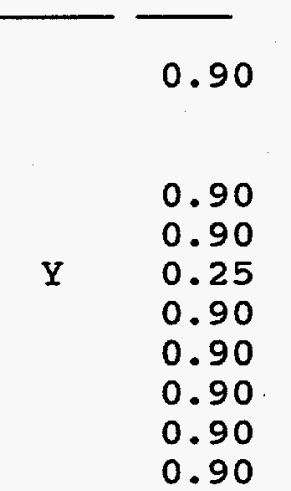

Wildlife Value

Threatened/Endangered Fish

Threatened/Endangered Wildlife

State

Name

AK

Cultural value

Fish Presence Value

Federal Land Code 103

Federal Land Code 104

Federal Land Code 105

Federal Land Code 106

Geologic Value

Federal Land Code 107

other Value

Federal Land Code 108

Recreation Value

Federal Land Code 198

0.90

0.90

0.90

0.90

0.90

0.90

0.90

0.90

0.90

Scenic Value

0.90
0.90

0.90 
DATE: $10 / 31 / 97$

FERC

Number

Plant Name

AK019 CHULITNA-HURRICANE

county Name

MATANUSKA-SUSITNA DIV
PAGE NO: 76

State

Name

CHULITNA R

AK

\section{Class Owner Name}

NHPS / COE

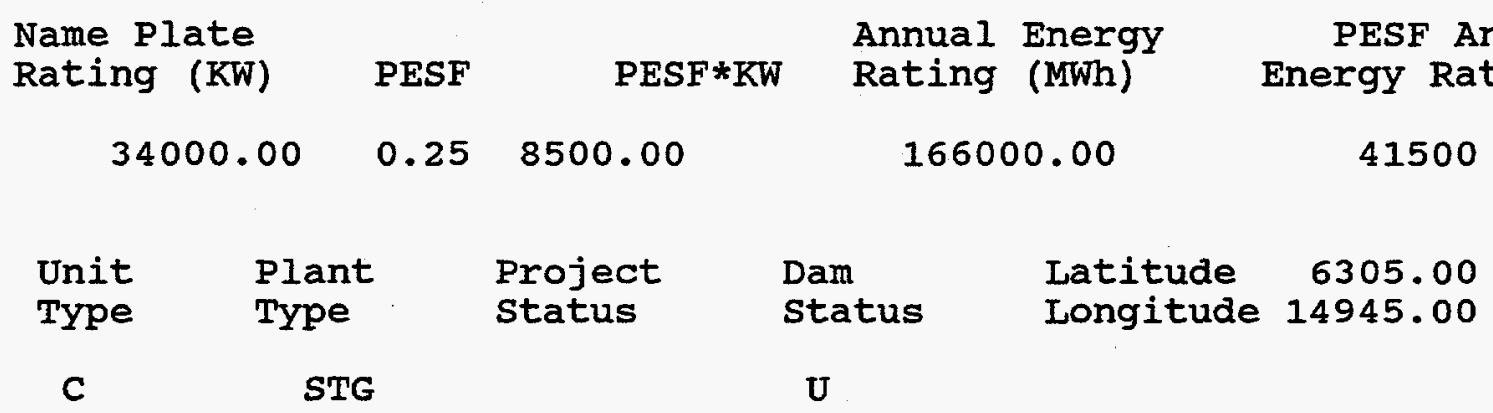

Factor

Exist Prob

Factor

Exist Prob

Wild/Scenic Protection

wild/Scenic Tributary or

Upstream/Downstream

wild/Scenic Location

Cultural value

Fish Presence Value

Geologic Value

River Basin

SUSITNA RIVER BASIN

Historic Value

other Value

Recreation Value

Scenic Value

$\begin{array}{clll} & & & \\ 0.90 & \begin{array}{l}\text { Wildlife Value } \\ \text { Threatened/Endangered Fish }\end{array} & 0.90 \\ & & 0.90 \\ & & \text { Threatened/Endangered Wildlife } & 0.90 \\ & 0.90 & \text { Federal Land Code 103 } & 0.90 \\ 0.90 & \text { Federal Land Code 104 } & 0.90 \\ 0.25 & \text { Federal Land Code 105 } & 0.90 \\ 0.90 & \text { Federal Land Code 106 } & 0.90 \\ 0.90 & \text { Federal Land Code 107 } & 0.90 \\ 0.90 & \text { Federal Land Code 108 } & 0.90 \\ 0.90 & \text { Federal Land Code 198 } & 0.90 \\ 0.90 & & \end{array}$


R E S O U R E E

DATE: $10 / 31 / 97$
D A T A B A S E

C

FERC

Number

Plant Name

AK020 SNOW

county Name

SEWARD DIVISION

Class

River Basin
PAGE NO: 77 state

Name

SNOW $\mathbf{R}$

AK

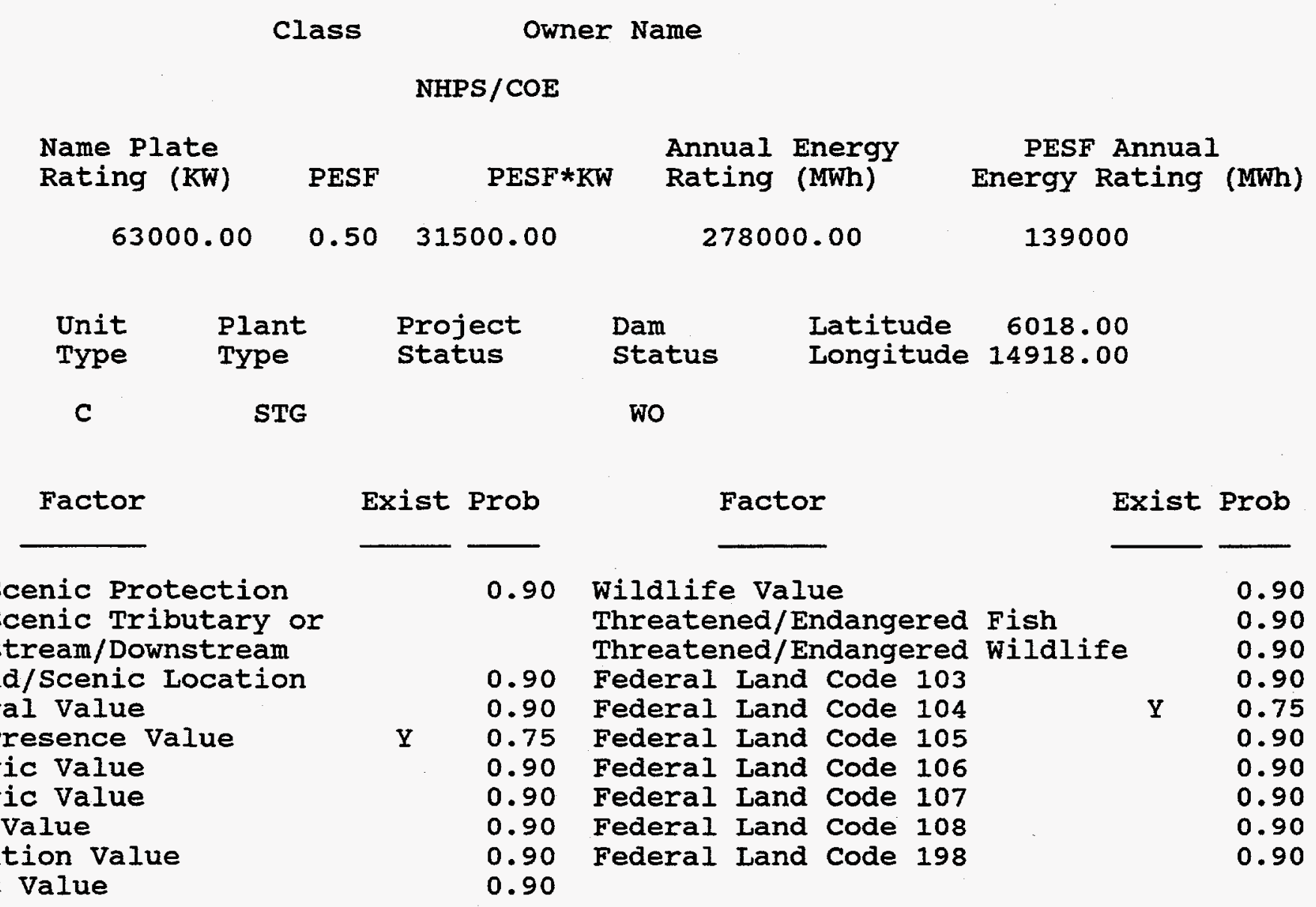

Wild/Scenic Protection

wild/Scenic Tributary or

Upstream/Downstream

wild/Scenic Location

Cultural value

Fish Presence Value

Geologic Value

Historic Value

other value

Recreation Value

Scenic Value

KENAI PENINSULA RIVER BAS 
DATE: $10 / 31 / 97$

PAGE NO: 78

FERC

Number

Plant Name

stream

State

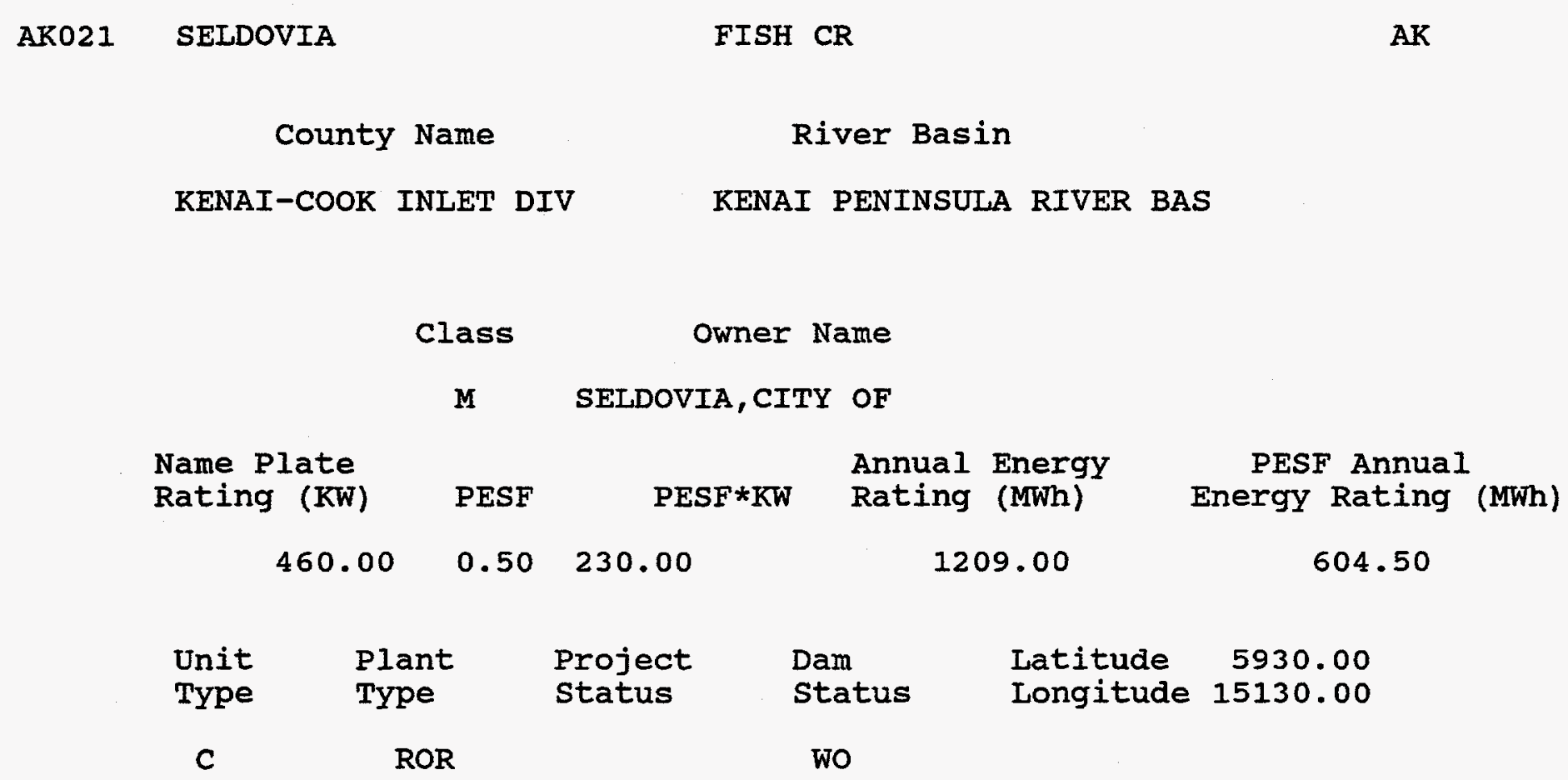

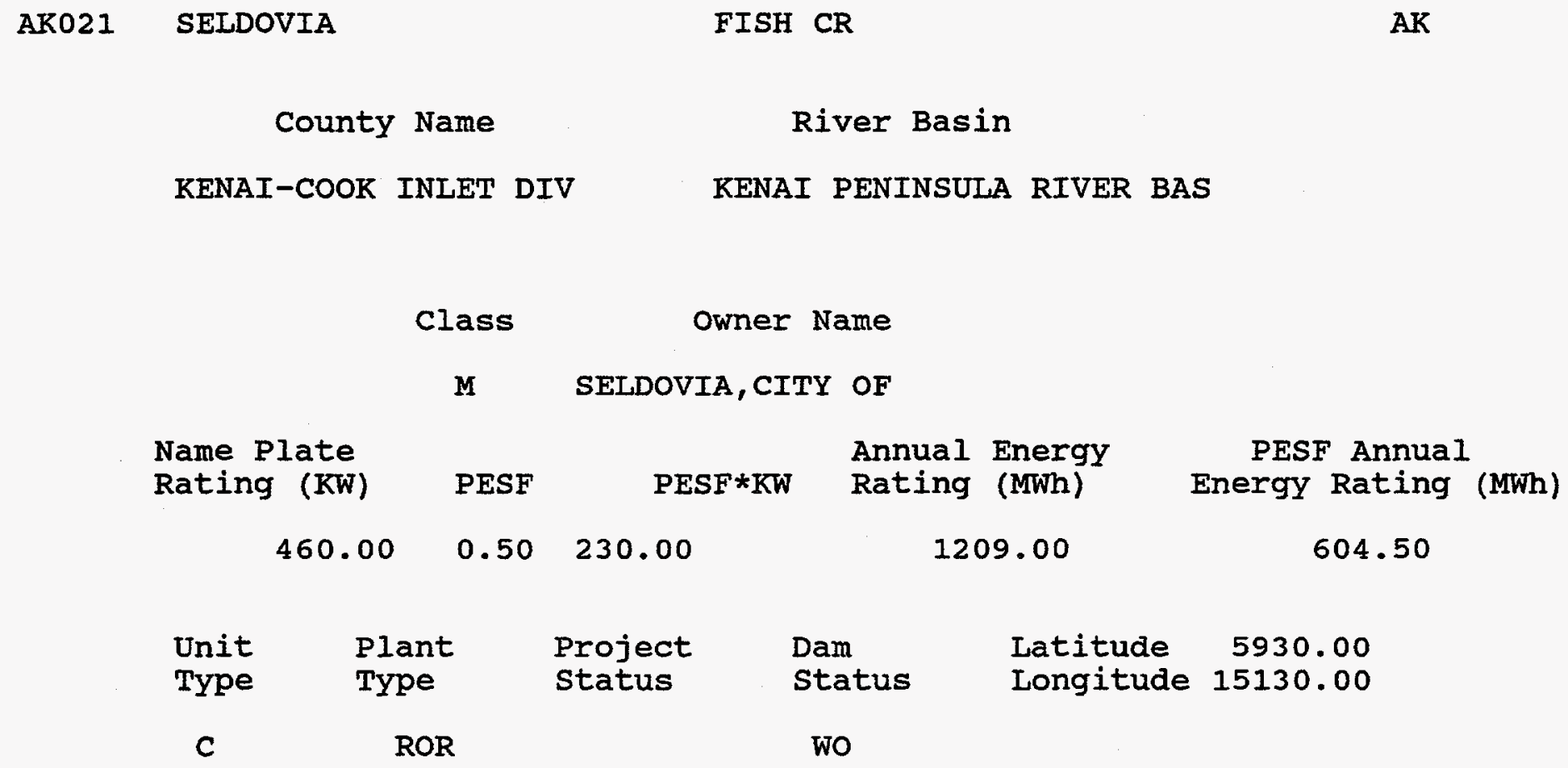

Name

Factor

Wild/Scenic Protection

wild/Scenic Tributary or Upstream/Downstream wild/Scenic Location Cultural value Fish Presence Value Geologic Value Historic Value other Value Recreation Value Scenic Value
Exist Prob

Factor

Exist Prob
0.90

0.90

0.90

0.90

Y $\quad 0.75$

0.90

0.90

0.90

0.90

0.90 
R E S O U R E

DATE: $10 / 31 / 97$

FERC

Number

AK022

BRADLEY LARE

Plant Name

D A T A B A S E

I I S I N G

PAGE NO: 79

$\begin{array}{lc}\text { stream } & \begin{array}{c}\text { State } \\ \text { Name }\end{array} \\ \text { BRADLEY CR } & \text { AK } \\ \text { River Basin } & \\ \text { KENAI PENINSULA RIVER BAS } & \end{array}$

$\begin{array}{cc}\text { Class } & \text { Owner Name } \\ \text { F } & \text { CORPS OF ENGINEERS }\end{array}$

$\begin{array}{rrrcc}\begin{array}{l}\text { Name Plate } \\ \text { Rating (KW) }\end{array} & \text { PESF } & \text { PESF*KW } & \begin{array}{c}\text { Annual Energy } \\ \text { Rating }\end{array} & \begin{array}{c}\text { PESF Annual } \\ \text { Energy Rating (MWh) }\end{array} \\ 63900.00 & 0.90 & 57510.00 & 335600.00 & 302040\end{array}$

\begin{tabular}{|c|c|c|c|c|}
\hline $\begin{array}{l}\text { Unit } \\
\text { Type }\end{array}$ & $\begin{array}{l}\text { Plant } \\
\text { Type }\end{array}$ & $\begin{array}{l}\text { Project } \\
\text { status }\end{array}$ & $\begin{array}{l}\text { Dam } \\
\text { status }\end{array}$ & $\begin{array}{lr}\text { Latitude } & 5945.00 \\
\text { Longitude } & 15057.00\end{array}$ \\
\hline C & STG & FA & W & \\
\hline
\end{tabular}

Factor

Exist Prob

Factor

Exist Prob

Wild/scenic Protection

wild/Scenic Tributary or

0.90 Wildlife Value

Threatened/Endangered Fish

Upstream/Downstream

wild/Scenic Location

Cultural Value

Fish Presence Value

Geologic Value

Threatened/Endangered wildlife

0.90 Federal Land Code 103

0.90 Federal Land Code 104

0.90 Federal Land Code 105

0.90 Federal Land Code 106

0.90 Federal Land Code 107

0.90 Federal Land Code 108

0.90 Federal Land Code 198

0.90
Other Value

Recreation Value

Scenic Value 
DATE: $10 / 31 / 97$

FERC

Number

Plant Name

AK023 LOWELL

County Name

SEWARD DIVISION

class

M SEWARD, CITY OF

Name Plate

Rating (KW)

PESF

Annual Energy

PESF*KW

Rating (MWh)

3000.00

$0.75 \quad 487.50$

Project

status

Unit
Type

Plant

Type

DIV
River Basin

KENAI PENINSULA RIVER BAS
LOWELI CR, DAY HARBOR
Exist Prob

Factor

Wild/Scenic Protection

Wild/Scenic Tributary or

Upstream/Downstream

wild/scenic Location

Cultural value

Fish Presence Value

Geologic Value

Historic Value

other Value

Recreation value

Scenic Value
PAGE NO: 80

state

Name

AK
PESF Annual Energy Rating (MWh)

2250

$\begin{array}{llr}\text { Dam } & \text { Latitude } & 6002.00 \\ \text { Status } & \text { Longitude } & 14909.00\end{array}$

พo

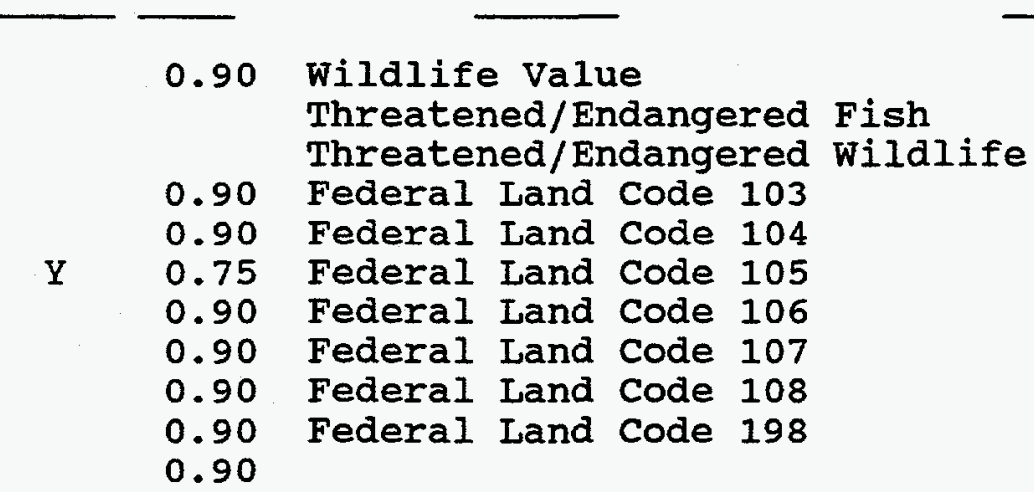

Exist Prob

Factor

0.90

0.90

0.90

0.90

0.90

0.90

0.90

0.90

0.90

0.90 
DATE: $10 / 31 / 97$

FERC

Number

Plant Name

AK024 MAIN IAAKE

County Name

VALDEZ-CHITINA-WHITT DV
PAGE NO: 81

State

Name

MAIN BAY,PRINCE WILIIAM SOUND AK

River Basin

KENAI PENINSULA RIVER BAS $\begin{array}{cc}\text { Class } & \text { Owner Name } \\ \text { M } & \text { ALASKA DEPT FISH \& GAME }\end{array}$

Name Plate

Rating (KW)

PESF

Annual Energy

$0.25 \quad 31.25$

PESF Annual

125.00

$\begin{array}{lll}\text { Unit } & \text { Plant } & \text { Project } \\ \text { Type } & \text { Type } & \text { Status }\end{array}$

C

Factor

Exist Prob
Wild/Scenic Protection

wild/Scenic Tributary or Upstream/Downstream wild/Scenic Location

Cultural Value

Fish Presence Value

Geologic Value

Historic Value

other Value

Recreation Value

Scenic Value
Rating (MWh)

400.00

PESF*KW

Factor

Latitude 6027.00

Dam

status

Longitude 14801.00

U Energy Rating (MWh)

100

Exist Prob

0.90 Wildlife Value

Threatened/Endangered Fish

Threatened/Endangered Wildlife

0.90

0.90

0.90

0.90 Federal Land Code 103

0.90 Federal Land Code 104

0.90

0.90

0.90

0.90

0.90

0.90

Federal Land Code 107

0.90

0.90 Federal Land Code 198

0.90 
DATE: $10 / 31 / 97$

FERC

Number

Plant Name

AK025 TAKATZ

county Name

SITKA DIVISION
PAGE NO: 82

state

Name

AK

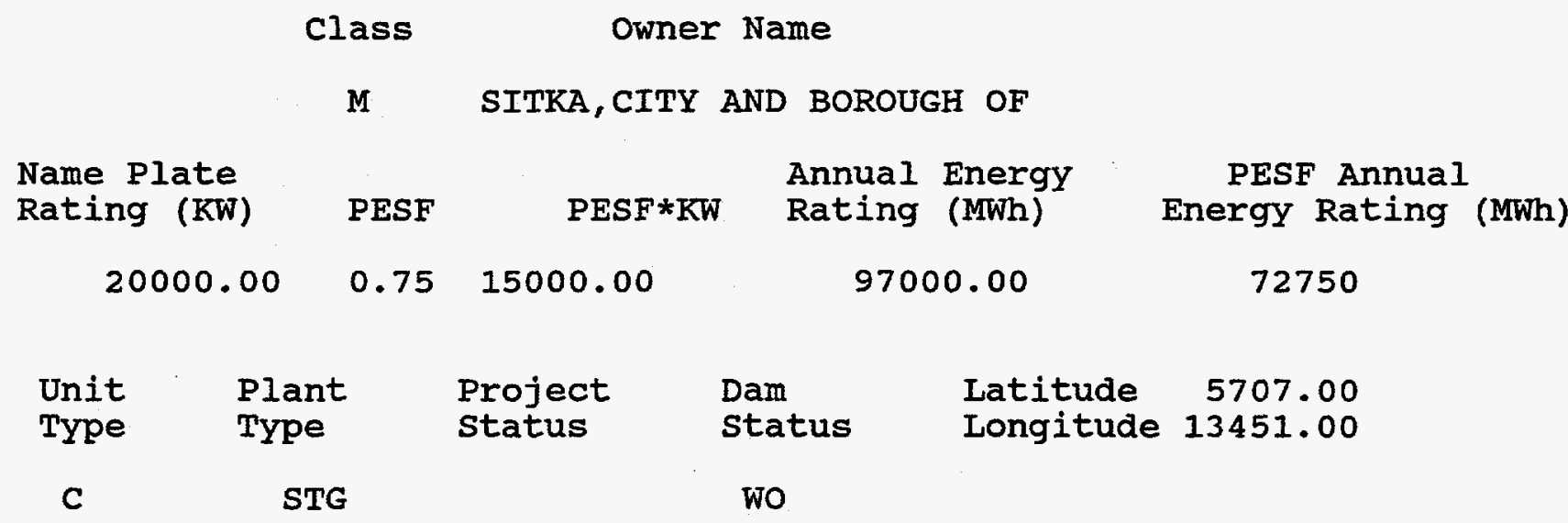

Factor

Exist Prob

Factor

Exist Prob

Wild/scenic Protection

Wild/Scenic Tributary or

Upstream/Downstream

wild/Scenic Location

TAKATZ CR, TAKATZ BAY

River Basin

JUNEAU GROUP

Cultural Value

Fish Presence Value

Geologic Value

Historic Value

other Value

Recreation Value

Scenic Value

0.90 Wildlife Value

Threatened/Endangered Fish

Threatened/Endangered Wildlife

0.90 Federal Land Code 103

0.90 Federal Land code 104

0.90 Federal Land Code 105

0.90 Federal Land Code 106

0.90 Federal Land Code 107

0.90 Federal Land Code 108

0.90 Federal Land Code 198

0.90
0.90

0.90

0.90

0.90

Y

0.75

0.90

0.90

0.90

0.90

0.90 
FERC

Number

AK026
Plant Name

KASNYKU LAKE

county Name

SITKA DIVISION

Class
State

Name

KASNYKU FALLS STR

AK
River Basin

JUNEAU GROUP

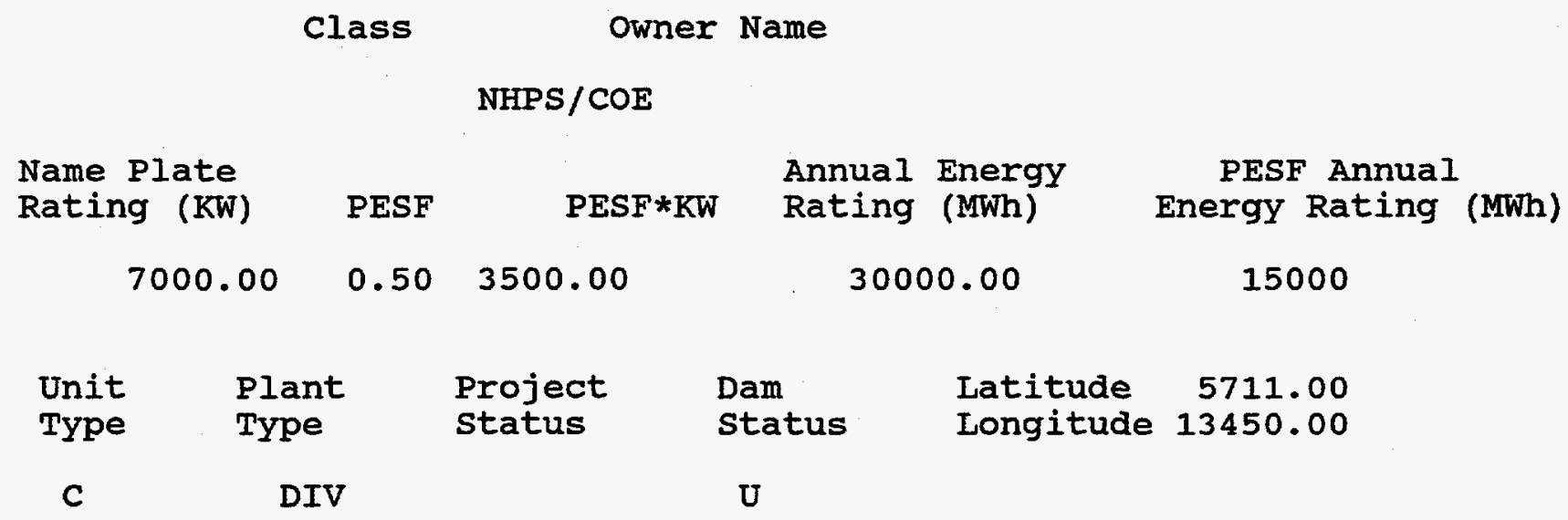

Factor

Exist Prob

Factor

Exist Prob

wild/Scenic Protection

wild/Scenic Tributary or

Upstream/Downstream wild/Scenic Location

Cultural value

Fish Presence Value

Geologic Value

Historic Value

other Value

Recreation Value

Scenic Value
0.90 Wildlife Value

Threatened/Endangered Fish Threatened/Endangered Wildlife

0.90 Federal Land Code 103

0.90 Federal Land Code 104

0.90 Federal Land Code 105

0.90 Federal Land Code 106

0.90 Federal Land Code 107

0.90 Federal Land Code 108

0.90 Federal Land Code 198
0.90

0.90

0.90

0.90

0.50

0.90

0.90

0.90

0.90

0.90 
DATE: $10 / 31 / 97$

FERC

Number

AK027

CARBON LAKE

County Name

SITKA DIVISION

Class
PAGE NO: 84

state

Name

CARBON LAKE

AK
River Basin

JUNEAU GROUP

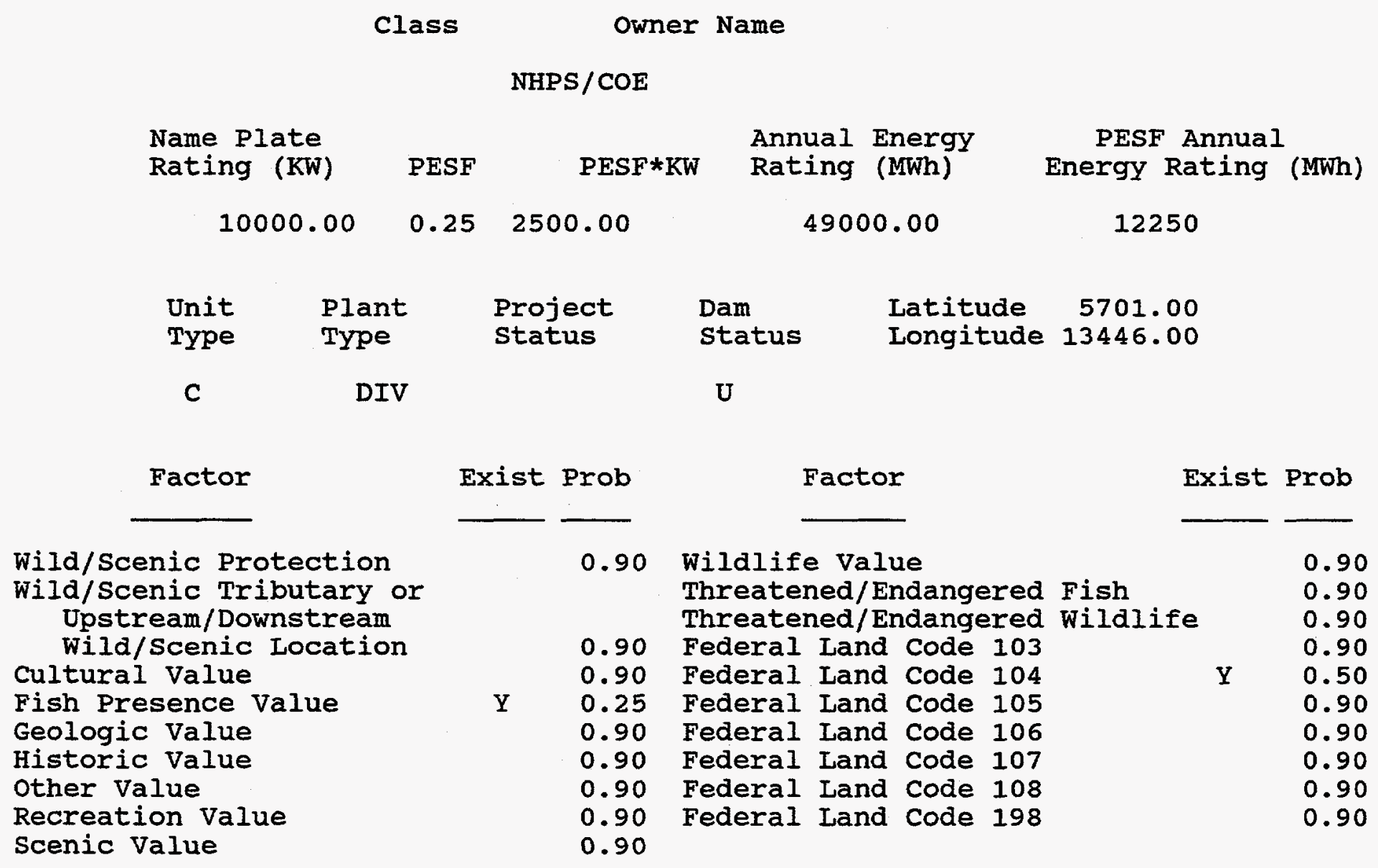


R E S O U R C E

DATE: $10 / 31 / 97$

FERC

Number

Plant Name

AKO28 MILK LAKE

County Name

SITKA DIVISION

Class

Owner Name

NHPS/COE

Name Plate

Rating ( $\mathrm{KW}$ )

PESF

PESF*KW

Annual Energy

7000.00

$0.25 \quad 1750.00$

Rating (MWh)

33000.00

I I S I N G

PAGE NO: 85

Stream

State

Name

AK
River Basin

JUNEAU GROUP

\begin{tabular}{|c|}
\hline $\begin{array}{l}\text { Unit } \\
\text { Type }\end{array}$ \\
\hline
\end{tabular}

C

DIV

$\mathrm{U}$

Factor

wild/scenic Protection

Wild/Scenic Tributary or

Upstream/Downstream

wild/scenic Location

Cultural value

Fish Presence Value

Geologic Value

Historic Value

other Value

Recreation Value

Scenic Value
Exist Prob

Factor

Exist Prob

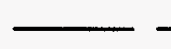

0.90







0.90
0.90
0.25
0.90
0.90
0.90
0.90
0.90

PESF Annual Energy Rating (MWh)

8250 
DATE: $10 / 31 / 97$

PAGE NO: 86

FERC

Number

Plant Name

Stream

State

AK029 BORODINO LAKE

BIG PORT WALTER, CHATHAM STRAIT

Name

County Name

SITKA DIVISION

River Basin

JUNEAU GROUP

Class Owner Name

NHPS/COE

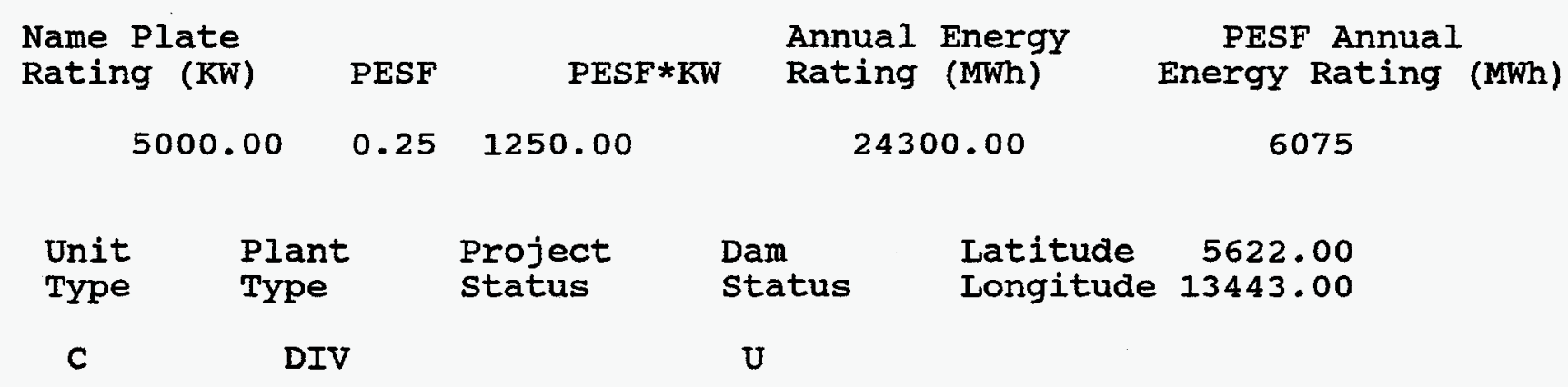

Factor

Exist Prob

Factor

Exist Prob

Wild/Scenic Protection

Wild/Scenic Tributary or

Upstream/Downstream

wild/Scenic Location

Cultural value

Fish Presence Value

-

0.90 Wildlife Value

0.90

Threatened/Endangered Fish

AK

Geologic Value

Threatened/Endangered Wildlife

0.90

0.90 Federal Land Code 103

0.90

0.90 Federal Land Code 104

0.90

$Y \quad 0.25$ Federal Land Code 105

0.50

0.90 Federal Land Code 106

0.90

0.90 Federal Land Code 107

0.90

0.90 Federal Land Code 108

0.90

other Value

Recreation value

0.90 Federal Land Code 198

0.90

0.90

0.90 
DATE : $10 / 31 / 97$

FERC

Number Plant Name

AK030 BETTY LAKE

County Name

SITKA DIVISION
PAGE NO: 87

State

Name

PORT ARMSTRONG, CHATHAM STRAIT

AK $\begin{array}{cc}\text { Class } & \text { Owner Name } \\ \text { M ALASKA DEPT FISH \& GAME }\end{array}$

Name Plate

Rating (KW)

PESF

Annual Energy

PESF*KW Rating (MWh)

700.00

$\begin{array}{llllr}\text { Unit } & \text { Plant } & \text { Project } & \text { Dam } & \text { Latitude } \\ \text { Type } & \text { Type } & \text { Status } & \text { Status } & \text { Longitude } 13441.00 \\ \text { C } & & & U\end{array}$

Factor

Exist Prob

Factor

Exist Prob

Wild/Scenic Protection

wild/scenic Tributary or

Upstream/Downstream

wild/Scenic Location

Cultural Value

Fish Presence Value

Geologic Value

Historic Value

other value

Recreation Value

Scenic Value
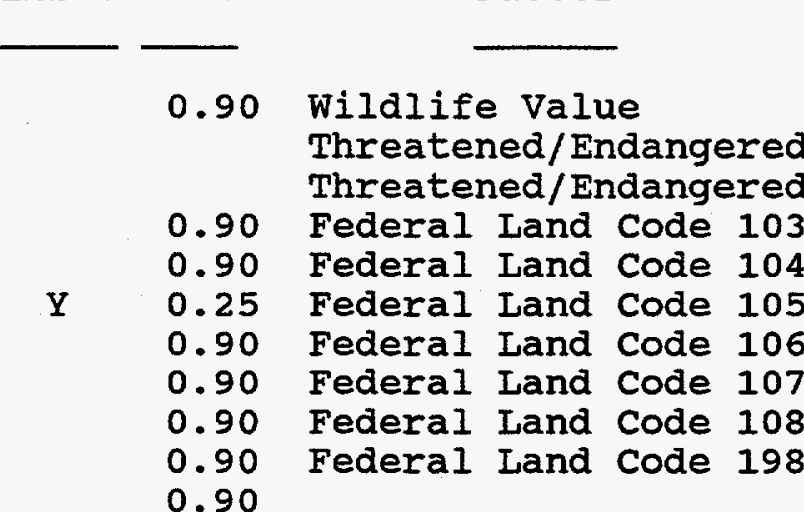

175

PESF Annual Energy Rating (MWh) 
DATE: $10 / 31 / 97$

PAGE NO: 88

FERC

Number

Plant Name

stream

State

AK031 MAKSOUTOF

MAKSOUTOF $\mathbf{R}$

Name

county Name

River Basin

SITKA DIVISION

JUNEAU GROUP

Class

Owner Name

NHPS / COE

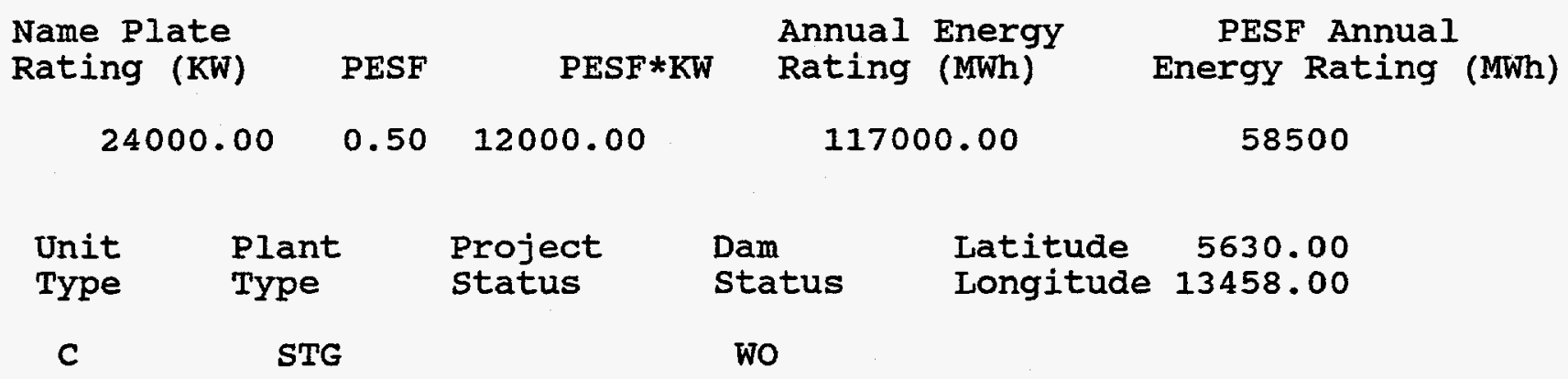

Factor

Wild/Scenic Protection

wild/Scenic Tributary or Upstream/Downstream wild/scenic Location Cultural value Fish Presence Value Geologic Value Historic Value other value

Recreation value

Scenic Value
Exist Prob
Exist Prob

Factor

AK 
DATE: $10 / 31 / 97$

FERC

Number

AK032

DIANA LAKE

County Name

SITKA DIVISION

Class

Owner Name

NHPS / COE

Name Plate

Rating (KW)

PESF

PESF*KW

Annual Energy

8000.00

0.252000 .00

35000.00

Latitude $\quad 5653.00$

Unit Plant

Type

Type

Project status

PAGE NO: 89
River Basin

JUNEAU GROUP state

Name

AK

\author{
C \\ DIV \\ Factor \\ wild/Scenic Protection \\ wild/Scenic Tributary or \\ Upstream/Downstream \\ wild/Scenic Location \\ Cultural value \\ Fish Presence Value \\ Geologic Value \\ Historic Value \\ other Value \\ Recreation Value \\ Scenic Value
}

Exist Prob

Factor

Exist Prob

\begin{tabular}{ll}
\hline & \\
& \\
& \\
& \\
& \\
& 0.90 \\
& 0.90 \\
0.90 \\
0.25 \\
0.90 \\
0.90 \\
0.90 \\
0.90 \\
0.90
\end{tabular}

\section{wildlife Value}

Threatened/Endangered Fish Threatened/Endangered Wildlife

Federal Land Code 103

Federal Land Code 104

Federal Land Code 105

Federal Land code 106

Federal Land Code 107

Federal Land Code 108

Federal Land Code 198
0.90

0.90

0.90

0.90

0.90

0.50

0.90

0.90

0.90

0.90

0.90
PESF Annual

Energy Rating (MWh)

8750 
DATE : $10 / 31 / 97$

PAGE NO: 90

FERC

Number

Plant Name

stream

State

AK033 SUPERIOR

ELFIN COVE(WACHUSETT COVE CR)

Name

AK

county Name

River Basin

SKAGWAY-YAKUTAT DIV

JUNEAU GROUP

Class Owner Name

I SUPERIOR PACKING CO

Name Plate

Rating (KW)

750.00

PESF

Annual Energy

PESF*KW Rating (MWh)

3300.00

$0.75 \quad 562.50$

Project

status

Type

Plant

Type

Exist Prob

Factor

Latitude $\quad 5747.00$

status

Longitude 13505.00

Wo
PESF Annual Energy Rating (MWh)
ROR

Factor

C

Factor

Wild/Scenic Protection Wild/Scenic Tributary or Upstream/Downstream wild/Scenic Location Cultural value

Fish Presence Value

Geologic Value

Historic Value

other Value

Recreation Value

Scenic Value
0.90 Wildlife Value Threatened/Endangered Fish Threatened/Endangered Wildlife
0.90 Federal Land Code 103
0.90 Federal Land Code 104
0.90 Federal Land Code 105
0.90 Federal Land Code 106
0.90 Federal Land Code 107
0.90 Federal Land Code 108
0.90 Federal Land Code 198
0.90

Exist Prob

0.90

0.90

0.90

0.90

0.75

0.90

0.90

0.90

0.90

0.90 
DATE: $10 / 31 / 97$

PAGE NO: 91

FERC

Number

AK034 PELICAN

County Name

SKAGWAY-YAKUTAT DIV

Plant Name class

P

PELICAN UTILITY CO

Name Plate Rating (KW)

PESF

PESF*KW

Annual Energy Rating (MWh)

1445.00

$$
1000.00
$$

0.25250 .00 stream

PELICAN CR,LISIANSKI INLET

River Basin

JUNEAU GROUP
State

Name

AK

Project

status

Type

$$
\begin{aligned}
& \text { Plant } \\
& \text { Type } \\
& \text { ROR }
\end{aligned}
$$

Factor

Exist Prob

Factor

Exist Prob

Wild/Scenic Protection

Wild/Scenic Tributary or

Upstream / Downstream

wild/Scenic Location

Cultural value

Fish Presence Value

Geologic Value

Historic Value

other value

Recreation value

Scenic Value

$\begin{array}{llr}\text { Dam } & \text { Latitude } & 5757.00 \\ \text { Status } & \text { Longitude } & 13614.00\end{array}$

U
PESF Annual Energy Rating (MWh)

361.25

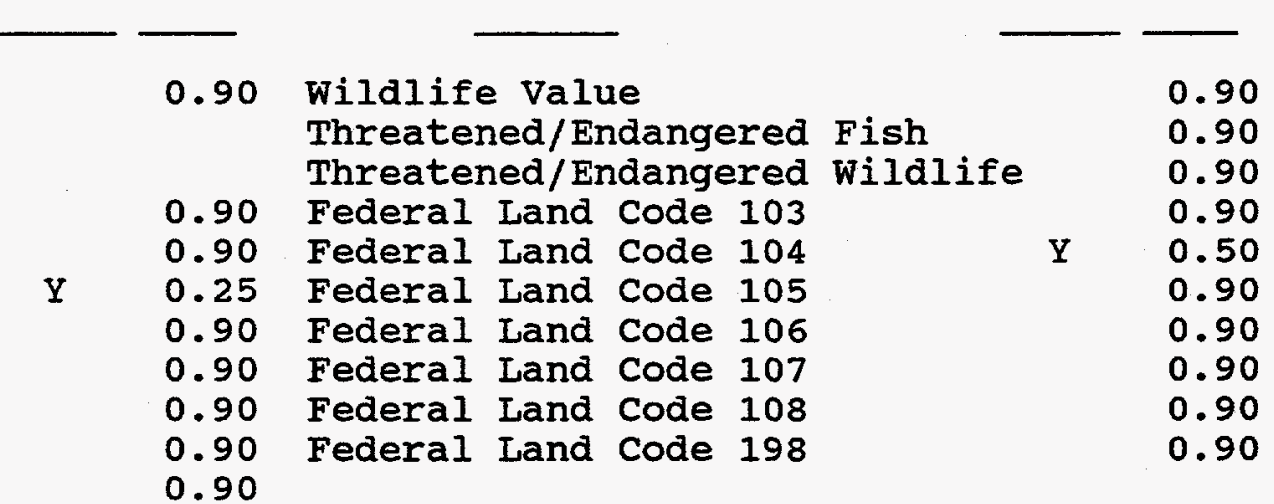


FERC

Number

AK035 GOLD CREEK

County Name

JUNEAU DIVISION
Plant Name

Class

Owner Name

P ALASKA ELEC LT \& PWR CO

Name Plate

Rating (KW)

PESF

Annual Energy

PESF*KW Rating (MWh)

8970.00

$0.90 \quad 1800.00$

JUNEAU GROUP

stream

State

Name

AK

$$
2000.00
$$

0.90

$\begin{array}{cc}\text { Unit } & \text { Plant } \\ \text { Type } & \text { Type } \\ \text { C } & \text { DIV }\end{array}$

\section{Project} status

DIV
Factor

Wild/Scenic Protection

wild/Scenic Tributary or Upstream/Downstream wild/Scenic Location Cultural Value

Fish Presence Value Geologic Value Historic Value other Value Recreation value Scenic Value
Dam
status

U
Exist Prob

0.90

Wildlife Value Threatened/Endangered Fish Threatened/Endangered Wildiife

Latitude $\quad 5818.00$ Longitude 13424.00
PESF Annual Energy Rating (MWh)

8073

0.90 Federal Land Code 103

0.90 Federal Land Code 104

0.90 Federal Land Code 105

0.90 Federal Land Code 106

0.90 Federal Land Code 107

0.90 Federal Land Code 108

0.90 Federal Land Code 198

Exist Prob

0.90

0.90

0.90

0.90

0.90

0.90

0.90

0.90

0.90

0.90

0.90 

R E S O U R C E
D A T A B A S E
I I S T I N G

DATE: $10 / 31 / 97$

PAGE NO: 93

FERC

Number

Plant Name

Stream

State

AK036 ANNEX CREEK

ANNEX CR, TAKU INLET

Name

AK

county Name

JUNEAU DIVISION
River Basin

JUNEAU GROUP $\begin{array}{cc}\text { Class } & \text { Owner Name } \\ \text { P } & \text { ALASKA ELEC LT \& PWR CO }\end{array}$

Name Plate

Rating ( $\mathrm{KW}$ )

PESF

Annual Energy

PESF*KW Rating (MWh)

3000.00

Unit Plant Project

Type Type Status

c

DIV

Factor

Exist Prob

Factor

Exist Prob

Wild/Scenic Protection

wild/Scenic Tributary or

Upstream/Downstream

wild/Scenic Location

Cultural value

Fish Presence Value

Geologic Value

Historic Value

other value

Recreation value

Scenic Value

\section{Latitude $\quad 5820.00$ \\ Dam \\ Status \\ Longitude 13408.00}

wo
PESF Annual Energy Rating (MWh)

1500 
FERC

Number

AK037
Plant Name

LAKE DOROTHY

County Name

JUNEAU DIVISION

Class
Stream

DOROTHY CR

River Basin

JUNEAU GROUP
State

Name

AK

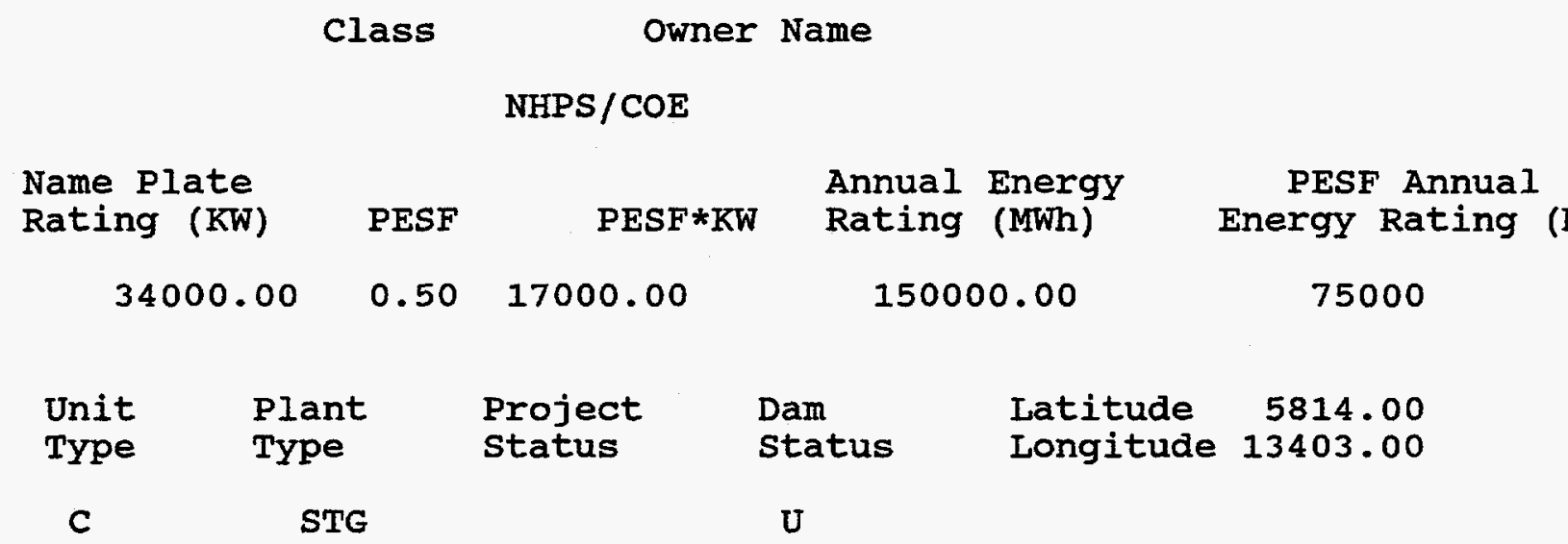

Factor

Exist Prob

Factor

Exist Prob

Wild/Scenic Protection

Wild/Scenic Tributary or

Upstream/Downstream

0.90 Wildlife Value

0.90

Threatened/Endangered Fish

wild/Scenic Location Threatened/Endangered Wildlife

0.90

0.90 Federal Land Code 103

0.90

Cultural Value

0.90 Federal Land Code 104

0.90

Fish Presence Value

0.90 Federal Land Code 105

0.50

0.90 Federal Land code 106

0.90

Geologic Value

0.90 Federal Land Code 107

0.90

Historic Value

0.90 Federal Land Code 108

0.90

other Value

Recreation Value

0.90 Federal Land Code 198

0.90

0.90

0.90 
DATE: $10 / 31 / 97$

FERC

Number

AK038
Plant Name

SPEEL DIVISION

County Name

JUNEAU DIVISION

Class
PAGE NO: 95

State

Name

SPEEL $\mathbf{R}$

AK
River Basin

JUNEAU GROUP

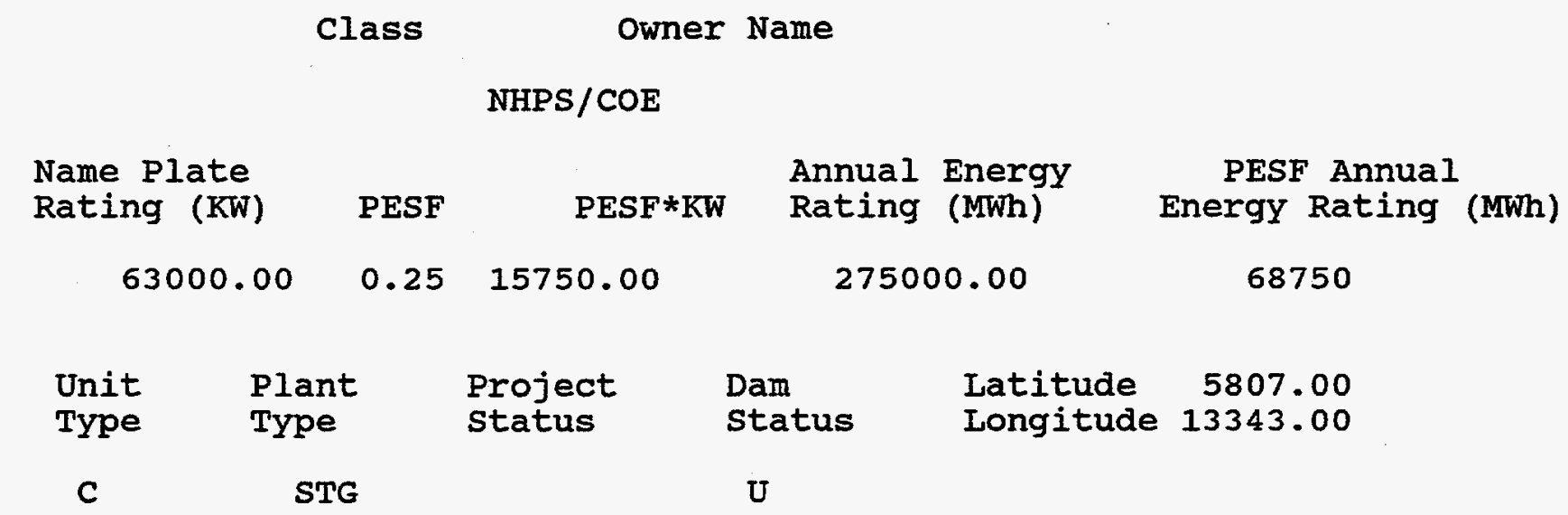

Factor

Wild/Scenic Protection

wild/Scenic Tributary or

Upstream/Downstream

wild/Scenic Location

Cultural value

Fish Presence Value

Geologic Value

Historic Value

other value

Recreation value

Scenic Value
Exist Prob

Factor

Exist Prob

$\begin{array}{cc} & \\ & 0.90 \\ & \\ & \\ & 0.90 \\ & 0.90 \\ & 0.25 \\ 0.90 \\ 0.90 \\ 0.90 \\ 0.90 \\ 0.90\end{array}$

wildlife Value Threatened/Endangered Fish Threatened/Endangered Wildlife Federal Land code 103 Federal Land Code 104 Federal Land Code 105 Federal Land Code 106 Federal Land Code 107 Federal Land Code 108 Federal Land Code 198

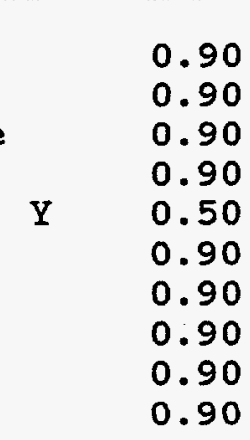


DATE: $10 / 31 / 97$

FERC

Number

Plant Name

AK039 CRATER LAKE

county Name

JUNEAU DIVISION

class

Owner Name

NHPS/COE

Name Plate

Rating (KW)

PESF

PESF*KW

Annual Energy

27000.00

0.25

6750.00
River Basin

JUNEAU GROUP
106000.00
PAGE NO: 96

State

Name

AK

$\begin{array}{cclll}\text { Unit } & \text { Plant } & \text { Project } & \text { Dam } & \text { Latitude } \\ \text { Type } & \text { Type } & \text { Status } & \text { Status } & \text { Longitude } 13346.00 \\ \text { C } & \text { STG } & & \text { U } & \end{array}$

Factor

wild/Scenic Protection

Wild/Scenic Tributary or Upstream/Downstream wild/Scenic Location Cultural value Fish Presence value Geologic Value Historic Value other Value Recreation value Scenic Value
Exist Prob

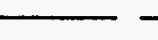

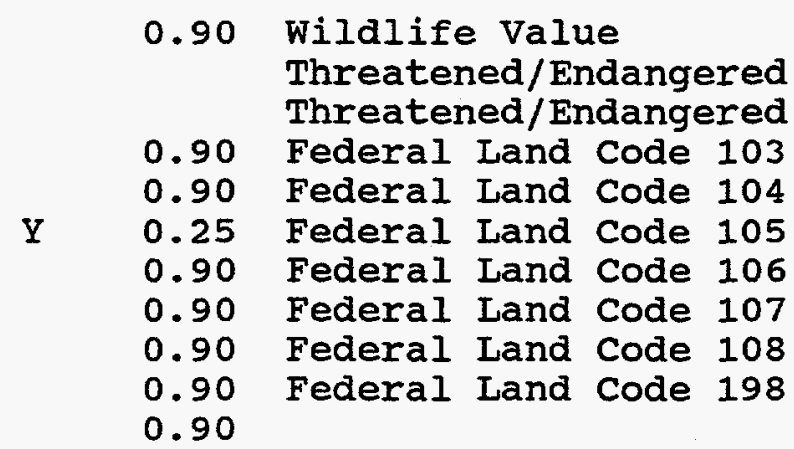

Factor
PESF Annual Energy Rating (MWh)

\section{0}

Exist Prob

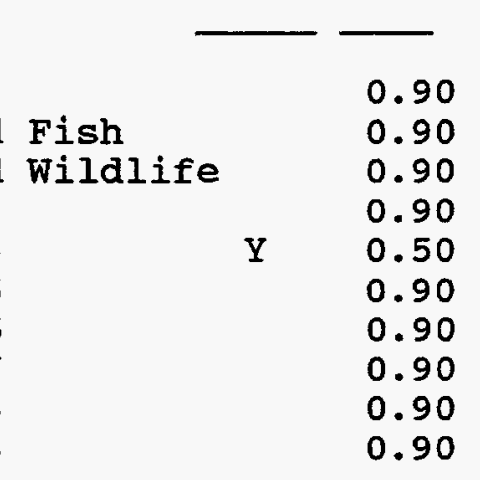


DATE: $10 / 31 / 97$

FERC

Number

Plant Name

AK040 TEASE

County Name

JUNEAU DIVISION

Class

Owner Name

F ALASKA POWER ADMIN

Name Plate

Rating (KW)

PESF

$$
\mathrm{PESF} * \mathrm{KW}
$$

Annual Energy Rating (MWh)

PAGE NO: 97

$$
16000.00 \quad 0.75 \quad 12000.00
$$

$$
70000.00
$$

Stream

State

Name

\begin{tabular}{|c|c|c|c|c|}
\hline $\begin{array}{l}\text { Unit } \\
\text { Type }\end{array}$ & $\begin{array}{l}\text { Plant } \\
\text { Type }\end{array}$ & $\begin{array}{l}\text { Project } \\
\text { status }\end{array}$ & $\begin{array}{l}\text { Dam } \\
\text { status }\end{array}$ & $\begin{array}{lr}\text { Latitude } & 5810.00 \\
\text { Longitude } & 13344.00\end{array}$ \\
\hline C & RES & & wo & \\
\hline
\end{tabular}

AK

52500
Factor

Wild/Scenic Protection

Wild/Scenic Tributary or Upstream/Downstream wild/Scenic Location Cultural value Fish Presence Value Geologic Value Historic Value other value Recreation Value Scenic Value
Exist Prob

0.90

wildlife Value Threatened/Endangered Fish Threatened/Endangered Wildlife

0.90 Federal Land Code 103

0.90 Federal Land Code 104

0.90 Federal Land Code 105

0.90 Federal Land Code 106

0.90 Federal Land Code 107

0.90 Federal Land Code 108

0.90 Federal Land Code 198
PESF Annual Energy Rating (MWh)
Exist Prob

0.90

0.90

0.90

0.90

$\mathrm{Y}$

0.75

0.90

0.90

0.90

0.90

0.90

0.90 
R E S O U R C E

DATE: $10 / 31 / 97$
D A T A B A S E

L I S T I N G

PAGE NO: 98

FERC

Number

Plant Name

Stream

State

Name

AK041 SWEETHEART FALLS

SWEETHEART FALLS CR

AK

County Name

River Basin

JUNEAU DIVISION

JUNEAU GROUP

class

Owner Name

NHPS/COE

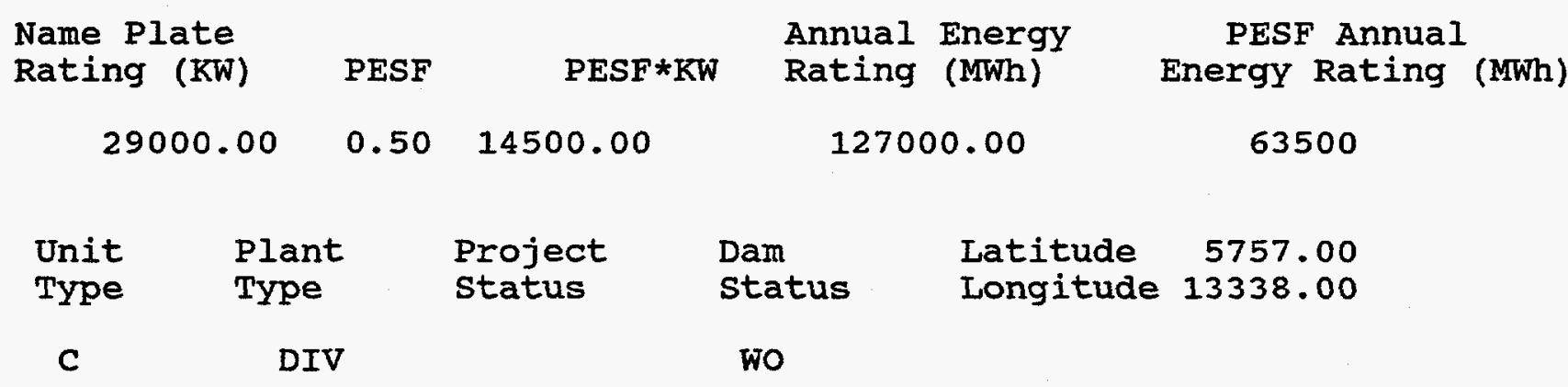

Factor

Exist Prob

Factor

Exist Prob

wild/Scenic Protection

wild/Scenic Tributary or

0.90 Wildlife Value

Threatened/Endangered Fish

Upstream/Downstream

wild/Scenic Location

Cultural value

Fish Presence Value

Threatened/Endangered Wildlife

0.90 Federal Land Code 103

0.90 Federal Iand Code 104

$\mathrm{Y} \quad 0.75$ Federal Land Code 105

$\begin{array}{lll}0.90 & \text { Federal Land Code } 106 \\ 0.90 & \text { Federal Land Code } 107\end{array}$

Historic Value

0.90 Federal Land Code 107
0.90 Federal Land Code 108

0.90 Federal Land Code 198

$\begin{array}{ll}\text { Recreation Value } & 0.90 \\ \text { Scenic Value } & 0.90\end{array}$

0.90

0.90

0.90

0.90

0.75

0.90

0.90

0.90

0.90

0.90 
DATE : $10 / 31 / 97$

FERC

Number

AK042 UPPER SWEETHEART

County Name

JUNEAU DIVISION

Class

PAGE NO: 99

Stream

SWEETHEART FALLS CR

River Basin

JUNEAU GROUP state

Name

AK

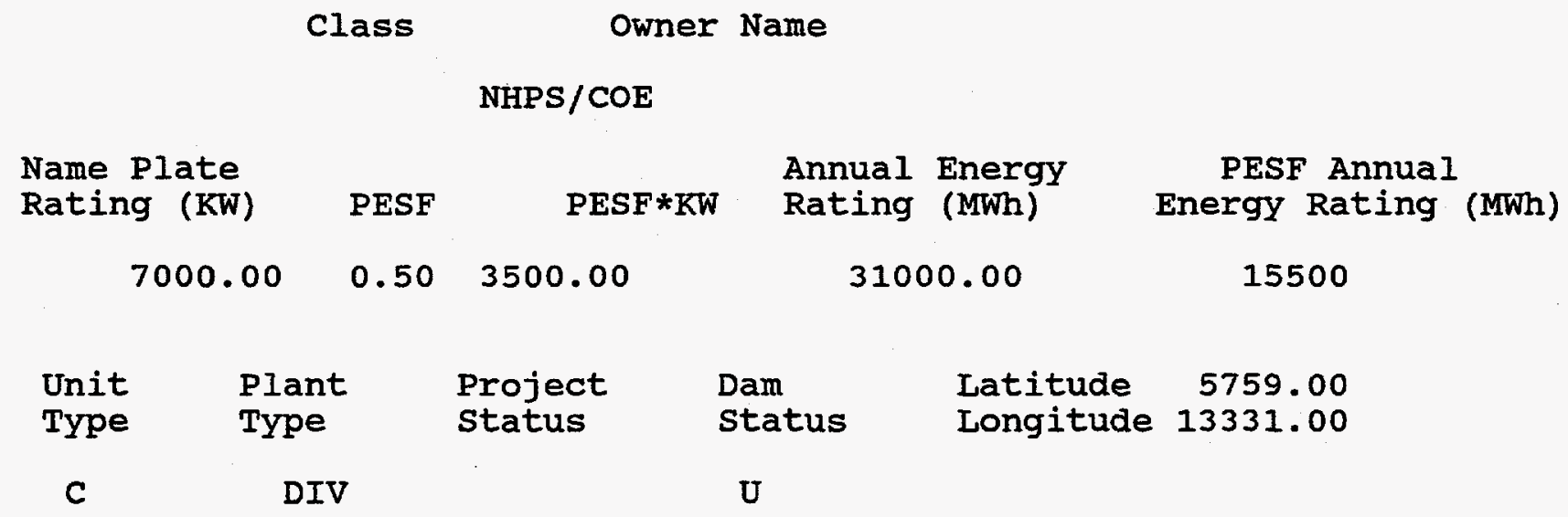

Factor

Exist Prob

Factor

Exist Prob

Wild/Scenic Protection

Wild/Scenic Tributary or

Upstream/Downstream

wild/Scenic Location

Cultural Value

Fish Presence Value

Geologic Value

Historic Value

other Value

Recreation value

Scenic Value

0.90 Wildlife Value Threatened/Endangered Fish Threatened/Endangered Wildlife

0.90 Federal Land Code 103

0.90 Federal Land Code 104

0.90 Federal Land Code 105

0.90 Federal Land Code 106

0.90 Federal Land Code 107

0.90 Federal Land Code 108

0.90 Federal Land Code 198
Exist Prob

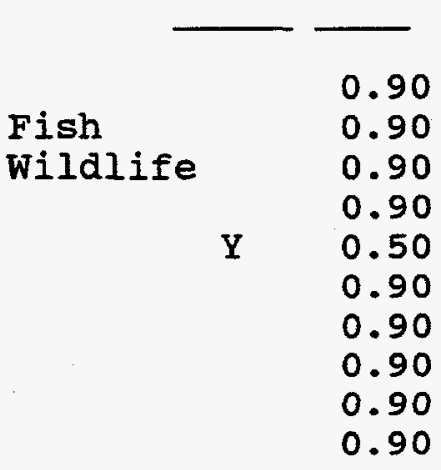


DATE: $10 / 31 / 97$

PAGE NO: 100

FERC

Number

Plant Name

stream

state

AK043 SCENERY

SCENERY CR

Name

AK

County Name

River Basin

WRANGELI-PETERSBURG DIV JUNEAU GROUP

Class Owner Name

NHPS/COE

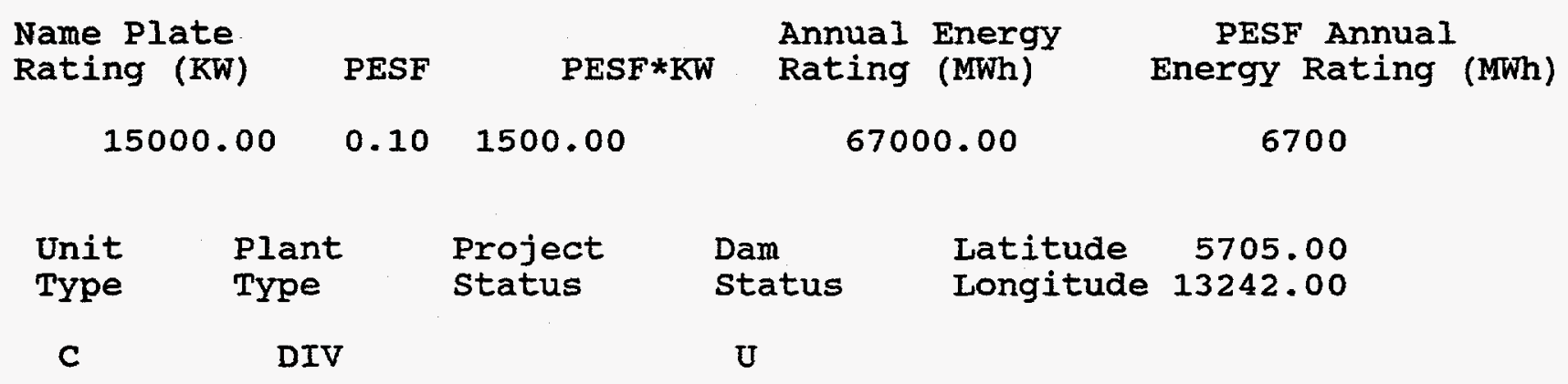

Factor

Exist Prob

Factor

Exist Prob

Wild/scenic Protection

wild/Scenic Tributary or

Upstream/Downstream

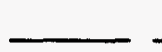

wild/scenic Location

Cultural Value

Fish Presence Value

Geologic Value

Historic Value

other Value

Recreation Value

Scenic Value

$\begin{array}{lll} & 0.90 & \text { Wildife Value } \\ & & \text { Threatened/Endangered Fish } \\ & & \text { Threatened/Endangered Wildlife } \\ & 0.90 & \text { Federal Land Code } 103 \\ & 0.90 & \text { Federal Land Code } 104 \\ \text { Y } & 0.25 & \text { Federal Land Code } 105 \\ & 0.90 & \text { Federal Land Code } 106 \\ & 0.90 & \text { Federal Land Code } 107 \\ & 0.90 & \text { Federal Land Code } 108 \\ \text { Y } & 0.25 & \text { Federal Land Code } 198 \\ Y & 0.50 & \end{array}$

0.90

0.90

0.90

0.90

$\mathrm{Y}$

0.50

0.90

0.90

0.90

0.90

0.90 
DATE: $10 / 31 / 97$

FERC

Number

Plant Name

AK044 RUTH LAKE

County Name

WRANGELL-PETERSBURG DIV
PAGE NO: 101

State

Name

AK

\section{Class Owner Name}

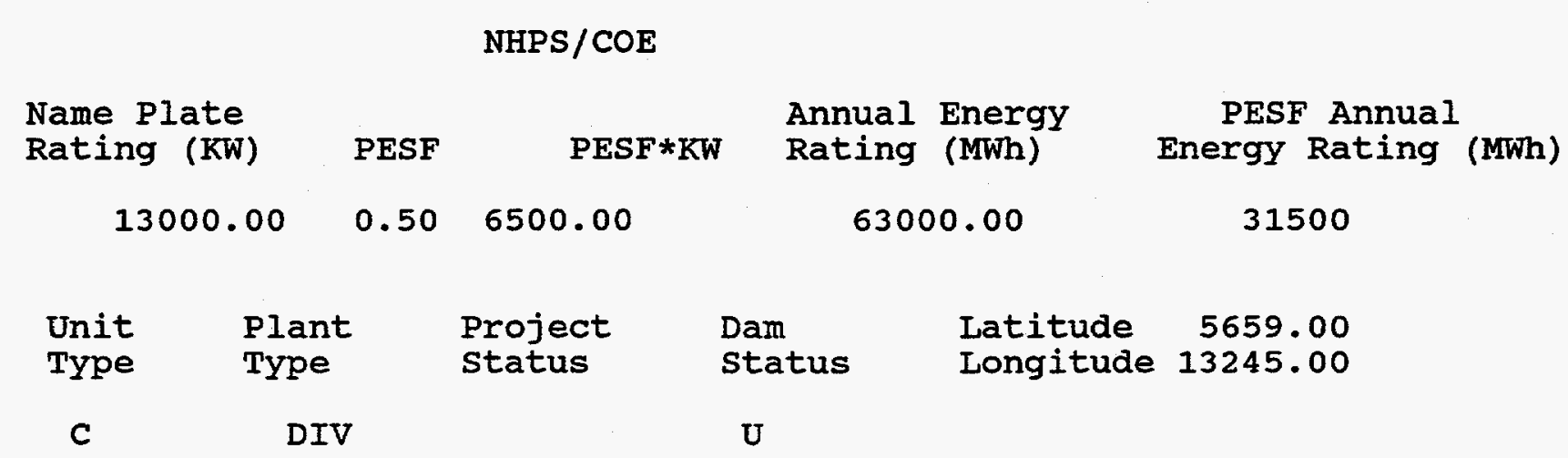

Factor

Exist Prob

Factor

Exist Prob

Wild/Scenic Protection

wild/Scenic Tributary or Upstream/ Downstream wild/Scenic Location

DELTA CR

stream

River Basin

JUNEAU GROUP

cultural value

Fish Presence Value

Geologic Value

Historic Value

other value

Recreation Value

Scenic Value

0.90 Wildlife Value

Threatened/Endangered Fish

Threatened/Endangered Wildlife

0.90 Federal Land Code 103

0.90 Federal Land Code 104

0.90 Federal Land code 105

0.90 Federal Land Code 106

0.90 Federal Land Code 107

0.90 Federal Land Code 108

0.90 Federal Land Code 198
Exist Prob

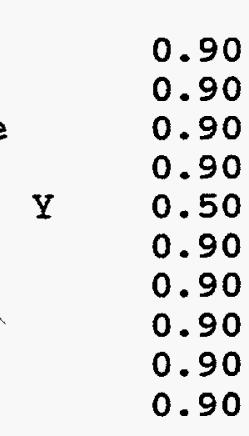


DATE: $10 / 31 / 97$

PAGE NO: 102

FERC

Number

Plant Name

stream

State

AK045 GOAT

GOAT CR

Name

AK

county Name

River Basin

WRANGELL-PETERSBURG DIV JUNEAU GROUP

Class Owner Name

NHPS/COE

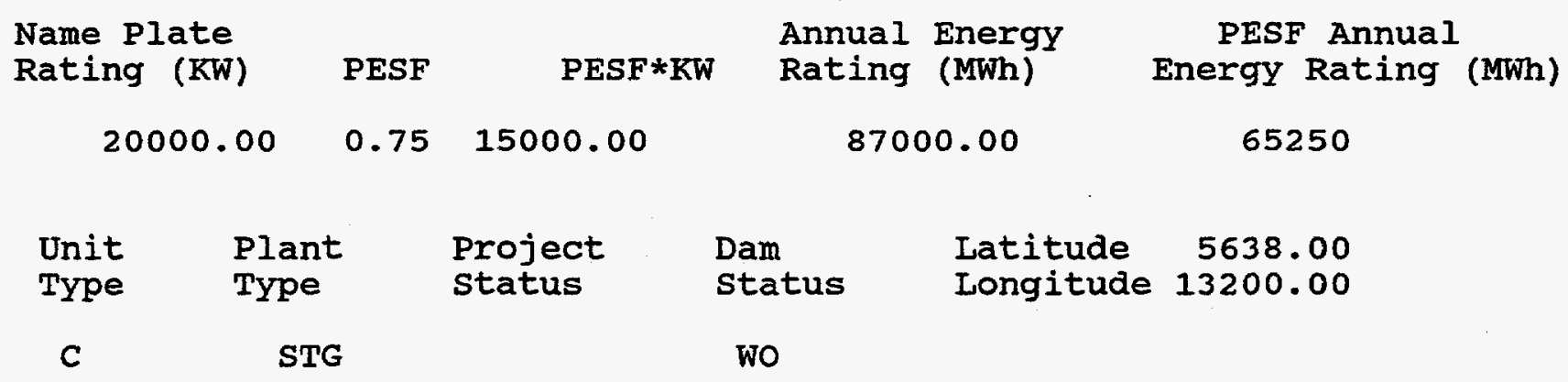

Factor

Exist Prob

Factor

Exist Prob

Wild/scenic Protection

wild/Scenic Tributary or

0.90 Wildlife value

0.90

Upstream/Downstream

Threatened/Endangered Fish

0.90

wild/Scenic Location

Threatened/Endangered Wildlife

0.90

0.90 Federal Land Code 103

0.90 Federal Land code 104

0.90

Cultural value

0.90 Federal Land Code 105

0.75

Fish Presence Value

0.90 Federal Land Code 106

Geologic Value

Historic Value

0.90 Federal Land Code 107

0.90

other value

0.90 Federal Land Code 108

0.90

Recreation Value

0.90 Federal Land Code 198

0.90

0.90

0.90

0.90 
FERC

Number

AK0 46
Plant Name

BLIND SLOUGH

County Name

WRANGELL-PETERSBURG DIV

$\begin{array}{rc}\text { Stream } & \begin{array}{c}\text { State } \\ \text { Name }\end{array} \\ \text { CRYSTAL CR,BLIND R } & \text { AK }\end{array}$

River Basin

JUNEAU GROUP $\begin{array}{cr}\text { Class } & \text { Owner Name } \\ \text { M } & \text { PETERSBURG, CITY OF }\end{array}$

Name Plate

Rating (KW)

PESF

Annual Energy

PESF*KW

Rating (MWh)

PESF Annual

1400.00

$0.50 \quad 700.00$

6800.00

3400

Unit

Plant

Type

Type

Project status

C

DIV

Factor

Exist Prob

Factor

Latitude $\quad 5636.00$

Dam

status

Longitude 13248.00

wo
Wild/Scenic Protection

wild/Scenic Tributary or Upstream/Downstream wild/Scenic Location Cultural Value

Fish Presence Value

Geologic Value

Historic Value

other Value

Recreation Value

Scenic Value

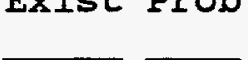

0.90

Wildife value

Threatened/Endangered Fish

Threatened/Endangered Wildlife

0.90 Federal Land Code 103

0.90 Federal Land code 104

Y $\quad 0.75$ Federal Land Code 105

0.90 Federal Land Code 106

0.90 Federal Land Code 107

$\mathrm{Y} \quad 0.75$ Federal Land Code 108

$\mathrm{Y} \quad 0.75$

0.90
Exist Prob

Y

0.75

0.90

0.90

0.90

$\mathrm{Y} \quad 0.75$

0.90

0.90

0.90

0.90

0.90 
DATE: $10 / 31 / 97$

FERC

Number

Plant Name

AK047 ANITA LAKE

county Name

WRANGELL-PETERSBURG DIV
PAGE NO: 104

State

Name

AK

\section{Class Owner Name}

NHPS/COE

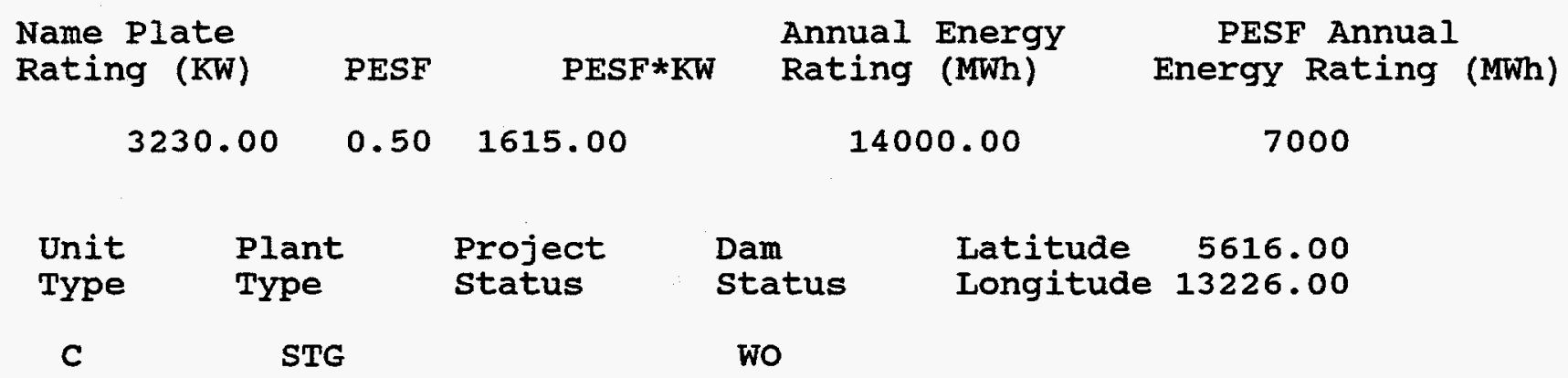

Factor

wild/Scenic Protection

wild/Scenic Tributary or

Upstream/Downstream

wild/Scenic Location

Cultural Value

Fish Presence Value

Geologic Value

Historic Value

other Value

Recreation value

Scenic Value
Exist Prob

$\begin{array}{cc} & \\ & \\ & 0.90 \\ & \\ & 0.90 \\ & 0.90 \\ Y & 0.75 \\ & 0.90 \\ & 0.90 \\ & 0.90 \\ Y & 0.75 \\ Y & 0.90\end{array}$

Factor

Exist Prob

Wildlife Value

Threatened/Endangered Fish

Threatened/Endangered Wildlife

Y

0.75

0.90

Federal Land Code 103

Federal Land Code 104

Federal Land Code 105

Federal Land Code 106

Federal Land Code 107

Federal Land Code 108

Federal Land Code 198

0.90

0.90

$\mathrm{Y} \quad 0.75$

0.90

0.90

0.90

0.90

0.90 
DATE: $10 / 31 / 97$

FERC

Number

AK0 48
Plant Name

VIRGINIA LAKE

County Name

WRANGELL-PETERSBURG DIV
PAGE NO: 105

State

Name

MILL CR

AK
Stream

\section{Class Owner Name}

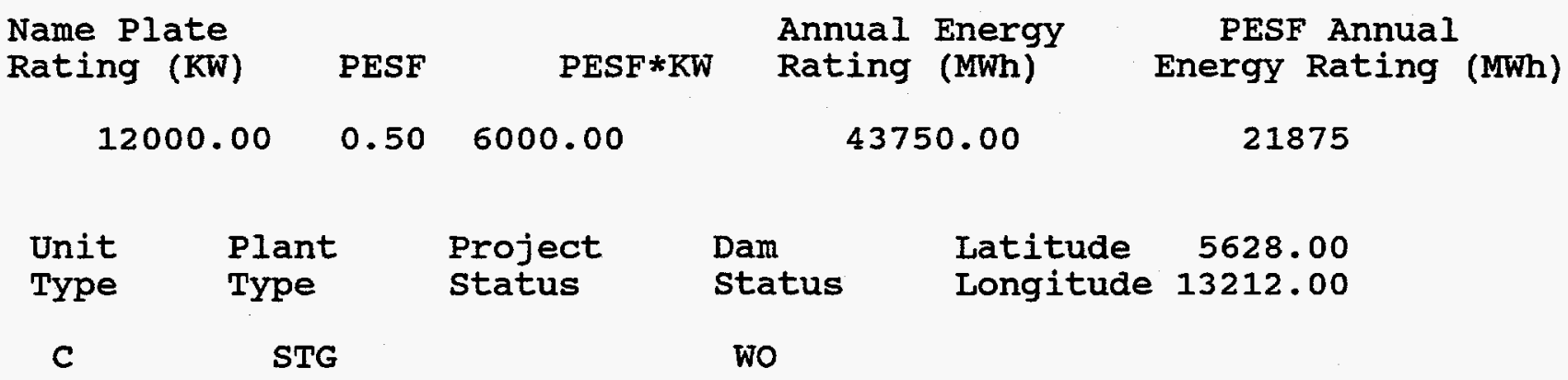

Factor

Exist Prob

wild/Scenic Protection

wild/Scenic Tributary or

Upstream/Downstream

wild/Scenic Location

Cultural value

Fish Presence Value

Geologic Value

Historic Value

other value

Recreation Value

Scenic Value
River Basin

KETCHIKAN GROUP 
DATE: $10 / 31 / 97$

PAGE NO: 106

FERC

Number

Plant Name

stream

state

Name

AK049 HARDING

HARDING $\mathbf{R}$

AK

County Name

River Basin

WRANGELL-PETERSBURG DIV

KETCHIKAN GROUP

Class

Owner Name

NHPS/COE

Name Plate

Rating (KW)

18000.00

PESF

$\mathrm{PESF} * \mathrm{KW}$

Annual Energy

Rating (MWh)

85000.00
Dam

Status

U
PESF Annual Energy Rating (MWh)

$\begin{array}{cccc}\text { Unit } & \text { Plant } & \text { Project } & \text { Dam } \\ \text { Type } & \text { Type } & \text { Status } & \text { Status } \\ \text { C } & \text { DIV } & & \text { U }\end{array}$

Factor

Exist Prob

Factor

Exist Prob

$\begin{array}{lr}\text { Latitude } & 5616.00 \\ \text { Longitude } & 13139.00\end{array}$

wild/Scenic Protection

Wild/Scenic Tributary or Upstream/Downstream wild/Scenic Location Cultural Value Fish Presence Value Geologic Value Historic Value other Value Recreation value Scenic Value

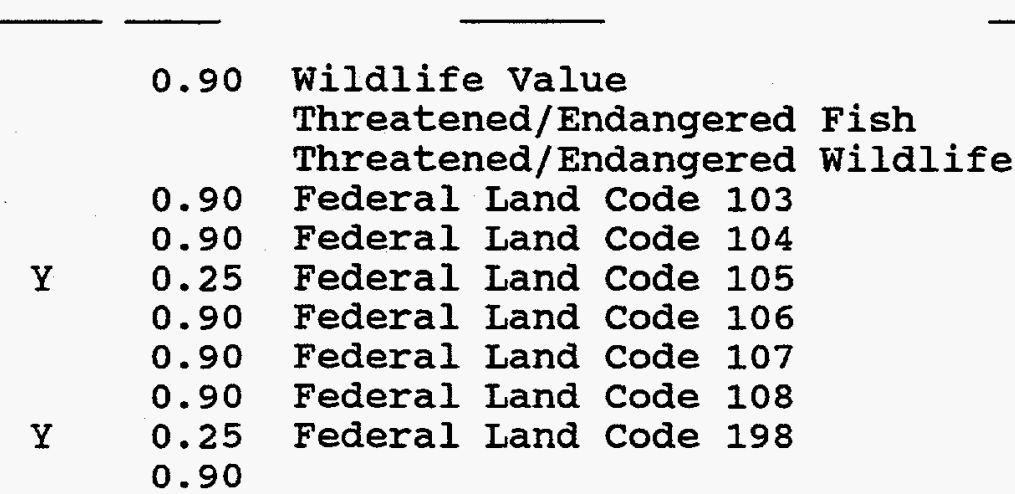

0.25

0.90

0.90

0.90

$\mathrm{Y}$

0.50

0.90

0.90

0.90

0.90

0.90 
R E S O U R C E

DATE: $10 / 31 / 97$

FERC

Number

Plant Name

AK050 LAKE GRACE

County Name

KETCHIKAN DIVISION

class
D A T A B A S E

L I S T N G

PAGE NO: 107 state

Name

AK

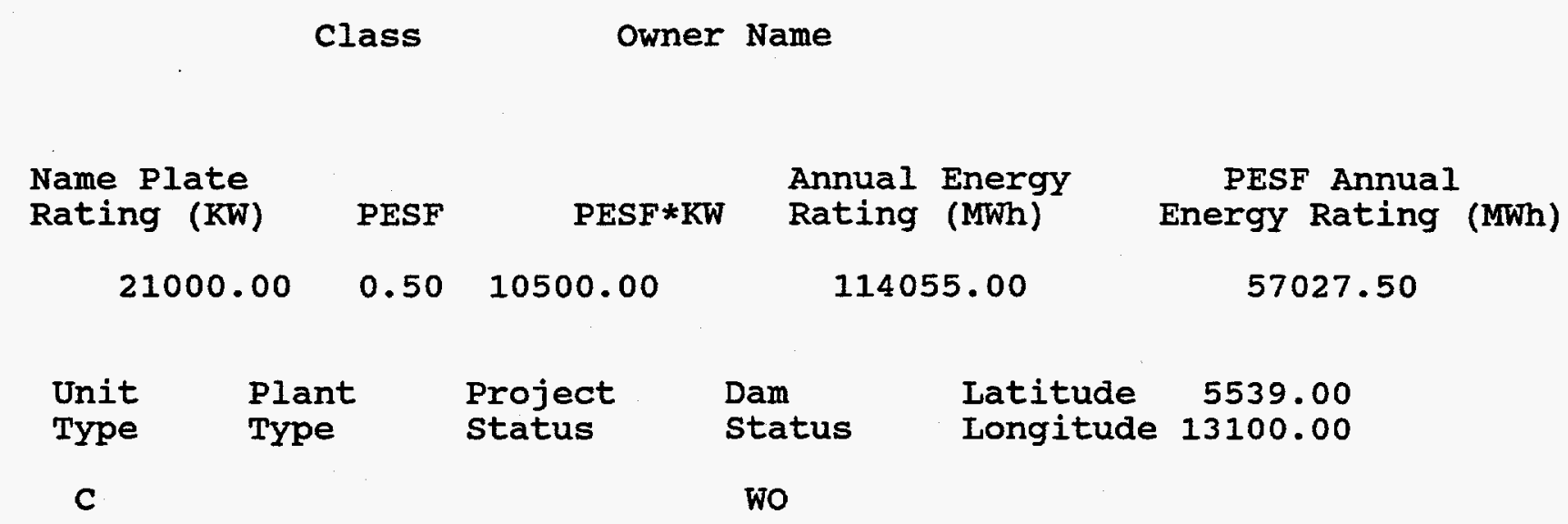

Factor

Exist Prob

Wild/Scenic Protection

Wild/Scenic Tributary or

Upstream/ Downstream

wild/Scenic Location

Cultural value

Fish Presence Value

Geologic Value

Historic Value

other Value

Recreation Value

Scenic Value
Factor

Exist Prob

\subsection{0}

Wildlife Value

Threatened/Endangered Fish

Threatened/Endangered Wildlife

0.90 Federal Land Code 103

0.90 Federal Land Code 104

$\mathrm{Y} \quad 0.75$ Federal Land Code 105

0.90 Federal Land Code 106

0.90 Federal Land Code 107

0.90 Federal Land Code 108

0.90 Federal Land Code 198

0.90
0.90

0.90

0.90

0.90

$\mathrm{Y} \quad 0.75$

0.90

Y 0.90

0.90

0.90

0.90 
DATE: $10 / 31 / 97$

PAGE NO: 108

FERC

Number

Plant Name

stream

State

Name

AK051 PURPLE LAKE

PURPLE LAKE OUTLET

AK

county Name

OUTER KETCHIKAN DIV
River Basin

KETCHIKAN GROUP $\begin{array}{cc}\text { Class } & \text { Owner Name } \\ \text { M } & \text { METLAKATLA PWR AND LIGHT }\end{array}$

Name Plate Rating (KW) 500.00

PESF

$0.75 \quad 375.00$
Annual Energy Rating (MWh)

2770.00
PESF Annual Energy Rating (MWh)

$\begin{array}{cccllr}\text { Unit } & \text { Plant } & \text { Project } & \text { Dam } & \text { Latitude } & 5504.00 \\ \text { Type } & \text { Type } & \text { Status } & \text { Status } & \text { Longitude } 13116.00 \\ \text { C } & \text { STG } & \text { LJ } & W & \end{array}$

Factor

Wild/Scenic Protection

wild/Scenic Tributary or Upstream/Downstream wild/Scenic Location Cultural value Fish Presence Value Geologic Value Historic Value other Value Recreation value Scenic value
Exist Prob

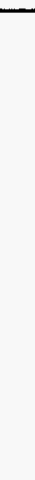

Exist Prob

Factor

$$
2077.50
$$


$\begin{array}{llllllll}R & E & S & O & U & R & \text { C }\end{array}$

DATE: $10 / 31 / 97$

FERC

Number

Plant Name

AK052

MAHONEY LAKE

County Name

KETCHIKAN DIVISION

class
D A T A B A S E

\begin{abstract}
D
\end{abstract}
L I S T I N G

PAGE NO: 109

$\begin{array}{cc}\text { Stream } & \begin{array}{c}\text { State } \\ \text { Name }\end{array} \\ \text { MAHONEY CR } & \text { AK } \\ \text { River Basin } & \\ \text { KETCHIKAN GROUP } & \end{array}$

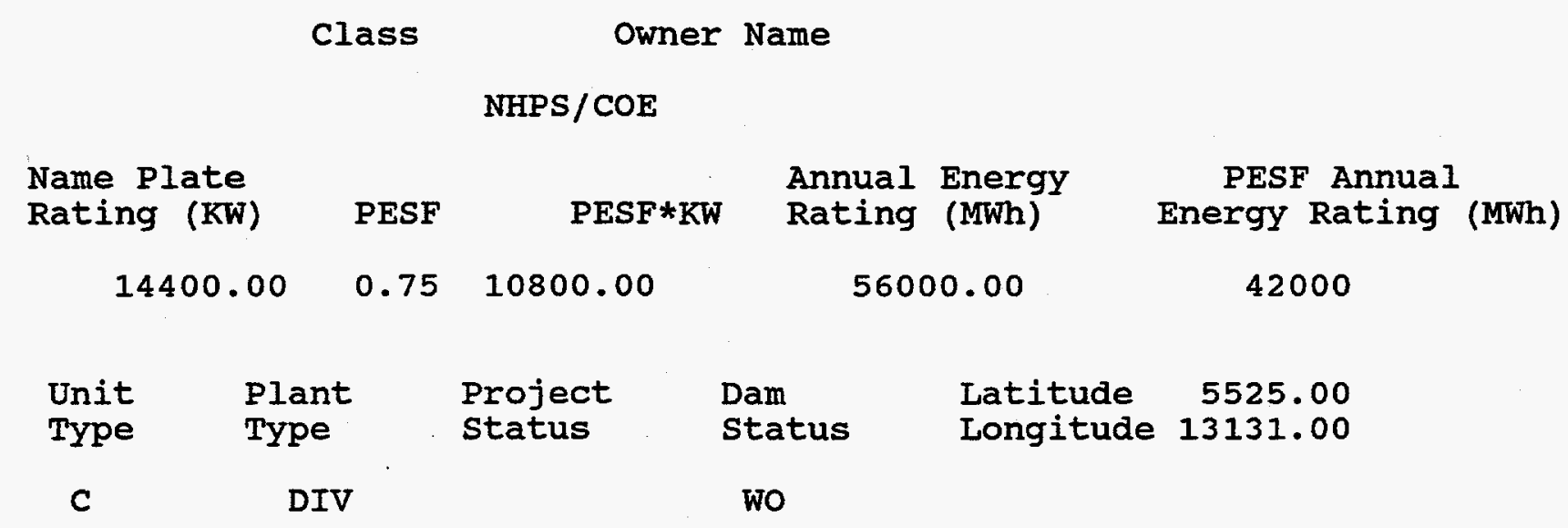

Factor

Wild/Scenic Protection

wild/scenic Tributary or Upstream/Downstream wild/Scenic Location Cultural value Fish Presence Value Geologic Value Historic Value other Value

Recreation Value

Scenic Value
Exist Prob

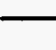

0.90

Factor

Exist Prob

Wildlife Value

Threatened/Endangered Fish Threatened/Endangered Wildlife Federal Land Code 103 Federal Land Code 104 Federal Land Code 105 Federal Land Code 106 Federal Land Code 107 Federal Land Code 108 Federal Land Code 198 
FERC

Number

AK053 UPPER SILVIS

County Name

KETCHIKAN DIVISION
Stream

BEAVER FALLS CR, GEORGE INLET
State

Name

AK

Class Owner Name

M KETCHIKAN, CITY OF

Name Plate Rating ( $K W$ )

20000.00
PESF

$0.50 \quad 10000.00$

Unit

Type

$$
\begin{aligned}
& \text { Plant } \\
& \text { Type } \\
& \text { STG }
\end{aligned}
$$

Factor

Exist Prob

Wild/Scenic Protection

wild/Scenic Tributary or

Upstream/Downstream

wild/Scenic Location

Cultural Value

Fish Presence Value

Geologic Value

Historic Value

other Value

Recreation value

Scenic Value
Annual Energy

Rating (MWh)

49110.00
PESF Annual Energy Rating (MWh)

24555 $\begin{array}{llr}\text { Dam } & \text { Latitude } & 5523.00 \\ \text { Status } & \text { Longitude } & 13131.00\end{array}$

$\begin{array}{llr}\text { Dam } & \text { Latitude } & 5523.00 \\ \text { Status } & \text { Longitude } & 13131.00\end{array}$

wo

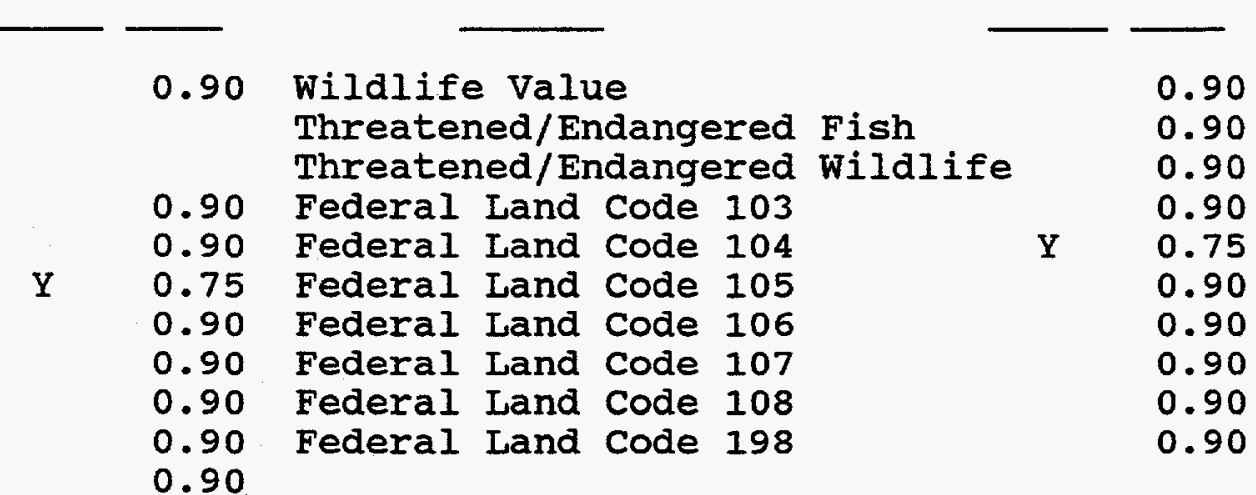


R E S O U R C E

DATE: $10 / 31 / 97$

FERC

Number

Plant Name

AK054 KETCHIKAN LAKES

County Name

KETCHIKAN DIVISION

Class Owner Name

M KETCHIKAN, CITY OF

Name Plate

Rating (KW)

$$
2000.00
$$

PESF

PESF*KW

Annual Energy

Rating (MWh)

15000.00

$0.50 \quad 1000.00$

Unit

Plant

Type

Type

Project status

C

DIV

Factor

Exist Prob

Latitude

Longitude 13137.00

Dam

Status

wo
PAGE NO: 111

State

Name

AK
PESF Annual Energy Rating (MWh)

7500

Wild/Scenic Protection

wild/Scenic Tributary or

Upstream/Downstream

wild/Scenic Location

cultural value

Fish Presence Value

Geologic Value

Historic Value

other Value

Recreation Value

Scenic Value

Factor

\subsection{0}

Wildlife value

Threatened/Endangered Fish

Threatened/Endangered Wildlife

0.90 Federal Land Code 103

0.90 Federal Land code 104

$Y \quad 0.75$ Federal Land Code 105

0.90 Federal Land Code 106

$\mathrm{Y} \quad 0.75$

0.90

$\mathrm{Y} \quad 0.75$ Federal Land Code 108

Federal Land Code 198
Exist Prob

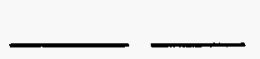

0.90

0.90

0.90

0.90

0.90

0.90

0.90

0.90

0.90

0.90

0.90 
FERC

Number

Plant Name

AK055 LAKE CONNELL

County Name

KETCHIKAN DIVISION
Stream

WARD CR, WARD COVE

River Basin

KETCHIKAN GROUP

\section{Class Owner Name}

I KETCHIKAN PULP CO

Name Plate Rating (KW) PESF

Annual Energy 2000.00 0.10200 .00 PESF*KW Rating (MWh)

10400.00

Dam status

Latitude Longitude 13140.00 state Name

AK

$\begin{array}{cclll}\text { Unit } & \text { Plant } & \text { Project } & \text { Dam } & \text { Latitude } 5526.00 \\ \text { Type } & \text { Type } & \text { Status } & \text { Status } & \text { Longitude 13140.00 } \\ \text { C } & \text { DIV } & & \text { U }\end{array}$

Factor

Exist Prob

wild/Scenic Protection

wild/Scenic Tributary or Upstream/Downstream wild/Scenic Location Cultural Value Fish Presence Value Geologic Value Historic Value other value Recreation Value Scenic Value
Factor

Exist Prob

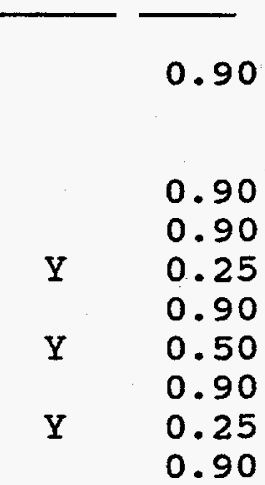

wildife Value Threatened/Endangered Fish Threatened/Endangered Wildlife Federal Land Code 103 Federal Land Code 104 Federal Land Code 105 Federal Land Code 106 Federal Land Code 107 Federal Land Code 108 Federal Land Code 198
PESF Annual Energy Rating (MWh) 1040 
R E S O U R C E

DATE: $10 / 31 / 97$
D A T A B A S E

I I S I N G

PAGE NO: 113

FERC

Number

Plant Name

AK056 LINKUM

county Name

PRINCE OF WALES DIV stream

LINKUM CR, KASAAN BAY

River Basin

KETCHIKAN GROUP

$\begin{array}{cc}\text { Class } & \text { Owner Name } \\ \text { I } & \text { PACIFIC AMERICAN FISHERIES }\end{array}$

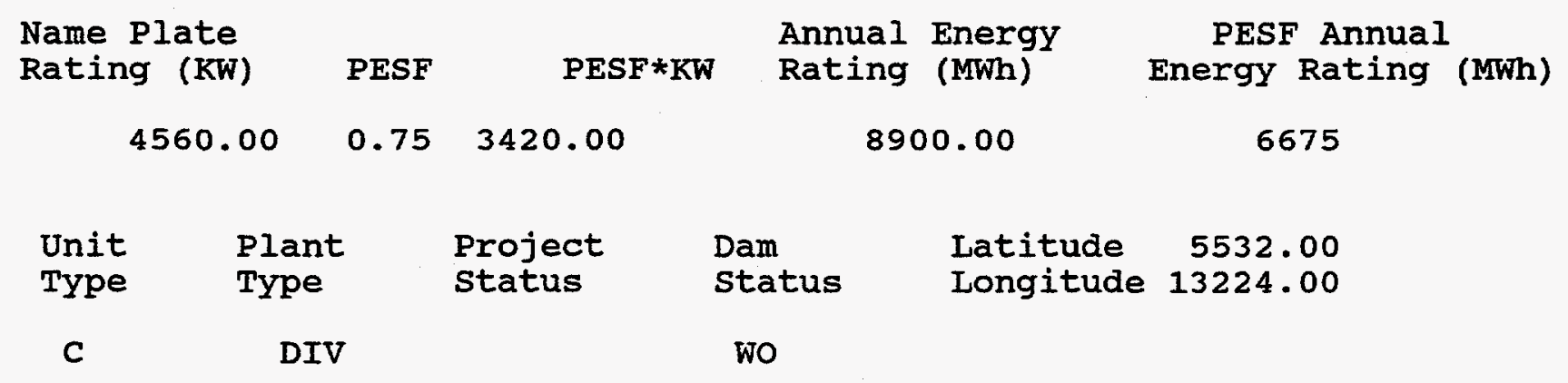

Factor

Wild/Scenic Protection

wild/Scenic Tributary or

Upstream/Downstream

wild/Scenic Location

Cultural Value

Fish Presence Value

Geologic Value

Historic Value

other value

Recreation value

Scenic value
Exist Prob

Factor

Exist Prob
State

Name

AK 
DATE: $10 / 31 / 97$

PAGE NO: 114

FERC

Number

Plant Name

Stream

State Name

AK057 LAKE MARY

OLD FRANKS CR

AK

county Name

River Basin

PRINCE OF WALES DIV

KETCHIKAN GROUP

Class Owner Name

NHPS/COE

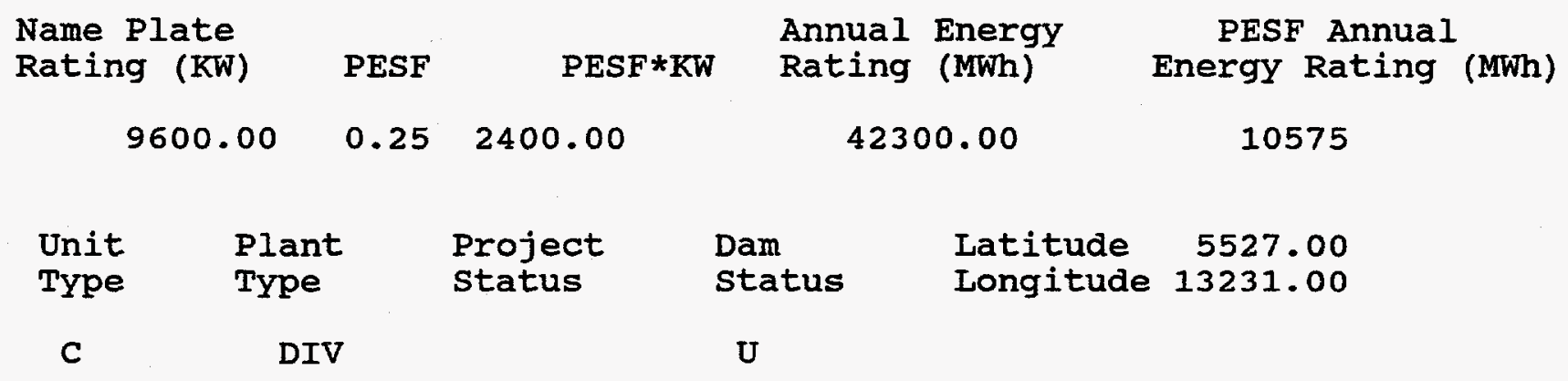

Factor

Exist Prob

Factor

Exist Prob

Wild/Scenic Protection

Wild/Scenic Tributary or

Upstream/Downstream

wild/Scenic Location Cultural value

Fish Presence Value

Geologic Value

Historic Value

other value

Recreation value

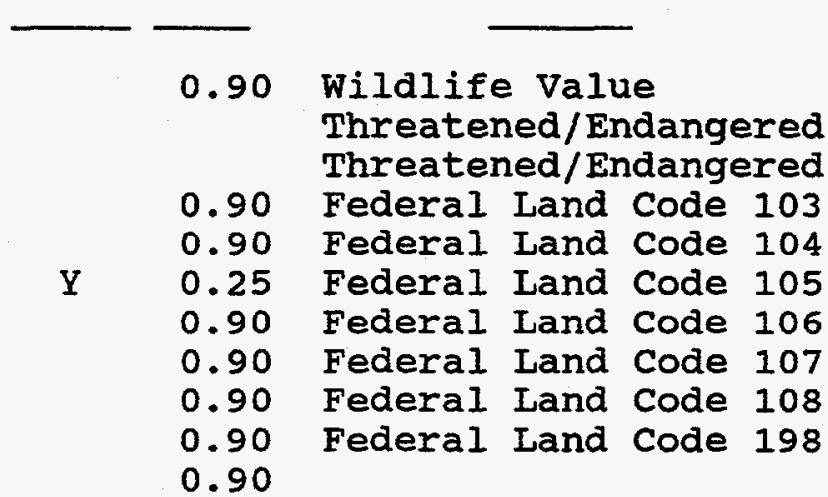

Exist Prob

Scenic Value

0.90

0.90

0.90

0.90

0.90

0.90

0.90

0.90

0.90

0.90

0.90 
DATE: $10 / 31 / 97$

PAGE NO: 115

FERC

Number

AK058
Plant Name

BLACK BEAR

County Name

PRINCE OF WALES DIV

Class Owner Name

NHPS/COE

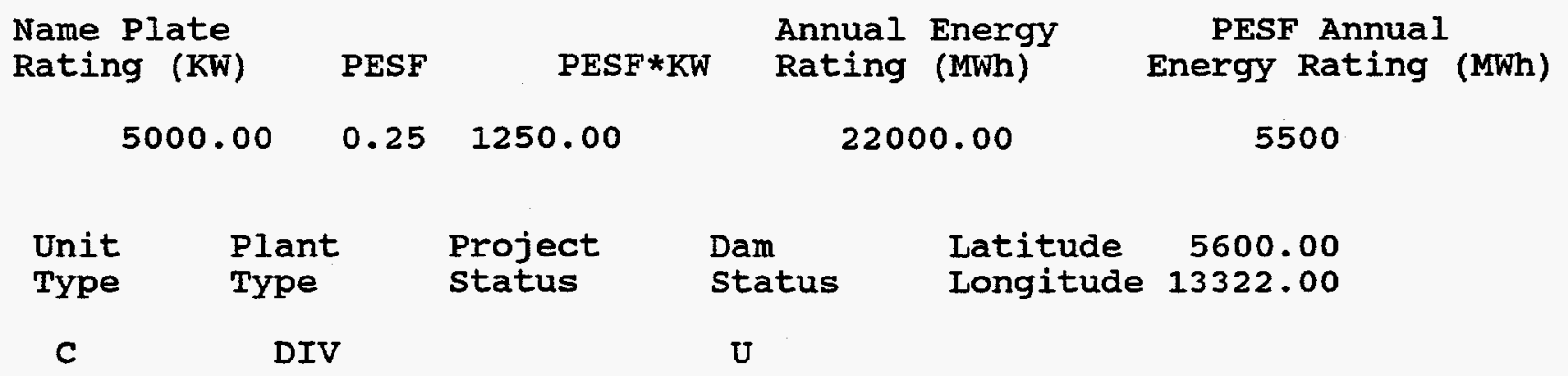

Factor

Exist Prob

Wild/Scenic Protection

Wild/Scenic Tributary or Upstream/Downstream wild/Scenic Location Cultural value Fish Presence Value

Geologic Value Historic Value other Value

Recreation Value Scenic Value
Factor

Exist Prob

0.90


0.90
0.90
0.25
0.90
0.90
0.90
0.90
0.90

state

Name

AK
River Basin

KETCHIKAN GROUP
BLACK BEAR CR

stream

P


FERC

Number

AK059 SCAMMON BAY

County Name

WADE HAMPTON DIV
Plant Name

Class

Owner Name

F CORPS OF ENGINEERS

Name Plate

Rating (KW)

PESF

Annual Energy

PESF*KW

Rating (MWh)

PESF Annual

100.00

$0.90 \quad 90.00$

372.00

334.80

\section{Project status}

FA

$\begin{array}{llr}\text { Dam } & \text { Latitude } & 6150.00 \\ \text { Status } & \text { Longitude } & 16527.00\end{array}$

$\mathrm{U}$
State

Name

AK
C

ROR
Exist Prob

Wild/Scenic Protection

Wild/Scenic Tributary or

Upstream/Downstream

wild/Scenic Location

Cultural value

Fish Presence Value

Geologic Value

Historic Value

other Value

Recreation Value

Scenic Value

0.90
Factor

Exist Prob

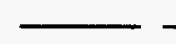

\subsection{0}

Wildlife Value

Threatened/Endangered Fish

0.90 Federal Land Code 103

0.90 Federal Land Code 104

0.90 Federal Land Code 105

0.90 Federal Land Code 106

0.90 Federal Land Code 107

0.90 Federal Land Code 108

0.90 Federal Land code 198
0.90

0.90

0.90

0.90

0.90

0.90

0.90

0.90

0.90

0.90 
R E S O U R C E

DATE: $10 / 31 / 97$

FERC

Number

Plant Name

AK060 PYRAMID CREEK

County Name

ALEUTIAN ISLANDS DIV
D A T A B A S E

L I S T N G

PAGE NO: 117

$\begin{array}{cc}\text { Stream } & \begin{array}{c}\text { state } \\ \text { Name }\end{array} \\ \text { PYRAMID CR, PACIFIC OCEAN } & \text { AK } \\ \text { River Basin } & \\ \text { ALASKA PENINSULA RIVER BAS } & \end{array}$

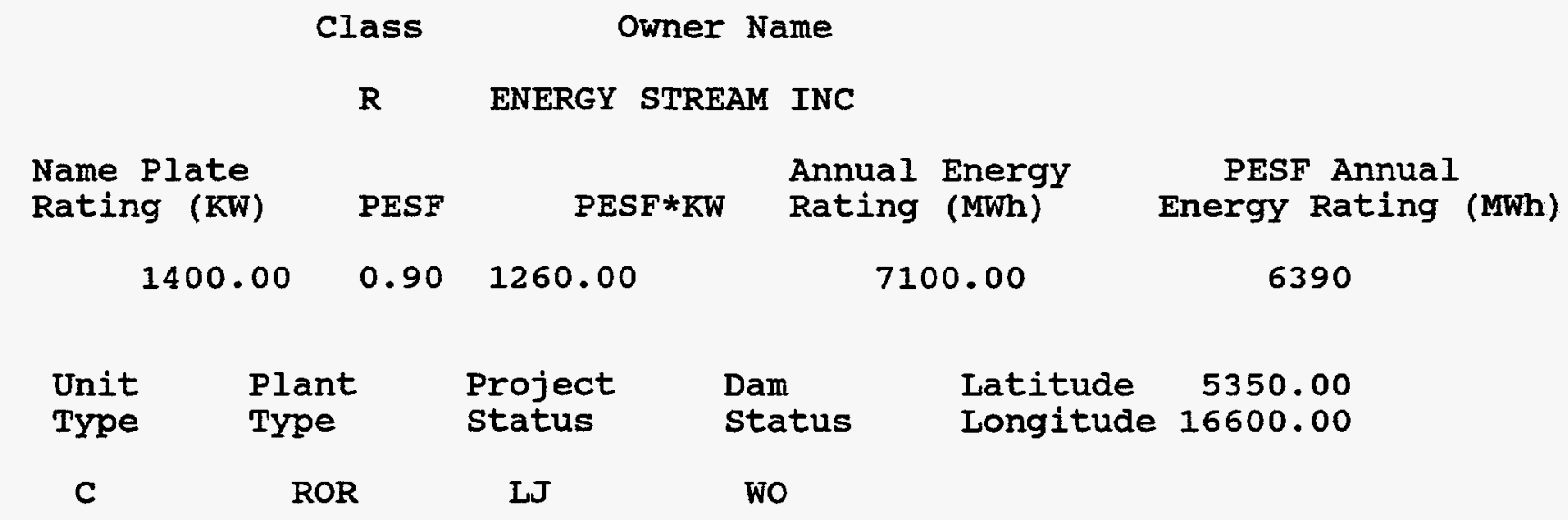

Factor

Wild/Scenic Protection

wild/Scenic Tributary or Upstream/Downstream wild/Scenic Location Cultural Value Fish Presence Value

Geologic Value Historic Value other value

Recreation Value Scenic Value
Exist Prob

Factor

Exist Prob

$\begin{array}{ll}0.90 & \text { Wildlife Value } \\ & \text { Threatened/Endangered Fish } \\ & \text { Threatened/Endangered Wildlife } \\ 0.90 & \text { Federal Land Code } 103 \\ 0.90 & \text { Federal Land Code } 104 \\ 0.90 & \text { Federal Land Code } 105 \\ 0.90 & \text { Federal Land Code } 106 \\ 0.90 & \text { Federal Land Code } 107 \\ 0.90 & \text { Federal Land Code } 108 \\ 0.90 & \text { Federal Land Code } 198 \\ 0.90 & \end{array}$

0.90

0.90

0.90

0.90

0.90

0.90

0.90

0.90

0.90 


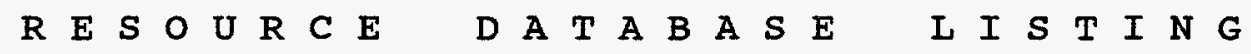

DATE: $10 / 31 / 97$

PAGE NO: 118

FERC

Number

Plant Name

Stream

state

AK061 SOUTH FORK EAGLE RIVER

S FK EAGLE R

Name

AK

county Name

ANCHORAGE DIVISION
River Basin

KENAI PENINSULA RIVER BAS
Class

$\mathbf{R}$

Name Plate

Rating ( $\mathrm{KW})$

1100.00
PESF

0.75

825.00

Project status

Unit
Type

Plant

Type

ROR

Factor

Wild/Scenic Protection

Wild/Scenic Tributary or Upstream/Downstream wild/Scenic Location Cultural value

Fish Presence Value

Geologic Value

Historic Value

other Value

Recreation Value

Scenic Value
Exist Prob

0.90

Wildife value

Threatened/Endangered Fish

Threatened/Endangered Wildlife

0.90 Federal Land Code 103

0.90 Federal Land Code 104

Y $\quad 0.75$ Federal Land Code 105

0.90 Federal Land Code 106

0.90 Federal Land Code 107

0.90 Federal Land Code 108

0.90 Federal Land Code 198
PESF Annual Energy Rating (MWh)

2625

0.90

Exist Prob

0.90

0.90

0.90

0.90

0.90

0.90

0.90

0.90

0.90

0.90 
FERC

Number

Plant Name

AK062 PALMER

County Name

ANCHORAGE DIVISION

\section{Stream}

MCROBERTS CREEK

River Basin

KENAI PENINSULA RIVER BAS
State

Name

AK

\section{Class Owner Name \\ R AUSMAN AND ELLIS}

Name Plate

Rating (KW)

400.00

PESF

Annual Energy

PESF*KW Rating (MWh)

1700.00

0.25100 .00

Project

status

$\begin{array}{cc}\text { Type } & \begin{array}{c}\text { Plant } \\ \text { Type }\end{array} \\ \text { C } & \text { ROR }\end{array}$

Factor

$\begin{array}{llr}\text { Dam } & \text { Latitude } & 6033.00 \\ \text { Status } & \text { Longitude } & 14906.00\end{array}$

U
PESF Annual Energy Rating (MWh)

425
Wild/Scenic Protection

wild/Scenic Tributary or

Upstream/Downstream

wild/Scenic Location

Cultural Value

Fish Presence Value

Geologic Value

Historic Value

other Value

Recreation Value

Scenic Value
Exist Prob

Factor

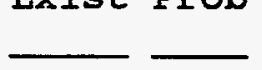

0.90

wildife Value Threatened/Endangered Fish Threatened/Endangered Wildlife

Exist Prob

0.90







0.90
0.90
0.25
0.90
0.90
0.90
0.90

Federal Iand Code 104

Federal Land Code 105

Federal Land Code 106

Federal Land Code 107

Federal Land code 108

Federal Land Code 198

Exist Prob

0.90

0.90

0.90

0.90

0.90

0.90

0.90

0.90

0.90

0.90 